\title{
Correlation-based
}

\section{seismic velocity inversion}

Tristan van Leeuwen Delft University of Technology 



\title{
Correlation-based seismic velocity inversion
}

\author{
Proefschrift \\ ter verkrijging van de graad van doctor \\ aan de Technische Universiteit Delft, \\ op gezag van de Rector Magnificus Prof.ir. K.C.A.M. Luyben, \\ voorzitter van het College voor Promoties, \\ in het openbaar te verdedigen, \\ op maandag 23 augustus 2010 om 12:30 uur \\ door \\ Tristan VAN LEEUWEN \\ doctorandus in de Computational Science \\ geboren te Hilversum
}


Dit proefschrift is goedgekeurd door de promotor:

Prof.dr. W.A. Mulder

Samenstelling promotiecommissie:

Rector Magnificus

Prof.dr. W.A. Mulder

Prof.dr.ir. C.P.A. Wapenaar

Prof.dr.ir. R. Arts

Prof.dr. J. Trampert

Prof.dr.ir. C.W. Oosterlee

Prof.dr. K.J. Batenburg

Dr. A.P.E. ten Kroode

\section{Voorzitter}

Technische Universiteit Delft, Promotor

Technische Universiteit Delft

Technische Universiteit Delft

Universiteit Utrecht

Technische Universiteit Delft

Universiteit Antwerpen

Shell International Exploration and Production

ISBN-978-90-8891-186-6

Copyright by Tristan van Leeuwen 2010. Some Rights Reserved. This work is licensed under a Creative Commons Attribution 3.0 Unported License. Chapters 3 and 5 are adapted from published work (DOIs:10.1111/j.1365-2478.2008.00704.x, 10.1111/j.1365-246X.2010.04681.x) and reproduced here with permission from John Wiley and Sons and the Royal Astronomical Society. Chapter 4 is adapted from published work (DOI:10.1088/0266-5611/26/1/015008) and reproduced here with permission from IOP Publishing. The fotograph on the backcover is copyrighted by Sam Rentmeester/FMAX.

Published by: Uitgeverij BOXPress, Oisterwijk, The Netherlands Printed by: Proefschriftmaken.nl | Printyourthesis.com 


\section{Contents}

1 Introduction 1

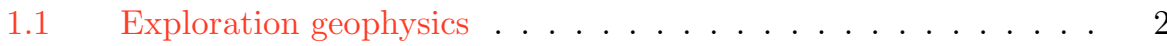

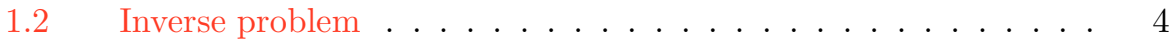

1.3 This thesis ......................... 5

$2 \quad$ Modelling and inversion of seismic data $\quad 7$

2.1 Modelling of seismic data in the acoustic approximation . . . . 8

2.1.1 Linearization . . . . . . . . . . . . . 10

2.1.2 Asymptotic solution of the wave equation . . . . 11

2.2 Sensitivity analysis . . . . . . . . . . . . . . 13

2.2.1 Amplitude sensitivity via the Born approximation . 13

2.2.2 Phase sensitivity via the Rytov approximation . . . 14

2.2.3 Uniqueness . . . . . . . . . . . . . . . . . 17

2.3 Inversion of seismic data . . . . . . . . . . . . . . . . . 17

2.3.1 Full waveform inversion . . . . . . . . . . 17

2.3.2 Linearized inversion .............. . . . . . 18

2.3.3 Migration Velocity Analysis . . . . . . . . . . 20

2.3.4 Reflection tomography . . . . . . . . . 22

2.3.5 Transmission tomography . . . . . . . . . . 23

2.4 Summary ....................... 24

$3 \quad$ Velocity analysis based on data correlation $\quad \mathbf{2 5}$

3.1 Introduction . . . . . . . . . . . . . . 26

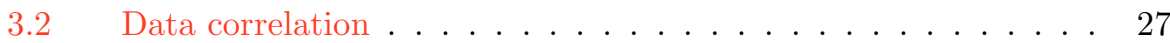

3.3 Generalized imaging conditions . . . . . . . . . . . . 31

3.4 Similarities between data and image-domain focusing . . . . . . 33

3.5 Tests with synthetic data . . . . . . . . . . . . . 34

3.6 Test with real data . . . . . . . . . . . . . . . . . . . . . . . . . . . . . 37

3.7 Conclusions and discussion . . . . . . . . . . . . . . . . . . . . . . . . . . . . 39

3.8 Appendix: Gradient . . . . . . . . . . . . . . . . 40

4 A comparison of seismic velocity analysis methods 43

4.1 Introduction . . . . . . . . . . . . . . . . . . 44

4.2 Notation and preliminaries . . . . . . . . . . . . 45 
4.3 Modelling ..................... 46

4.3.1 Laterally invariant velocity . . . . . . . . . . 47

4.4 Linearized inversion . . . . . . . . . . . . . . . . . . 49

4.5 Velocity analysis in the image domain . . . . . . . . . . . . . 50

4.5.1 Stackpower . . . . . . . . . . 50

4.5.2 Differential semblance ............. 51

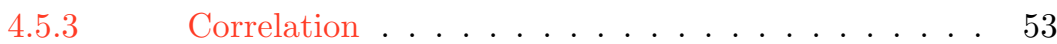

4.6 Data domain velocity analysis . . . . . . . . . . . . . 57

4.6.1 Least-squares . . . . . . . . . . . . . . 58

4.6.2 Correlation .................. 59

4.7 Focusing in the image and data domain . . . . . . . . . . . . . 62

4.8 Synthetic-data example . . . . . . . . . . . . 63

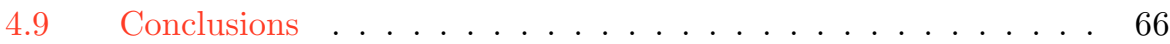

$5 \quad$ Correlation-based wave-equation traveltime tomography. $\quad \mathbf{6 7}$

5.1 Introduction . . . . . . . . . . . . . . 68

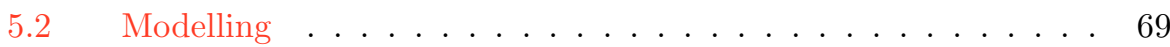

5.3 Correlation-based criteria . . . . . . . . . . . . . 70

5.3.1 Picking approach . . . . . . . . . . . 71

5.3.2 Weighted norm approach ........... . . 71

5.4 Sensitivity kernels . . . . . . . . . . . . . . 76

5.5 Example: diving wave tomography . . . . . . . . . . . . 79

5.6 Application: cross-well tomography . . . . . . . . . . . . 80

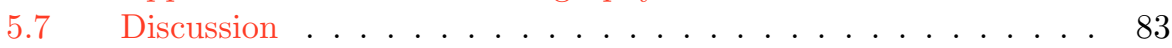

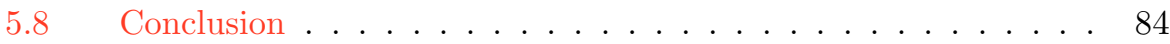

5.9 Appendix A: Weighted norm of the correlation . . . . . . . . 84

5.10 Appendix B: Sensitivity kernels . . . . . . . . . . . 86

$6 \quad$ Correlation-based wave-equation reflection tomography $\quad \mathbf{8 7}$

6.1 Introduction . . . . . . . . . . . . . . . . 88

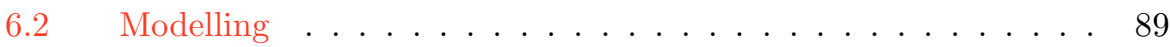

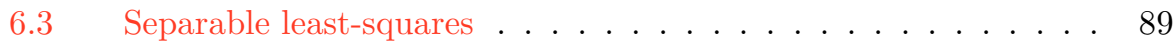

6.4 Reflection tomography ................. 90

6.4.1 Projection.................... 91

6.4.2 Correlation: detection of traveltime shifts . . . . . 92

6.5 Sensitivity Kernel . . . . . . . . . . . . . . . . . . . . 92

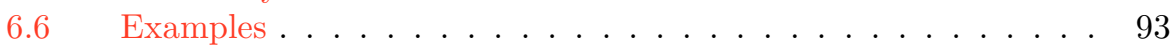

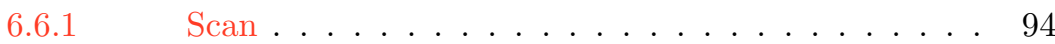

6.6.2 Gradient ................. 96

6.6 .3 Inversion ............... 96

6.7 Conclusion \& discussion . . . . . . . . . . . . . . . . . . 97

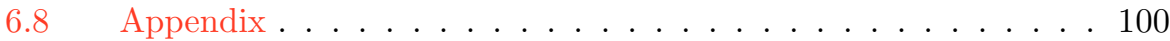

6.8.1 $\delta \mathcal{F}\left[c_{0}\right] \ldots \ldots \ldots \ldots \ldots \ldots$

6.8.2 $\delta \mathcal{F}^{*}\left[c_{0}\right] \ldots \ldots \ldots \ldots \ldots \ldots \ldots$

6.8.3 $\partial_{c}\left(\pi\left[c_{0}\right] \bar{d}\right) \ldots \ldots \ldots 10 \ldots \ldots \ldots$ 
7.1 Extension of the projection operator . . . . . . . . . . 105

7.2 Approximate projection . . . . . . . . . . . 105

7.3 Correlation-based misfit . . . . . . . . . . . . . 105

7.4 Computational efficiency . . . . . . . . . . . . 105

7.5 Tomography vs. waveform inversion . . . . . . . . . 105

$\begin{array}{ll}\text { Bibliography } & 107\end{array}$

$\begin{array}{ll}\text { Summary } & 114\end{array}$

$\begin{array}{ll}\text { Samenvatting } & 115\end{array}$

$\begin{array}{ll}\text { Curriculum Vitae } & 116\end{array}$

$\begin{array}{ll}\text { Acknowledgments } & 117\end{array}$ 


\section{1 Introduction: The seismic inverse problem}




\subsection{Exploration geophysics}

Since humanity started using oil on an industrial scale, exploration geophysicists have been looking for better ways to detect and extract hydrocarbons from the subsurface. And, seen that hydrocarbons are still our main source of energy, this effort is expected to continue in the foreseeable future.

In the early days, geologists studied rock formations above ground. Oil often gets trapped underneath domed structures and their presence can be readily inferred on the surface - if they are not too deep. Also, the presence of certain minerals, in a water well for example, could indicate oil. Sometimes, people would resort to doodlebugging - using a divining rod to locate oil. In fact, the term 'doodlebugger' still refers to a person that is looking for oil, albeit with far more sophisticated methods.

During the first world war, seismographs were being used to locate heavy artillery and it was soon realized that this technique could be employed to detect subsurface structures. This is the birth of the seismic experiment and the basic principle has not changed since. The typical setup of such an experiment is depicted in figure 1.1. Sources and receivers are placed at, or just below, the surface and the responses of the sequentially detonated sources are recorded by the receivers for a certain amount of time. Typically, hundreds of sources and thousands of receivers are spread out on an area of a few square kilometres with a spacing of tens of meters. For each source, typically 10 seconds are recorded. With these data one can probe the subsurface below the sources up to $5-10 \mathrm{~km}$ depth with a resolution of roughly 50 meters. Although gravity and electromagnetic prospecting methods are also available, the seismic experiment is by far the most widely used in the search for oil.

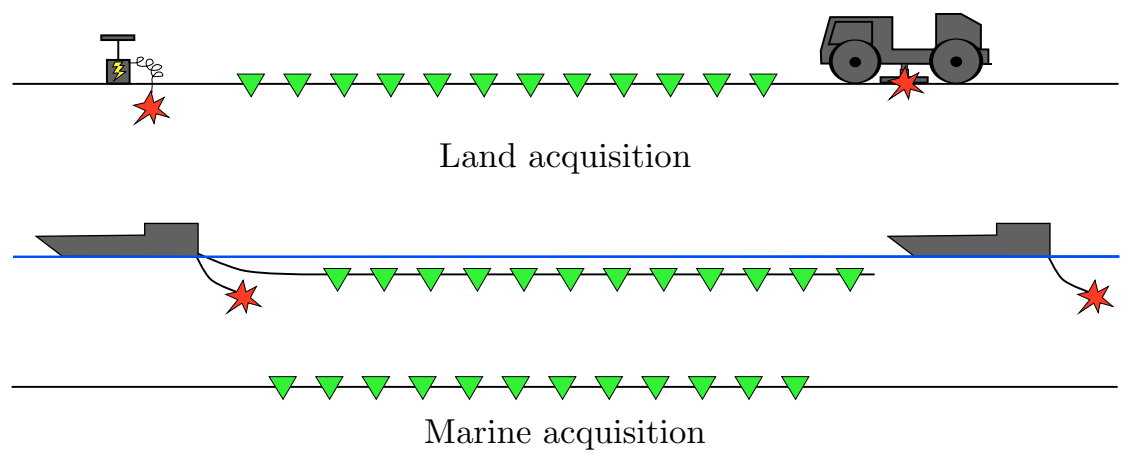

Figure 1.1. Typical seismic experiment. Typical seismic sources on land are either dynamite or a vibrating plate, usually mounted on a truck. Geophones are placed in straight lines. For a marine survey, the source is usually an airgun suspended from a boat. The receivers are hydrophones which can be either towed behind a vessel or placed on the ocean bottom. In both cases the experiment is repeated for several different source positions with possibly varying receiver layouts. 
The recorded seismic data contain mainly reflections, caused by rapid variations in the medium properties. The short-offset data can be seen as an echogram of the earth, and can be interpreted as such. Figure 1.2 gives an example. Such images were the basis of seismic exploration in the early days. But, exploration is moving towards finding smaller reservoirs in geologically more complex areas where the echoes cannot be interpreted directly anymore. As a result, geophysicists are using physical knowledge of wave propagation to map the data from time back to depth. Using a geometric optics approximation to wave propagation, events in the data can be traced back along the geometric ray path to the reflector whence they came. This is the basis of what geophysicists call migration. While migration was being done manually with ruler and pencil in the early days, today advanced computational algorithms are being used. Of course, to be able to migrate the data, the speed with which the waves travel needs to be known. The inference of the subsurface velocity is called velocity analysis. Again, techniques have been developed to (partly) automate this process. Finally, elastic parameters can be obtained by studying the amplitudes of the data originating from a certain reflector. Such a process is referred to as inversion in the geophysical literature. In this thesis we will use the term inversion in a broader sense, as will be explained in the next section. These three steps - velocity analysis, migration, inversion - are still the basis of seismic processing workflows in use today. Yet, even with all the sophisticated techniques, the transformation of raw seismic data into a useable image still requires a lot of manual labour from highly trained staff. This drives the quest for ever better, automated techniques and algorithms.

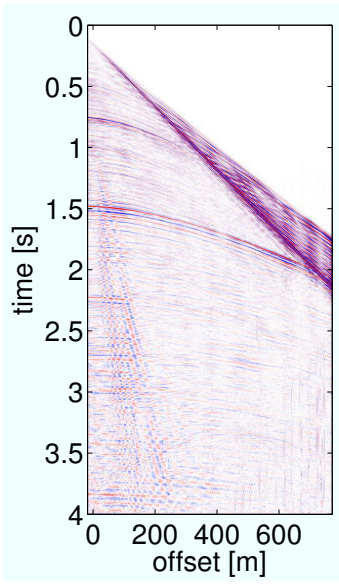

Shotgather

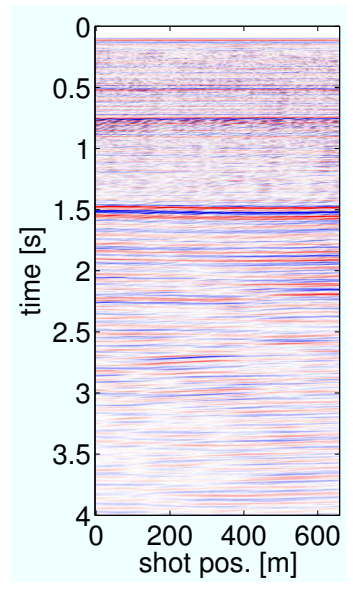

Stack

Figure 1.2. Typical marine seismic data. The shotgather depicts the response of a single airgun burst (shot). The stack is formed by summing the offsets for each shot. 


\subsection{Inverse problem}

In the early 1980's the availability of digital computers and advances in the field of inverse problems culminated in the formulation of an automated procedure that promised to be able to infer a complete quantitative image of the subsurface from seismic data, basically combining velocity analysis, migration and inversion. This formulation is based on a finite-frequency description of wave propagation as a function of the different elastic parameters, here denoted by $\nu$. We can then say that the data, as measured in the field, are the result of an operator, $\mathcal{F}$ acting on the model parameters:

$$
d=\mathcal{F}[\nu]
$$

We measured $d$ and we have access to $\mathcal{F}$ - via computer simulation, for example so we should be able to find a $\nu$ such that above equation holds. The problem of finding such a $\nu$ is called an inverse problem. Evaluation of $\mathcal{F}[\nu]$ is the corresponding forward problem.

The French mathematician Jacques Hadamard used the following criteria to define the well-posedness of an inverse problem:

- Existence: For given data, $d$, there exists a $\nu$ such that $\mathcal{F}[\nu]=d$.

- Uniqueness: There is exactly one such $\nu$.

- Stability: A small perturbation in the data causes a small perturbation in the solution.

When the inverse problem fails to satisfy one of the above conditions it is called an ill-posed problem. As opposed to forward problems, inverse problems are usually ill-posed.

To circumvent the existence issue, the inverse problem can be cast as a non-linear least-squares optimisation problem:

$$
\nu^{*}=\underset{\nu}{\operatorname{argmin}}\|\mathcal{F}[\nu]-d\|_{2}^{2}
$$

To address the stability and uniqueness, regularisation techniques have been developed. The Russian mathematician Andrey Tikhonov was the first to develop a comprehensive theory behind the regularisation of inverse problems. The main idea is to impose restrictions on the allowable solutions. A penalty term may be devised that favours smooth solutions, or perhaps solutions with a small norm. The optimal $\nu$ then minimises some combination of the least-squares misfit and the penalty term.

While such an optimisation-based formulation is intuitively pleasing, actually finding an optimal $\nu$ is by no means straightforward. There are many different optimisation algorithms available and all have their pros and cons. Vogel (2002) gives a concise overview of the computational tools that are available to solve such least-squares optimisation problems. Finally, it should be mentioned that other formulations of inverse problems exist that do not rely on optimisation of a norm. 
Such direct methods are usually applicable only to a specific class of problems and will not be discussed further.

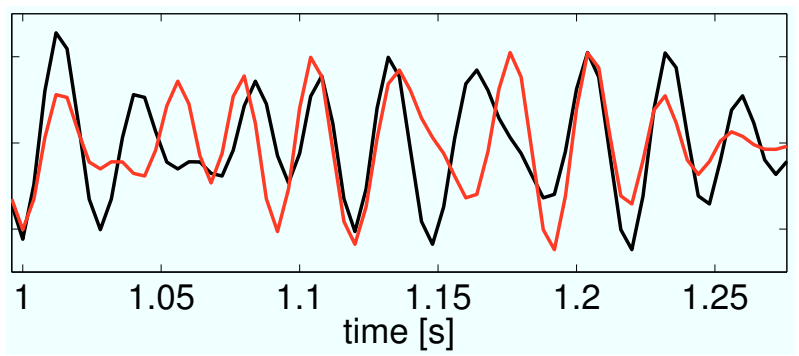

Figure 1.3. Illustration of the loop-skipping problem. A trace of the data from figure 1.2 is shown is black. The same trace, shifted by $0.024 \mathrm{~s}$ is shown in red. The partial fit of the trace with the shifted one causes a local minimum in the least-squares misfit.

\subsection{This thesis}

In this thesis we consider the inversion of seismic data in the constant density acoustic approximation. It turns out that the above mentioned least-squares formulation of the inverse problem is not optimal. A lot of prior information on the subsurface structure is needed to be able to get a reasonable result. In particular, such a-priori information takes the form of a slowly varying subsurface velocity. In other words, this formulation is suitable for migration and inversion, but not for velocity analysis. The basic rationale is this. The slowly varying velocity controls the traveltimes of the seismic waves. Since these waves are usually lacking low frequency content, a wrong traveltime may result in loop-skipping between the modelled and observed waveforms. This is illustrated in figure 1.3. The least-squares misfit exhibits local minima, making it less suitable for automatic optimisation. The subject of this thesis is the formulation of a data-misfit criterion that measures the kinematic difference between the wavefields and is amenable to automatic optimisation.

This thesis consists of an introductory chapter and several chapters written as selfcontained - sometimes published - papers, followed by discussion and recommendations for future research. The introductory section sketches the mathematical framework of the seismic inverse problem and briefly reviews several existing methods to infer a velocity model from the data. At the same time it serves as a guide through the rest of the thesis, explaining the connection between the chapters. 


\section{2 \\ Modelling and inversion of seismic data}

Abstract. In this chapter we briefly review the modelling and inversion of seismic data. It serves as a guide through the rest of the thesis, which consists of separately readable, sometimes published, chapters. 


\subsection{Modelling of seismic data in the acoustic approximation}

We assume that wave propagation in the earth is governed by the acoustic wave equation. In terms of the acoustic potential, $u$, as a function of time $t \in \mathbb{R}$ and position $\mathbf{x} \in \Omega \subseteq \mathbb{R}^{3}$ the acoustic wave equation reads (see Wapenaar \& Berkhout, 1989, for example):

$$
\underbrace{\left[\frac{1}{c(\mathbf{x})^{2}} \frac{\partial^{2}}{\partial t^{2}}-\rho(\mathbf{x}) \nabla \cdot\left(\frac{1}{\rho(\mathbf{x})} \nabla\right)\right]}_{\mathcal{L}[c]} u(t, \mathbf{x})=S(t, \mathbf{x}),
$$

where $c$ gives the soundspeed, $\rho$ the mass density, and $S$ represents a volume source. From the acoustic potential we may derive the pressure: $p=\frac{\partial u}{\partial t}$ and the particle velocity $\mathbf{v}=\rho^{-1} \nabla u$. Care must be taken to impose proper initial and boundary conditions. First of all we must ensure causality, that is, we want energy radiating out from the source from $t=0$ onwards. For example: $u(t<0, \mathbf{x})=0$. For marine acquisition, the ocean surface is usually modeled by imposing $u=0$ at the free surface. For the subsequent exposition we assume that such a boundary condition is not in effect. Moreover, we will use the acoustic potential rather than the physical quantities pressure or particle velocity to represent the seismic wavefield. Note however, that the pressure satisfies the same wave equation with $\frac{\partial S}{\partial t}$ as source.

\section{Rock properties}

Typically, velocities range from $1-6 \mathrm{~km} / \mathrm{s}$ and densities from $1-3 \mathrm{~kg} / \mathrm{m}^{3}$. Table 2.1 gives some specific values for various types of rock.

$\begin{array}{ccc} & \text { velocity }[\mathrm{km} / \mathrm{s}] & \text { density }\left[\mathrm{g} / \mathrm{cm}^{3}\right] \\ \text { Sandstone } & 5.5-9.0 & 2.0-2.6 \\ \text { Shale } & 1.8-4.9 & 2.0-2.7 \\ \text { Limestone } & 6.4-7.0 & 2.5-2.8 \\ \text { Salt } & 4.6 & 2.1-2.2 \\ \text { Basalt } & 4.5 & 2.7-3.1 \\ \text { Granite } & 6.1 & 2.75\end{array}$

Table 2.1. Some rock properties, from Sheriff \& Geldart (1982, table 5.2)

\section{Seismic sources}

A widely used representation of the source assumes an isotropic point radiator with time signature $f(t)$ :

$$
S(t, \mathbf{x})=f(t) \delta(\mathbf{x})
$$


The useable frequency-band of seismic data is typically $5-100 \mathrm{~Hz}$, depending on the source used. Very low frequencies are absent because of the very high amount of energy needed to generate them, although recent developments in acquisition technology are changing this. High frequencies are absent because of attenuation. An often-used source signature in synthetic studies is the Ricker wavelet (Kanasewich, 1985):

$$
f(t) \propto\left(1-2 t^{2}\right) \exp \left[-t^{2}\right]
$$

which can be dilated to have a desired peak frequency. Figure 2.1 depicts the Ricker wavelet and its spectrum.

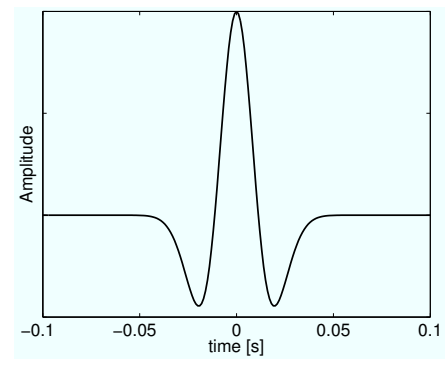

(a)

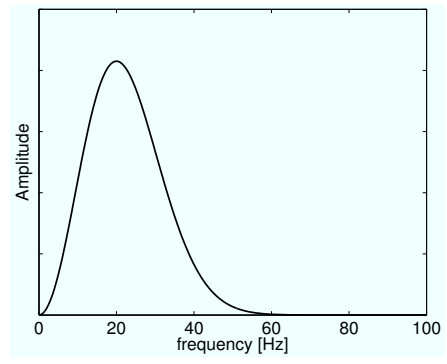

(b)

Figure 2.1. (a) Ricker wavelet with a peak frequency of $20 \mathrm{~Hz}$ and its spectrum (b).

\section{The seismic experiment}

Data are recorded by geophones, which are positioned at or just below the surface. Their position may be fixed or relative to the source position, as is the case in a marine survey. The experiment is repeated for various source positions. To indicate the dependency of the data on the source position explicitly we denote the wavefield as $u\left(t, \mathbf{x}, \mathbf{x}^{\prime}\right)$, which in case of a point source, obeys the following wave equation

$$
\mathcal{L}[c] u\left(t, \mathbf{x}, \mathbf{x}^{\prime}\right)=f(t) \delta\left(\mathbf{x}-\mathbf{x}^{\prime}\right) .
$$

The seismic data, $d$, are a restriction of $u$ to $\left\{\left(t, \mathbf{x}, \mathbf{x}^{\prime}\right) \mid t \in(0, T), \mathbf{x} \in \Omega_{R}, \mathbf{x}^{\prime} \in \Omega_{S}\right\}$, where $T$ denotes the recording time and $\Omega_{R}, \Omega_{S}$ are typically two-dimensional subspaces of $\Omega$ (the surface). We introduce the restriction operator $\mathcal{S}$ such that $d=\mathcal{S} u$. Typically, data are recorded along lines of a few kilometres long for 1000's of different source positions. Recently, 'wide-azimuth' acquisition setups are being used, which aim at recording larger offsets and getting better azimuthal coverage.

In the following, we assume unit density and an isotropic point source with known source signature and summarize solving the wave equation and subsequent restriction for a given velocity $c$ as

$$
d\left(t, \mathbf{x}_{s}, \mathbf{x}_{r}\right)=\mathcal{F}[c] .
$$


We will refer to $\mathcal{F}$ as the modelling operator.

\subsubsection{Linearization}

We introduce $c=c_{0}+\delta c$ and $u=u_{0}+\delta u$. With this we can write equation (2.4) as a system of 2 coupled PDE's:

$$
\begin{aligned}
\mathcal{L}\left[c_{0}\right] u_{0}\left(t, \mathbf{x}, \mathbf{x}^{\prime}\right) & =f(t) \delta\left(\mathbf{x}-\mathbf{x}^{\prime}\right) \\
\mathcal{L}\left[c_{0}\right] \delta u\left(t, \mathbf{x}, \mathbf{x}^{\prime}\right) & =\frac{2 \delta c(\mathbf{x})}{c_{0}(\mathbf{x})^{3}} \frac{\partial^{2}}{\partial t^{2}}\left(u_{0}+\delta u\right) .
\end{aligned}
$$

We apply the Born approximation, which amounts to assuming that the perturbations are small (i.e., neglecting $\delta c \times \delta u$ terms). With this we get a system in which the perturbation $\delta c$ appears linearly:

$$
\begin{aligned}
\mathcal{L}\left[c_{0}\right] u_{0}\left(t, \mathbf{x}, \mathbf{x}^{\prime}\right) & =f(t) \delta\left(\mathbf{x}-\mathbf{x}^{\prime}\right) \\
\mathcal{L}\left[c_{0}\right] \delta u\left(t, \mathbf{x}, \mathbf{x}^{\prime}\right) & =\frac{2 \delta c(\mathbf{x})}{c_{0}(\mathbf{x})^{3}} \frac{\partial^{2} u_{0}}{\partial t^{2}}\left(t, \mathbf{x}, \mathbf{x}^{\prime}\right) .
\end{aligned}
$$

Given Green's function, $G_{0}$, satisfying:

$$
\mathcal{L}\left[c_{0}\right] G_{0}=\delta\left(\mathbf{x}-\mathbf{x}^{\prime}\right) \delta(t),
$$

the incident wavefield, $u_{0}$, is given by

$$
u_{0}\left(t, \mathbf{x}, \mathbf{x}^{\prime}\right)=\int \mathrm{d} t^{\prime} f\left(t^{\prime}\right) G_{0}\left(t-t^{\prime}, \mathbf{x}, \mathbf{x}^{\prime}\right),
$$

and the scattered wavefield is given by:

$$
\begin{aligned}
\delta u\left(t, \mathbf{x}, \mathbf{x}^{\prime}\right) & =\int \mathrm{d} t^{\prime} \int \mathrm{d} \mathbf{x}^{\prime \prime}\left(\frac{\partial^{2} u_{0}}{\partial t^{\prime 2}}\left(t^{\prime}, \mathbf{x}^{\prime}, \mathbf{x}^{\prime \prime}\right) \frac{2 \delta c\left(\mathbf{x}^{\prime}\right)}{c_{0}\left(\mathbf{x}^{\prime}\right)^{3}}\right) G_{0}\left(t-t^{\prime}, \mathbf{x}^{\prime \prime}, \mathbf{x}\right) \\
& =\int \mathrm{d} t^{\prime} \int \mathrm{d} t^{\prime \prime} \int \mathrm{d} \mathbf{x}^{\prime \prime} \frac{2 \delta c\left(\mathbf{x}^{\prime}\right)}{c_{0}\left(\mathbf{x}^{\prime}\right)^{3}} G_{0}^{\prime \prime}\left(t^{\prime}-t^{\prime \prime}, \mathbf{x}^{\prime}, \mathbf{x}^{\prime \prime}\right) f\left(t^{\prime \prime}\right) G_{0}\left(t-t^{\prime}, \mathbf{x}^{\prime \prime}, \mathbf{x}\right)
\end{aligned}
$$

We introduce the linearized modelling operator $\delta \mathcal{F}\left[c_{0}\right]$ and corresponding linearized data $\delta d=\delta \mathcal{F}\left[c_{0}\right] \delta c$, so that $d=\mathcal{F}\left[c_{0}+\delta c\right] \approx \mathcal{F}\left[c_{0}\right]+\delta \mathcal{F}\left[c_{0}\right] \delta c$.

For $\delta d$ to represent actual seismic data with any kind of accuracy, we need the scales to separate. That is, the background velocity must be scatter-free in the seismic frequency band. Also, multiple scattering, diving waves and surface waves are ignored in this approach. Basically, we need $\mathcal{F}\left[c_{0}\right]=0$. Despite its apparent shortcomings, the Born approximation is the basis for most of the processing and interpretation tools that are being used with great success in exploration geophysics. 


\subsubsection{Asymptotic solution of the wave equation}

An asymptotic solution to the wave equation is widely-used for analysis, interpretation and processing of seismic data. While we focus on wave-equation modeling of the data in this thesis, we use the asymptotic expressions presented below for analysis. A derivation and more elaborate discussion of the following can be found in many excellent text books such as Bleistein (1984); Červený (2001); Bleistein et al. (2001).

We seek a solution of equation (2.10) of the form

$$
v\left(t, \mathbf{x}, \mathbf{x}^{\prime}\right)=\sum_{k \geq 0} \int \mathrm{d} \omega \omega^{m-k} A_{k}\left(\mathbf{x}, \mathbf{x}^{\prime}\right) \exp \left[\imath \omega\left(t-T\left(\mathbf{x}, \mathbf{x}^{\prime}\right)\right)\right]
$$

where $\omega$ denotes frequency and $T$ and $A_{k}$ represent the traveltime and amplitude of the waves. The parameter $m$ depends on the dimension $n: m=(n-3) / 2$. This is sometimes referred to as the WKBJ (Wentzel-Kramers-Brillouin-Jeffreys) trial solution or Debye series. For $T$ and the leading-order amplitude term $A_{0}$ we find

$$
\begin{aligned}
0 & =-\omega^{m+2}\left(\frac{1}{c_{0}^{2}}-\nabla T \cdot \nabla T\right) A_{0} \exp [\imath \omega(t-T)] \\
& +\imath \omega^{m+1}\left(2 \nabla A_{0} \cdot \nabla T+A_{0} \nabla^{2} T\right) \exp [\imath \omega(t-T)] \\
& -\omega^{m}\left(\nabla^{2} A_{0}\right) \exp [\imath \omega(t-T)]
\end{aligned}
$$

This yields the well-known eikonal and transport equations:

$$
\begin{aligned}
\nabla T \cdot \nabla T & =\frac{1}{c_{0}^{2}}, \\
\nabla \cdot\left(A_{0}^{2} \nabla T\right) & =0 .
\end{aligned}
$$

The corresponding asymptotic Green function is then given by

$$
G_{0}\left(t, \mathbf{x}, \mathbf{x}^{\prime}\right)=\int \mathrm{d} \omega \omega^{m} A_{0}\left(\mathbf{x}, \mathbf{x}^{\prime}\right) \exp \left[\imath \omega\left(t-T\left(\mathbf{x}, \mathbf{x}^{\prime}\right)\right)\right]+\mathcal{O}\left(\omega^{m-1}\right) .
$$

Such a representation is only possible in the absence of caustics, however. In the presence of caustics, the amplitude term becomes singular in this representation and one should move to a higher-dimensional representation.

In case of constant velocity we find

$$
\begin{aligned}
T\left(\mathbf{x}, \mathbf{x}^{\prime}\right) & =\left\|\mathbf{x}-\mathbf{x}^{\prime}\right\|_{2} / c_{0}, \\
A_{0}\left(\mathbf{x}, \mathbf{x}^{\prime}\right) & =1 /\left\|\mathbf{x}-\mathbf{x}^{\prime}\right\|_{2}
\end{aligned}
$$

To solve the eikonal and transport equations for variably velocity, it is common practice to employ the method of characteristics. Upon introducing the slowness 
$\mathbf{p}=\nabla T$, the eikonal equation can be written as a system of ODEs

$$
\begin{aligned}
& \frac{\mathrm{d} \mathbf{p}}{\mathrm{d} s}=\nabla\left(c_{0}^{-2}\right), \\
& \frac{\mathrm{d} \mathbf{x}}{\mathrm{d} s}=\mathbf{p}, \\
& \frac{\mathrm{d} T}{\mathrm{~d} s}=c_{0}^{-2} .
\end{aligned}
$$

Note that this representation is not unique and different parameterizations are often used for specific goals. Optimal parameterization of the ODEs, solution strategies and analysis are the subject of ray-theory.

\section{Layered media}

For a laterally invariant velocity, $c_{0} \equiv c_{0}(z)$, we have a constant horizontal slowness $p_{x}$. We consider rays propagating in the $x-z$ plane only, hence $p_{y}=0$. In terms of horizontal slowness $p_{x}$ and depth $z$ we then have a vertical slowness

$$
p_{z}\left(z, p_{x}\right)= \pm \sqrt{c(z)^{-2}-p_{x}^{2}}
$$

and

$$
\begin{aligned}
T\left(z, p_{x}\right) & =\int_{0}^{z} \mathrm{~d} z^{\prime} \frac{1}{c\left(z^{\prime}\right) \sqrt{1-p_{x}^{2} c\left(z^{\prime}\right)^{2}}}, \\
x\left(z, p_{x}\right) & =\int_{0}^{z} \mathrm{~d} z^{\prime} \frac{p_{x} c\left(z^{\prime}\right)}{\sqrt{1-p_{x}^{2} c\left(z^{\prime}\right)^{2}}} .
\end{aligned}
$$

For the amplitude term, which accounts for geometrical spreading, we find (see Črvený, 2001)

$$
\begin{aligned}
A\left(z, p_{x}\right) & =\sqrt{\frac{c(0) p_{x}}{p_{z}\left(0, p_{x}\right)}}\left(c(z) p_{z}\left(z, p_{x}\right) x\left(z, p_{x}\right) \frac{\partial x}{\partial p_{x}}\left(z, p_{x}\right)\right)^{-\frac{1}{2}} \\
& =\sqrt{p_{x}}\left(\sqrt{1-p_{x}^{2} c(0)^{2}} \sqrt{1-p_{x}^{2} c(z)^{2}} x\left(z, p_{x}\right) \frac{\partial x}{\partial p_{x}}\left(z, p_{x}\right)\right)^{-\frac{1}{2}}
\end{aligned}
$$

where

$$
\frac{\partial x}{\partial p_{x}}\left(z, p_{x}\right)=\int_{0}^{z} \mathrm{~d} z^{\prime} \frac{c\left(z^{\prime}\right)}{\left(1-p_{x}^{2} c\left(z^{\prime}\right)^{2}\right)^{3 / 2}} .
$$

For small $p_{x}$ we find

$$
\begin{aligned}
& T\left(z, p_{x}\right)=\int_{0}^{z} \mathrm{~d} z^{\prime} \frac{1}{c\left(z^{\prime}\right)}+\frac{p_{x}^{2}}{2} \int_{0}^{z} \mathrm{~d} z^{\prime} c\left(z^{\prime}\right)+\mathcal{O}\left(p_{x}^{4}\right) \\
& x\left(z, p_{x}\right)=p_{x} \int_{0}^{z} \mathrm{~d} z^{\prime} c\left(z^{\prime}\right)+\mathcal{O}\left(p_{x}^{2}\right) \\
& A\left(z, p_{x}\right)=\frac{1}{\int_{0}^{z} \mathrm{~d} z^{\prime} c\left(z^{\prime}\right)}+p_{x}^{2}\left(\frac{c(0)^{2}+c(z)^{2}}{4 \int_{0}^{z} \mathrm{~d} z^{\prime} c\left(z^{\prime}\right)}+\frac{\int_{0}^{z} \mathrm{~d} z^{\prime} c\left(z^{\prime}\right)^{3}}{\left(\int_{0}^{z} \mathrm{~d} z^{\prime} c\left(z^{\prime}\right)\right)^{2}}\right)+\mathcal{O}\left(p_{x}^{4}\right)
\end{aligned}
$$


Introducing the zero-offset traveltime, $\tau_{0}$, and $\gamma_{0}$, which are given by

$$
\begin{aligned}
& \tau_{0}(z)=\int_{0}^{z} \mathrm{~d} z^{\prime} \frac{1}{c\left(z^{\prime}\right)}, \\
& \gamma_{0}(z)=\left(\int_{0}^{z} \mathrm{~d} z^{\prime} c\left(z^{\prime}\right)\right)^{-1},
\end{aligned}
$$

we obtain

$$
\begin{aligned}
& T(z, x) \approx \tau_{0}(z)+\frac{x^{2}}{2} \gamma_{0}(z), \\
& A(z, x) \approx \gamma_{0}(z)\left(1+\left(\gamma_{0}(z) \frac{x}{2}\right)^{2}\left(c(0)^{2}+c(z)^{2}+4 \gamma_{0}(z) \int_{0}^{z} \mathrm{~d} z^{\prime} c\left(z^{\prime}\right)^{3}\right)\right)
\end{aligned}
$$

This approximation of the traveltime called the NMO traveltime and is often used in the following form (Dix, 1955)

$$
T_{\mathrm{NMO}}\left(t_{0}, x\right)=\sqrt{t_{0}^{2}+x^{2} / c_{\mathrm{RMS}}\left(t_{0}\right)^{2}},
$$

which can be derived from equation (2.33) by parameterizing depth with zero-offset traveltime. The RMS velocity is then related to the physical velocity by

$$
c_{\mathrm{RMS}}\left(t_{0}\right)=\sqrt{\frac{1}{t_{0}} \int_{0}^{t_{0}} \mathrm{~d} t^{\prime} c\left(z\left(t^{\prime}\right)\right)^{2}},
$$

where $z\left(t_{0}(z)\right)=z$.

\subsection{Sensitivity analysis}

Which parts of the data are sensitive to which components of the velocity? Physical intuition suggests that the traveltimes of the data are mostly sensitive to variations in the background velocity while the amplitudes of the data are mostly sensitive to variations in the reflectivity. Wang \& Pratt (1997) confirm this by a numerical study. In this section we present an analysis based on the Born and Rytov approximations that justifies these statements and explores the trade-off between frequency content and aperture. We will see that traveltime and amplitude are nicely complementary and may - in special cases - provide a complete spectral coverage of the velocity perturbation. When a serious lack of low frequencies and large scattering angles occurs, however, the intermediate wavenumbers of the velocity may be undetermined. This is a well-known problem in seismic inversion (Claerbout, 1984; Jannane et al., 1989).

\subsubsection{Amplitude sensitivity via the Born approximation}

In the Born approximation, we linearize the amplitude of the Green function w.r.t. a velocity perturbation: $r=2 c_{0}^{-3} \delta c$. We derived earlier that (cf. equation (2.12))

$$
\widehat{\delta G}\left(\omega, \mathbf{x}_{s}, \mathbf{x}_{r}\right)=\omega^{2} \int \mathrm{d} \mathbf{x} r(\mathbf{x}) \hat{G}\left(\omega, \mathbf{x}_{s}, \mathbf{x}\right) \hat{G}\left(\omega, \mathbf{x}, \mathbf{x}_{r}\right),
$$


where $\hat{\imath}$ denotes the Fourier transform. Upon substituting the asymptotic Green function, we find that

$$
\widehat{\delta G}\left(\omega, \mathbf{x}_{s}, \mathbf{x}_{r}\right)=\omega^{2} \int \mathrm{d} \mathbf{x} r(\mathbf{x}) A\left(\mathbf{x}_{s}, \mathbf{x}\right) A\left(\mathbf{x}, \mathbf{x}_{r}\right) \exp \left[\imath \omega\left(T\left(\mathbf{x}, \mathbf{x}_{s}\right)+T\left(\mathbf{x}, \mathbf{x}_{r}\right)\right)\right](
$$

Next, we linearize the traveltime locally around a scattering point $\mathbf{x}_{0}$ :

$$
T\left(\mathbf{x}, \mathbf{x}^{\prime}\right) \approx T\left(\mathbf{x}_{0}, \mathbf{x}^{\prime}\right)+\left(\mathbf{x}-\mathbf{x}_{0}\right) \cdot \mathbf{p}\left(\mathbf{x}_{0}, \mathbf{x}^{\prime}\right) .
$$

We then identify equation (2.38) as a Fourier transform of $r$ with wavenumber:

$$
\mathbf{k}=\omega\left(\mathbf{p}\left(\mathbf{x}_{0}, \mathbf{x}_{s}\right)+\mathbf{p}\left(\mathbf{x}_{0}, \mathbf{x}_{r}\right)\right)
$$

We write the slowness as $\mathbf{p}=\cos \theta \mathbf{n} / c\left(\mathbf{x}_{0}\right)$, where $\mathbf{n}$ is the unit vector normal to the reflector and $\theta$ is the angle between the ray and the normal. Using Snell's law we find

$$
\mathbf{k}=\frac{2 \omega}{c\left(\mathbf{x}_{0}\right)} \cos \theta \mathbf{n}
$$

where $\theta$ is the scattering angle and $\mathbf{n}$ the unit vector normal to the reflector. Hence, we need large scattering angles (large aperture) to sample the low wavenumbers via high-frequency data.

\subsubsection{Phase sensitivity via the Rytov approximation}

In the Rytov approximation, we linearize the phase of the Green function:

$$
T[c+\delta c] \approx T[c]+\delta T[c]
$$

where $\delta T$ satisfies a linearized Eikonal equation:

$$
\nabla \delta T \cdot \nabla T=-\delta c\left(\mathbf{x}-\mathbf{x}^{\prime}\right) / c_{0}^{3}
$$

The linearized Green function is then given by

$$
\widehat{\delta G}[c] \approx \frac{\widehat{G}[c+\delta c]}{\widehat{G}[c]}=A[c] \exp [\imath \omega \delta T[c]]
$$

When we use a constant background velocity, the rays are straight lines and the traveltime perturbations are given by a Radon transform of the velocity perturbation. For simplicity, we consider rays in the $\left(x_{1}, x_{3}\right)$ plane and a velocity that depends on $x_{1}, x_{3}$ only. Introducing polar coordinates $\mathbf{x}=\left(\rho \cos \theta, x_{2}, \rho \sin \theta\right), \mathbf{x}^{\prime}=$ $\left(\rho^{\prime} \sin \theta, x_{2},-\rho^{\prime} \cos \theta\right)$ yields:

$$
\delta T\left(\rho^{\prime}, \theta\right)=-c_{0}^{-2} \int_{0}^{R_{\max }} \mathrm{d} \rho \delta c\left(\rho \sin \theta+\rho^{\prime} \cos \theta, \rho \cos \theta-\rho^{\prime} \sin \theta\right) .
$$


Figure 2.2 illustrates the geometry. We can now relate the Fourier transform of $\delta T$ w.r.t. $\rho$ to the 2D Fourier transform of $\delta c$ via the Fourier slice theorem (cf Gaskill, 1978):

$$
\widehat{\delta T}(2 k, \theta)=\widehat{\delta c}(k \cos \theta,-k \sin \theta) .
$$

Because of the limitation of having sources and receivers at the surface we have $\theta \in(-\pi / 2, \pi / 2)$.

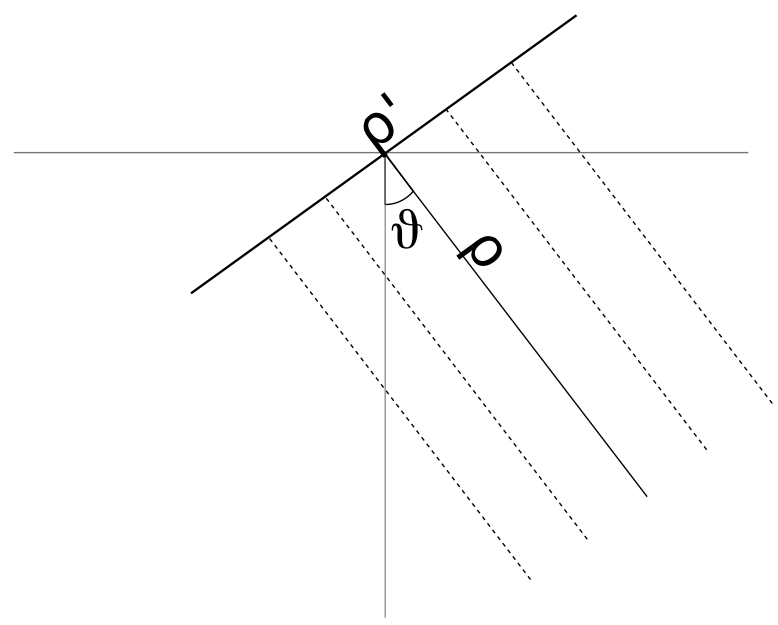

Figure 2.2. Geometry of traveltime tomography. In a constant velocity medium the rays are straight lines at an angle $\theta$. The traveltime perturbations as a function of $\theta$ and $\rho^{\prime}$ are given by integration of the slowness perturbation along $\rho$.

\section{Layered media}

To get some feeling for what the above analysis means in practice, we study the simplest case: horizontally layered media. We consider retrieving a horizontally layered velocity perturbation, $\delta c\left(x_{3}\right)$, given a constant background velocity $c_{0}$. The amplitudes are sensitive to the wavenumbers:

$$
k_{3}=\frac{2 \omega}{c_{0}} \cos \theta
$$

For the traveltime data to be available, we need reflections. Let's denote the traveltime information generated by a single reflector at (unknown!) depth $z$ with

$$
T_{z}(\rho, \theta)=\delta T(\rho, \theta)+\delta T(\rho,-\theta) .
$$

The depth of the reflector for a given velocity can be determined from $T_{z}(t, 0)$. The wavelength that can be retrieved is then given by:

$$
k_{3}=k \sin \theta / 2 \text {. }
$$


The maximal $k$ that is available depends on the sampling of $\rho$, which in turn depends on the sampling of the reflectors. Assuming a uniform coverage of horizontal reflectors with distance $\Delta z$, we have $\Delta \rho=\Delta z \cos \theta$, leading to $k_{\max }=\pi /(\Delta z \cos \theta)$. The sampling of the reflectors, on the other hand, depends on the frequency content of the data. Assuming that we cannot distinguish two reflectors that are less than a wavelength apart, leads us to $\Delta z_{\min }=\pi c_{0} /\left(\omega_{\max } \cos \theta\right)$. Hence, $k_{\max }=\omega_{\max } /\left(c_{0}\right)$. We find that the wavenumbers that can be retrieved from the amplitudes are given by

$$
\frac{2 \omega_{\min }}{c_{0}} \cos \theta \leq k_{3} \leq \frac{2 \omega_{\max }}{c_{0}} \cos \theta,
$$

while the wavenumbers that can be recovered from the traveltimes are given by

$$
0 \leq k_{3} \leq \frac{\omega_{\max }}{2 c_{0}} \sin \theta
$$

The wavenumber coverage from amplitude and traveltime are complementary for small angles. This explains the low sensitivity to the intermediate features, mentioned before. Figure 2.3 shows the different curves in the $\left(k_{3}, \theta\right)$-plane for typical seismic acquisition parameters.

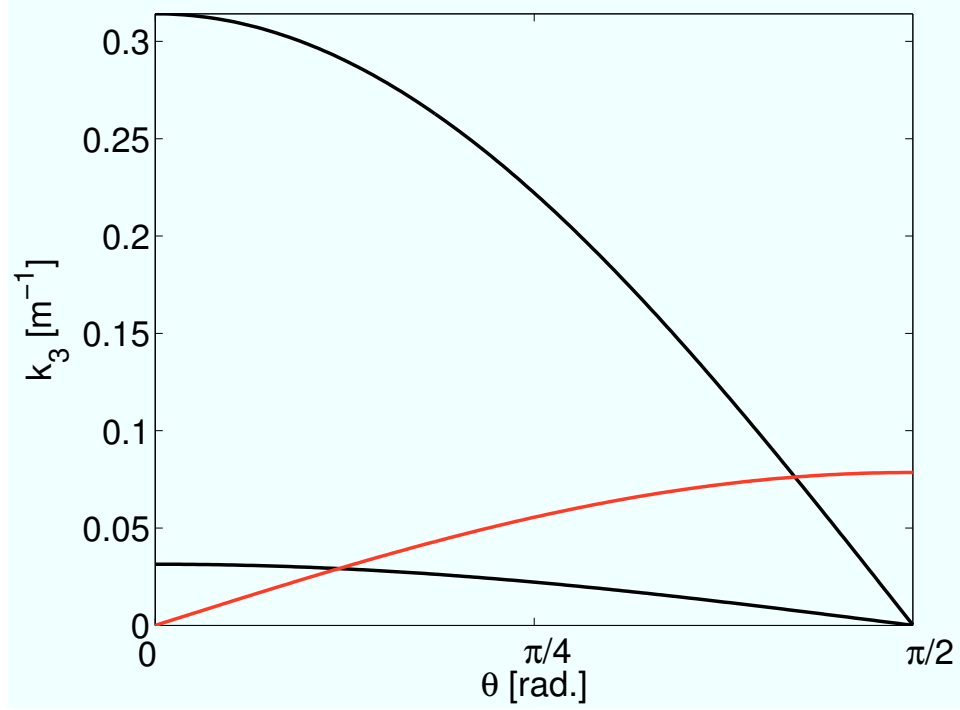

Figure 2.3. Wavenumber coverage of traveltime (red) and amplitude (black) information assuming a horizontally layered velocity perturbation and a constant background velocity of $2 \mathrm{~km} / \mathrm{s}$ and frequencies between $5-50 \mathrm{~Hz}$. See also equation (2.51) and equation (2.52). 


\subsubsection{Uniqueness}

The above analysis for layered media suggests that we need an infinite amount of reflectors, infinitely high frequency and at least two angles to retrieve a spectrally complete velocity. This has actually been proven rigorously by Symes (1991). To the author's best knowledge no such results exist for non-layered velocity models. For the transmission case, the Fourier slice theorem suggests that we need measurements for all angles to uniquely determined the velocity. However, this analysis is based on a linearization around a constant background. In general, the rays are not straight lines at all and depend on the velocity perturbation. Again, to the author's best knowledge, no results exist for the kind of media that one encounters in seismic applications.

\subsection{Inversion of seismic data}

Having discussed the modelling of seismic data and the sensitivity of the data to velocity perturbations, we come to the inversion of seismic data:

\section{Given observed data, $\bar{d}$, find a subsurface model for which} the modelled data, $d$, minimises the misfit functional $\phi[\bar{d}, d]$

This may be interpreted in several ways, depending on the definition of 'matching the data' and 'modelled data'.

When the modelled data is a (numerical) solution of the wave equation and with matching we mean minimizing the difference in some norm, we arrive at non-linear data-fitting procedure called Full Waveform Inversion (FWI) which is discussed in section 2.3.1. Alternatively, the data may be modelled with the Born approximation. The unknown quantity is then taken to be the velocity perturbation w.r.t. a given background velocity. This is called migration and can be solved using either wave-equation or ray-based methods. As opposed to FWI, this leads to a linear data-fitting procedure, which is discussed in section 2.3.2. Finally, we may want to match the traveltimes. To this end several tomographic procedures, labelled velocity analysis or (reflection) tomography, have been developed. These will be briefly discussed in sections 2.3.3, 2.3.4, and 2.3.5.

\subsubsection{Full waveform inversion}

Classical FWI aims at finding the velocity model that minimises the difference between the modelled and observed data in a least-squares sense:

$$
c^{*}=\underset{c}{\operatorname{argmin}}\|\mathcal{F}[c]-d\|_{2}^{2} .
$$

This approach was introduced in the geophysical community by Tarantola \& Valette (1982). A gradient-based optimisation algorithm can be employed to find the minimizer. The gradient of the objective functional can be efficiently calculated via the adjoint-state technique (Tarantola \& Valette, 1982; Lailly, 1983; Plessix, 2006) and 
is given by

$$
g(\mathbf{x}) \propto \int \mathrm{d} t \int \mathrm{d} \mathbf{x}_{s} u\left(t, \mathbf{x}_{s}, \mathbf{x}\right) \frac{\partial^{2} v}{\partial t^{2}}\left(t, \mathbf{x}_{s}, \mathbf{x}\right),
$$

where

$$
\begin{aligned}
\mathcal{L}[c] u & =f \delta\left(\mathbf{x}-\mathbf{x}_{s}\right), \\
\mathcal{L}^{*}[c] v & =\mathcal{S}^{*}(\mathcal{F}[c]-d) .
\end{aligned}
$$

However, as the sensitivity analysis suggests, this approach can be problematic when the initial model is too far from the true model. The consensus is that the initial model has to explain the data to within half a wavelength for the lowest frequency. Otherwise, the gradient-based optimisation method may get stuck in a local minimum. While this problem has been observed in practice, little theory has been developed to support this.

Any relevant physics can in principle be incorporated in this approach and, when given a good initial model, may yield quantitative results with an unprecedented level of detail and fidelity.

Recent developments in acquisition have made it possible to record lower frequencies and longer offsets. Both may provide information on the slowly varying component of the velocity directly and different approaches have been developed to focus on the low frequencies (Bunks et al., 1995) or diving waves (Pratt \& Hicks, 1998) in the data. With these techniques spectacular results have been obtained in the last few years.

Other techniques to mitigate the local minima of the least-squares misfit functional have also been proposed. These involve using different norms, such as $L_{1}$ or hybrid norms such as the Huber norm, which reduces to the $L_{1}$ norm for large residuals and to the $L_{2}$ norm for small residuals (Crase et al., 1990; Guitton \& Symes, 2003). Another approach relies on extracting the phase difference from the data and matching the unwrapped phases in a least-squares sense (Shin \& Min, 2006). A recent development is the inversion in the Laplace (Shin \& Cha, 2008) and LaplaceFourier domain (Shin \& Cha, 2009).

For a more elaborate overview of FWI we refer to Virieux \& Operto (2009).

\subsubsection{Linearized inversion}

The goal of linearized inversion, or migration, is to obtain an estimate of the reflectivity $\delta c$, given the measured data $d$ and a velocity model $c_{0}$, such that $\delta F\left[c_{0}\right] \delta c \simeq d$. Since the data are 5 -dimensional for a full acquisition while the reflectivity is only 3 -dimensional, this problem is - usually- overdetermined. Furthermore, this formulation assumes that the data are consistent with the linearized modelling operator. This means that the surface wave, diving waves/refractions and multiple reflections have to be absent. Basic techniques to remove these unwanted events from the data can be found in Yilmaz \& Chambers (1984). Assuming for the moment that there 
is indeed a $\delta c$ such that $\delta \mathcal{F}\left[c_{0}\right] \delta c=d$, we may naively write:

$$
\delta c=(\underbrace{\delta \mathcal{F}^{*}\left[c_{0}\right] \delta \mathcal{F}\left[c_{0}\right]}_{\mathcal{N}\left[c_{0}\right]})^{-1} \delta \mathcal{F}^{*}\left[c_{0}\right] d,
$$

where $\delta \mathcal{F}^{*}$ is the adjoint of the linearized modelling operator. The operator $\mathcal{N}$ is often referred to as the normal operator or Hessian. Asymptotically, the normal operator turns out to be a pseudo-differential operator of order two (cf Beylkin, 1984). Roughly, this means that

$$
\mathcal{N} \delta c(\mathbf{x}) \simeq \alpha(\mathbf{x}) \Delta \delta c,
$$

where $\alpha$ is a smooth positive function. This implies that $\delta \mathcal{F}^{*}\left[c_{0}\right] d$ is already a usable approximation of $\delta c$, since the normal operator acts only as a filter and scaling factor. The exact conditions under which the normal operator is invertible have been extensively studied (Beylkin, 1984, 1985; ten Kroode et al., 1998; Stolk \& de Hoop, 2006; de Hoop et al., 2009). Next, we will briefly review several methods that can be used to obtain an approximate solution of the linearized inverse problem, while we refer to Etgen et al. (2009) for an extensive, up-to-date overview of migration methods.

\section{Ray-based methods}

Upon substituting the asymptotic Green function the linearized modelling operator is a Generalized Radon Transform (GRT) of the reflectivity (omitting some amplitude factors):

$$
\delta d\left(t, \mathbf{x}_{s}, \mathbf{x}_{r}\right)=\int \mathrm{d} \omega \int \mathrm{d} \mathbf{x} \delta c(\mathbf{x}) \exp \left[\imath \omega\left(t-T\left(\mathbf{x}_{s}, \mathbf{x}\right)-T\left(\mathbf{x}, \mathbf{x}_{r}\right)\right)\right] .
$$

The GRT may be approximately inverted by applying the adjoint, as argued above. The traveltimes may be calculated via ray tracing. This leads to 'Kirchoff migration' (see Bleistein et al., 2001, for example). The approximate inverse of the Hessian may be obtained via calculation of the so-called Beylkin determinant, which arises when explicitly working out $\mathcal{N}$ and introducing appropriate coordinate transforms (Beylkin, 1984).

\section{Wave equation-based methods}

The linearized inverse problem may also be posed as a least-squares optimisation problem:

$$
\{\delta c\}=\underset{\delta c}{\operatorname{argmin}}\left\|\delta \mathcal{F}\left[c_{0}\right] \delta c-d\right\|_{2}^{2} .
$$

The formal gradient of the least-squares misfit functional w.r.t. $\delta c$ can again be calculated via the adjoint-state technique, as presented in equation (2.54). This leads to reverse-time migration and iterative migration (Mulder \& Plessix, 2004; Plessix \& Mulder, 2004). Such techniques are quickly becoming the industry standard as they better deal with lateral heterogeneity. They are also more sensitive to errors in the background velocity and wrong processing of the data, however. 


\subsubsection{Migration Velocity Analysis}

One way to obtain a suitable starting model for migration, linearized inversion or waveform inversion is migration velocity analysis. There are a number of ways to introduce the notion of migration velocity analysis (MVA) and an excellent review is given by Symes (2008b). Chapter 4 also gives an overview and compares different methods in the context of layered media.

The basic principle that underlies migration velocity analysis is that the redundancy of the data may be used to create several independent estimates of the reflectivity - recall that full-acquisition data are 5-D, while the reflectivity is 3-D. Assuming that the reflectivity does not change during the experiment, these images should more or less agree. Subsequently, different criteria may be derived to judge whether the images agree. The redundancy in the reflectivity estimate can be introduced in several ways. To most straightforward way is based on data-binning; several subsets of the data are used separately to form an image. A common way of binning is based on the midpoint-offset coordinates $\mathbf{x}_{m}=\frac{1}{2}\left(\mathbf{x}_{s}+\mathbf{x}_{r}\right)$ and $\mathbf{h}=\frac{1}{2}\left(\mathbf{x}_{s}-\mathbf{x}_{r}\right)$. Each constant-offset bin, $d_{\mathbf{h}}\left(t, \mathbf{x}_{m}\right)=d\left(t, \mathbf{x}_{m}-\mathbf{h}, \mathbf{x}_{m}+\mathbf{h}\right)$, is then used separately to produce a common-offset image:

$$
I_{\mathrm{co}}(\mathbf{x}, \mathbf{h})=\delta \mathcal{F}^{*}\left[c_{0}\right] d_{\mathbf{h}} .
$$

Basically, any migration method can be used to produce these images. Most commonly, however, these images are produced by some form of Kirchoff migration or NMO correction. For the correct velocity such a common-offset image should independent of offset. This is the basis for many (if not all) of the currently-used techniques for migration velocity analysis. Depending on the precise way of binning the data and whether or not depth is parameterized as vertical traveltime, such reflectivity estimates are called Common-Something-Gathers (CSGs), where Something may be midpoint, depth-point, ... The methods differ in the way the velocity is parameterized, the way of picking relevant events, the way the wave propagation is modelled, and so on. The basic principle, however, remains: find a velocity that flattens the CSGs. A disadvantage of these approaches is that artefacts may occur in the CSGs due to the binning when the underlying velocity varies strongly in the lateral direction (Stolk \& Symes, 2004; Stolk et al., 2009). That is, the CSGs may not be flat for the correct velocity.

Another redundant coordinate that is gaining popularity is the subsurface offset. In terms of the source and receiver wavefields, introduced earlier equation (2.54), the shot-geophone image is then given by

$$
I_{\mathrm{sg}}(\mathbf{x}, \Delta \mathbf{x})=\int \mathrm{d} t \int \mathrm{d} \mathbf{x}_{s} u_{0}\left(t, \mathbf{x}-\Delta \mathbf{x}, \mathbf{x}_{s}\right) \frac{\partial^{2} v_{0}}{\partial t^{2}}\left(t, \mathbf{x}+\Delta \mathbf{x}, \mathbf{x}_{s}\right) .
$$

Such images are better suited for wave-equation migration methods as the source and receiver wavefields are readily available. For the correct velocity we have

$$
I_{\mathrm{sg}}(\mathbf{x}, \Delta \mathbf{x}) \simeq r(\mathbf{x}) \delta(\Delta \mathbf{x}) .
$$

This was first introduced by Doherty \& Claerbout (1974). Stolk \& de Hoop (2006) showed that such an image is essentially free from artefacts. This reflectivity estimate may also be turned into a CSG via a Radon transform. 
A less-widely used alternative is to introduce a temporal shift Rickett \& Sava (2002); Sava \& Fomel (2006):

$$
I_{\mathrm{ts}}(\mathbf{x}, \Delta t)=\int \mathrm{d} t \int \mathrm{d} \mathbf{x}_{s} u_{0}\left(t-\Delta t, \mathbf{x}, \mathbf{x}_{s}\right) \frac{\partial^{2} v_{0}}{\partial t^{2}}\left(t+\Delta t, \mathbf{x}, \mathbf{x}_{s}\right) .
$$

This is fundamentally different from the previous approaches. While the surface and subsurface offset reflectivities have the same dimensionality as the data, the time-shift reflectivity does not. This turns out to be of vital importance, as we will see later. Also, the time-shift image does not have such nice focussing properties (Sava \& Fomel, 2006). A more elaborate overview and analysis of these methods is given in chapter 4 .

Next we briefly discuss several ways of exploiting the properties outlined above.

\section{Stackpower}

One way of judging whether the offset reflectivity is independent of offset is by relying on constructive interference to select the velocity that maximizes the stackpower:

$$
S(\mathbf{x})=\int \mathrm{d} \mathbf{h} I_{\mathrm{co}}(\mathbf{x}, \mathbf{h})
$$

A common way to implement this is called semblance analysis. The offset-dependent image is produced by NMO correction. The semblance is then calculated for several constant NMO velocities. The resulting semblance panel is then a function of velocity and traveltime. The NMO velocity as a function of depth is then extracted by picking the maxima in the semblance panel.

Toldi (1989) proposed to cast this as an optimisation problem to find the velocity that maximizes $\|S\|_{2}^{2}$. This misfit functional may exhibit local maxima due to constructive interference of nearby events, however, making it less suitable for optimisation using a gradient-based optimisation method.

\section{DSO}

An elegant way of measuring the flatness of the CSGs is to simply take the derivative in the redundant coordinate and was first proposed by Symes \& Carazzone (1991) for the surface-offset case. The corresponding misfit function, fittingly labelled differential semblance is given by

$$
\left\|\partial_{h} I_{\mathrm{co}}\right\|_{2}^{2}
$$

Analysis for layered models suggests that this misfit functional has no local minima and is essentially quadratic in the velocity error (Symes, 1999). An abundance of numerical evidence exists that this holds for laterally varying models (Chauris \& Noble, 2001; Mulder \& ten Kroode, 2002; Li \& Symes, 2007).

An alternative to differentiating w.r.t. to the redundant coordinate can be cooked 
up by considering the subsurface-offset image (or the lateral Fourier transform of any of the CSGs, for that matter). Shen et al. (2003) proposes to optimize

$$
\left\|W \cdot I_{\mathrm{sg}}\right\|_{2}^{2}, \quad W(\Delta \mathbf{h})=\Delta \mathbf{h},
$$

see also de Hoop et al. (2006); Shen \& Symes (2008).

All these different variants of DSO are interlinked in a framework that is sometimes referred to as the annihilator-based approach to velocity analysis. Basically they can be written as

$$
\left\|\mathcal{A}\left[c_{0}\right] \bar{d}\right\|_{2}^{2}
$$

where $\mathcal{A}$ is the annihilator, i.e., for the correct velocity model $\mathcal{A}\left[\bar{c}_{0}\right] \bar{d} \simeq 0$. Stolk \& Symes (2003) showed that they all lead to a misfit functional that behaves smoothly as a function of the velocity error. A key ingredient for this to be true is the invertibility of $\mathcal{A}$. Therefore, it is important that the generalized image has the same dimensionality as the data.

Some interesting developments in this area are the velocity continuation of the extended images (Duchkov et al., 2008; Duchkov \& de Hoop, 2009) and generalization of the principle to the non-linear case (Symes, 2008a).

\subsubsection{Reflection tomography}

In reflection tomography, one relies on fitting the traveltimes of selected events in the data directly. To this end, a fixed number of reflectors and the velocity are taken as unknowns. The traveltimes are predicted via raytracing, taking reflections into account, and these are to be matched to the picked traveltimes in the data. Updating the velocity is done by projecting the traveltime-difference back along the rays in the current velocity. Subsequently, the reflector positions are be updated to optimally fit the data with the new velocity and the cycle is repeated Bishop et al. (1985) describes an all-in-one approach of this nature. An extension of this method, called stereo tomography, that does not only try to fit the traveltimes but also the local slopes (slownesses) was proposed by Billette \& Lambaré (1998). This helps to distinguish events that arrive at the same time with a different slowness. An alternative way to view the reflection tomography problem comes from the linearized LS problem. Instead of just optimizing for $\delta c$, we can simultaneously vary $c_{0}$ and minimise:

$$
\left\{c_{0}, \delta c\right\}=\operatorname{argmin}\left\|\delta \mathcal{F}\left[c_{0}\right] \delta c-\delta d\right\|_{2}^{2} .
$$

This is a so-called separable least-squares problem and special algorithms have been devised to deal with these (Golub \& Pereyra, 1973). The basic idea is quite simple and consists of eliminating the linear unknown, in this case $\delta c$ by a projection onto the range of $\delta \mathcal{F}$ :

$$
\delta c\left[c_{0}\right]=\pi\left[c_{0}\right] \delta d,
$$

where the projection operator is given by

$$
\pi\left[c_{0}\right]=\mathcal{N}^{-1} \delta \mathcal{F}^{*} .
$$


This projection operator is also studied in the context of data continuation by de Hoop \& Uhlmann (2005). We may also view $\pi-I$ - where $I$ is the identity operator - as an annihilator, and in that sense it fits the general framework mentioned earlier. This leads to a modified LS problem:

$$
c_{0}^{*}=\underset{c_{0}}{\operatorname{argmin}}\left\|\left(\pi\left[c_{0}\right]-I\right) \delta d\right\|_{2}^{2} .
$$

This is essentially the Migration Based Traveltime Tomography (MBTT) approach, proposed by Clément et al. (2001). It was shown by Stolk \& Symes (2003), however, this particular annihilator does not lead to a smooth objective functional.

It turns out that the wavefields $\delta d$ and $\pi \delta d$ differ significantly in moveout when the velocity is not correct. However, when using the least-squares misfit, we are not directly fitting traveltimes. In fact, we show in chapter 4 that this approach is equivalent to the stackpower approach, explained above. We can use the projection operator to formulate a reflection tomography problem by using a different misfit functional, based on the correlation of the data and their projection. Detailed analysis of this approach in the context of horizontally layered media is the subject of chapter 4. First experiments with this method in a simplified setting are described in chapter 3. Application of the method to laterally varying velocities is described in chapter 6 .

\subsubsection{Transmission tomography}

In transmission tomography, one tries to infer a velocity model from measurements of transmitted (body) waves. Some examples are cross-well tomography, divingwave tomography and earth-quake tomography. The basic principle is still same: find a model that produces data which optimally fits the measured data. Again, we would like to focus on fitting the traveltimes of the data and hence it is natural to employ the correlation-based misfit functionals. In fact, the use of a correlation to extract a traveltime misfit seems to be first used for transmission tomography. Cara \& Leveque (1987), for example, describe a procedure to extract the traveltime difference from a correlation of the modeled and observed waveforms. The rationale behind this method is that the traveltime perturbation depends more linearly on the velocity perturbation. Of course, when we revert to ray-theory, we can immediately see that the traveltime and velocity perturbation are related linearly. The classical way of performing transmission tomography is based on ray-theory and relies on fitting the computed traveltimes to picked traveltimes. However, with the advent of routine digital recording of broadband data and the need to explore ever more complex areas the use of the full waveform and modeling of wave propagation through the wave equation is becoming the standard. A challenge here is to measure the traveltime difference between the modeled and observed waveform and to come up with a relation between the misfit and the velocity update. Luo \& Schuster (1991) describe such a procedure which is based on picking the maximum of the correlation of the waveforms. This has been de basis of many subsequent developments (Dahlen et al., 2000; de Hoop \& van der Hilst, 2005).

In chapter 5 we briefly review these conventional techniques and describe how the 
correlation-based criterion discussed in chapters 3 and 4 can be used in this setting.

\subsection{Summary}

We have briefly reviewed the modeling of seismic data in the constant-density acoustic approximation. While we will focus on using the wave equation to model the data, we include a brief review of the asymptotic approximation that is widely used. We will use this approximation later on for analysis. Then, described the sensitivity of the amplitude and phase of seismic data to both smooth and oscillatory velocity perturbations. We use the analysis to explain the trade-off between frequency content and aperture. For typical seismic experiments, amplitude and traveltime of the data provide information on different components of the velocity. Finally, we summarized different methods that are used to invert seismic data.

The overview of the thesis is as follows. In chapter 3 we introduce the use of a correlation of modeled and observed data for velocity analysis. To model the data, we use NMO traveltimes. In chapter 4 we analyze and compare the data-correlation methods to the conventional migration velocity analysis methods using the asymptotic formulas described in section 2.1.2. In chapter 5 we discuss the application of the correlation-based misfit criteria to wave-equation transmission tomography, while in chapter 6 we elaborate on wave-equation reflection tomography. 


\title{
3 \\ Velocity analysis based on data correlation
}

T. van Leeuwen and W.A. Mulder. 2008. Geophysical Prospecting, 56(6):791-803

\begin{abstract}
Several methods exist to automatically obtain a velocity model from seismic data via optimization. Migration velocity analysis relies on an imaging condition and seeks the velocity model that optimally focuses the migrated image. This approach has been proven to be very successful. However, most migration methods use simplified physics to make them computationally feasible and herein lies the restriction of migration velocity analysis. Waveform inversion methods use the full wave equation to model the observed data and more complicated physics can be incorporated. Unfortunately, due to the band-limited nature of the data, the resulting inverse problem is highly non-linear. Simply fitting the data in a least-squares sense by using a gradient-based optimization method is sometimes problematic. In this paper, we propose a novel method that measures the amount of focusing in the data-domain rather than the image-domain. As a first test of the method, we include some examples for $1 D$ velocity models and the convolutional model.
\end{abstract}




\subsection{Introduction}

Most successful methods to obtain a velocity model from seismic data automatically try to focus the migrated image in some sense. This approach to velocity analysis is called migration velocity analysis (MVA) and has a long history. The underlying principle of MVA has not changed, but the precise way in which way the amount of focusing of the image is measured and the migration algorithms themselves have evolved. A few well-known methods found in this class are Differential Semblance Optimization (DSO), proposed by Symes \& Carazzone (1991), which uses flatness of image gathers as a measure, and methods based on a generalized imaging condition, proposed by Doherty \& Claerbout (1974) and elaborated on by many others (Yilmaz \& Chambers, 1984; Stork, 1992; MacKay \& Abma, 1992; Rickett \& Sava, 2002; Sava \& Biondi, 2004; Sava \& Fomel, 2006). These methods have been proven successful on data in which multiples are not too strong (Mulder \& ten Kroode, 2002; Shen et al., 2003; Dussaud \& Symes, 2005; Li \& Symes, 2007). A common factor in all these is that the migration algorithms are usually based on simplified physics (high-frequency asymptotics, one-way wave equations, and so on), restricting the approach to dealing with primary reflections only. This motivates us to take a look at the data-domain, where, in principle, we can include multiples by using the full wave-equation to model the data. It should be mentioned that some attempts have been made to use multiples in migration (see, for example, Youn \& Zhou, 2001; Brown \& Guitton, 2005; Jiang et al., 2007).

Data-domain methods come in various shapes and sizes, however, we will focus on waveform inversion. Another class of methods that works in the data-domain is known as traveltime tomography and requires picking of events. Full waveform inversion relies on fitting the measured data. The full wave-equation is used to model the data and the velocity model that minimizes the least-squares (LS) error between measured and simulated data is sought. A well-known problem here is that gradient-based methods fail to converge if the initial velocity model is too far away from the true one. The LS misfit between observed and simulated data does not give a correct model update if seismic events are more than half a wavelength apart. We say that the basin of attraction of the LS functional is small. Numerous studies have been published on the use of the LS approach on the nonlinear problem (Tarantola \& Valette, 1982; Tarantola, 1984; Pratt \& Hicks, 1998, and many others). Because of the absence of low frequencies in the seismic data, the inverse problem is linear in reflectivity (the oscillatory part of the velocity model) and strongly non-linear in the background model (the smooth part of the velocity model). Using a gradient-based method will effectively perform a migration over the initial background (Lailly, 1983) and have great difficulty in updating the background model beyond the depth reached by diving waves (Mora, 1988; Pratt et al., 1996). A method that tries to alleviate this problem is Migration Based Traveltime Tomography (MBTT), proposed by Chavent \& Clément (1993) (see also Plessix et al., 1999; Clément et al., 2001). Here, the iterations consist of two parts: first, a reflectivity for the current velocity model is constructed by one LS iteration; secondly, the data for this reflectivity are modeled, and the LS criterium is used to update the background velocity model. This has been proven to work, however, the 
initial model can still not be too far from the true model. In a way, MBTT can be seen as a data-domain formulation of the MVA methods. The MVA methods measures the amount focusing of the migrated image directly, whereas MBTT generates data for the migrated image and compares this to the measured data. The main difference, apart from the domain the methods work in, is the way the misfit is measured. MVA measures the amount of focusing of a correlation of wavefields and MBTT measures the LS error, which can be seen as an analogue of measuring the stacking-power in the image-domain. In this paper we propose a novel method that measures the amount of focusing of the data correlation. Although the method is intended to be used in a full waveform setting, our goals for this paper are less ambitious. To asses the feasibility of this approach, we restrict ourselves to $1 \mathrm{D}$ velocity models and the convolutional model to generate multiple-free data. The reflectivity and NMO (normal moveout) velocity are parameterized in vertical traveltime. To emulate MBTT we let the reflectivity as a function of vertical traveltime be known. This way, the zero-offset traveltimes are fixed and we can concentrate on updating the background model with the proposed functionals.

The paper is organized as follows. We first explore the properties of the correlation of seismograms and propose a family of functionals to measure the misfit. We give a short overview of image-domain methods and show there are some connections between correlation in the depth-domain, as used in generalized imaging conditions, and correlation in the data-domain. Finally, we present results on synthetic and real data.

\subsection{Data correlation}

Given two seismograms $p(t, h)$ and $q(t, h)$, where $t$ and $h$ denote time and offset respectively, we denote the temporal correlation of these seismograms by

$$
C_{t}[p, q](\Delta t, h)=\int p(t, h) q(t+\Delta t, h) \mathrm{d} t,
$$

the spatial correlation as

$$
C_{h}[p, q](t, \Delta h)=\int p(t, h) q(t, h+\Delta h) \mathrm{d} h,
$$

and the space-time correlation as

$$
C_{t, h}[p, q](\Delta t, \Delta h)=\iint p(t, h) q(t+\Delta t, h+\Delta h) \mathrm{d} t \mathrm{~d} h .
$$

We consider the correlation for finite shifts: $-\Delta t_{\max } \leq \Delta t \leq \Delta t_{\max }$ and $-\Delta h_{\max } \leq$ $\Delta h \leq \Delta h_{\max }$. In Fig. 3.1, we show an example of the auto-correlation of a seismogram and the correlation of two seismograms with different moveout. It is readily observed that if the seismograms are identical (i.e., the velocity model is correct), there is a large peak at $\Delta t=\Delta h=0$. The events at non-zero shifts are caused by 'cross-talk' between events in the seismograms, and are considered noise in the current approach. Note that the temporal correlation suffers most from this cross-talk. 
If the seismograms have different moveout (i.e., the velocity model is not correct) we observe that the energy is not focused in the center. We use this focusing property in the data-domain for velocity analysis. Below, we propose a family of functionals that are based on this property. We show with a few examples that these functionals respond smoothly to velocity perturbations and have a larger basin of attraction than the traditional LS function. Consequently, these functionals should be fit for use with gradient-based optimization scheme.
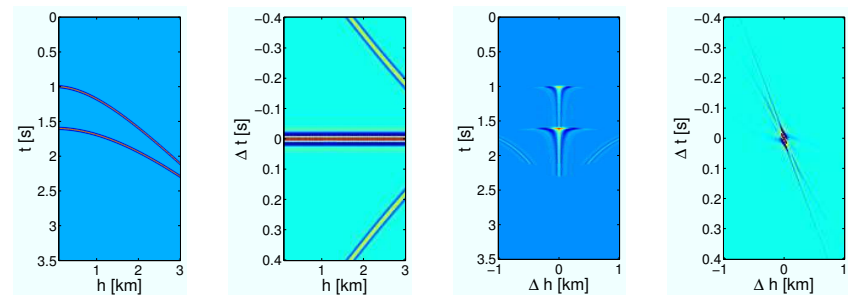

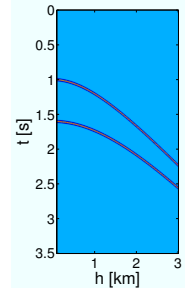

(a)

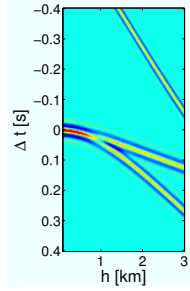

(b)

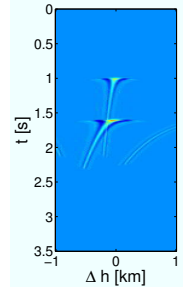

(c)

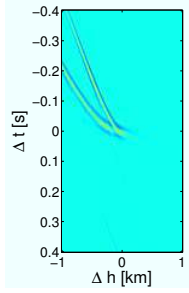

(d)

Figure 3.1. (a) Data, (b) Temporal correlation, (c) spatial correlation and (d) space-time correlation for the correct (top), and a wrong (bottom) velocity model. There is a clear distinction between the focusing of the correlation for the correct and a wrong velocity model. The events that do not focus at zero shift for the correct velocity model are caused by correlations between neighboring events (cross-talk). Note that in (b) and (d), all the events are superimposed around zero shift for all offsets and that in (c) the events all have different zero-offset traveltimes.

\section{Functionals}

We capture the amount of focusing of the correlation functions in a functional. This functional should attain an extremum for the correct velocity model. Luo \& Schuster (1991) use the correlation of first arrivals for traveltime tomography. The functional they proposed to measure the amount of focusing is based on the shifts of the largest peaks in the correlation. This is appropriate if there is only one event in the correlation, but in case of more than one event it is not: Fig. 3.2 shows why. To measure the amount of focusing of the data correlation, we propose to use a weighted norm. The weight should be such that the norm either measures the amount of energy around zero shift (this should be maximized) or the amount of energy away from zero shift (this should be minimized). This approach is also used in some migration-based velocity analysis methods, where the amount of focusing 
of a migrated image is measured in a similar way (Shen et al., 2003). The general form of the class of functionals for the temporal correlation is

$$
\begin{aligned}
J_{t} & =\frac{1}{S_{t}} \iint_{-\Delta t_{\max }}^{\Delta t_{\max }} W(\Delta t)\left(C_{t}[p, q](\Delta t, h)\right)^{2} \mathrm{~d} \Delta t \mathrm{~d} h, \\
S_{t} & =\iint_{-\Delta t_{\max }}^{\Delta t_{\max }}\left(C_{t}[p, q](\Delta t, h)\right)^{2} \mathrm{~d} \Delta t \mathrm{~d} h .
\end{aligned}
$$

where $W(\Delta t)$ is a weighting function, to be specified below. The normalization $S_{t}$ ensures that the functional is not minimized by simply shifting all the events outside of the correlation window. For the spatial and space-time correlation, we define the functionals $J_{h}$ and $J_{t, h}$ in a similar way.

The maximal shift plays a role in reducing the influence of the cross-talk. If the maximal shift is chosen too small, the weighed norm may fail to capture the deviation in focusing of the correlation. If the maximal shift is chosen too large, the cross-talk may dominate over the focusing in the weighted norm.

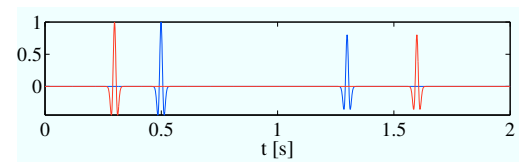

(a)

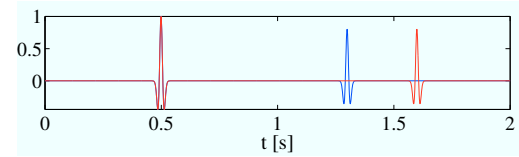

(c)

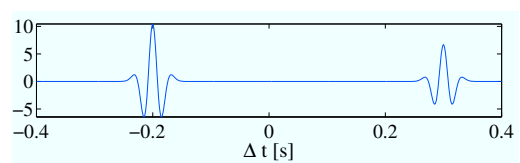

(b)

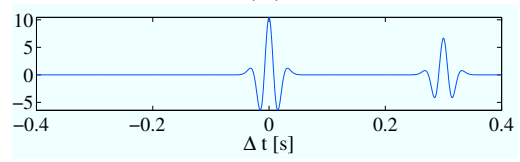

(d)

Figure 3.2. This example shows that the shift of the largest peak in the correlation is not an appropriate measure of similarity. In (a) we see two traces, the 'correct' in blue and the 'wrong' in red. The correlation of the two is depicted in (b). Indeed, the energy is not focused in the center. After correction, the strongest event is in the right place (c) and the correlation looks like (d). Here, we see that the shift of the largest peak is $0 s$ even though the traces are not identical. To properly measure the similarity of the traces, all the peaks in the correlation should be taken into account.

\section{Weighting functions}

We will either measure the amount of energy away from zero shift, or around zero shift. The weighting function we propose to measure the energy away from the center is

$$
W_{1}(\Delta t)=\left(\frac{\Delta t}{\Delta t_{\max }}\right)^{2},
$$

and similarly for the spatial and space-time correlation. This weight will penalize peaks at non-zero shift, and thus measure the amount of energy away from the 
center. The weighted norm can only be zero if the wavelet is a delta pulse and all cross-talk is excluded by choosing an appropriate maximal shift. In the realistic case with a finite-bandwidth wavelet, where we cannot exclude all the cross-talk, the weighted norm will be very small for the correct velocity model.

A weight that measures energy around zero shift, is a Gaussian weighting function

$$
W_{2}(\Delta t)=-e^{-\alpha \Delta t^{2}} .
$$

The minus sign ensures that minimizing the functional will maximize the amount of energy around the center and the parameter $\alpha$ controls the width of the weighting function. For large values of $\alpha$ the exponent decays quickly and the functional only measures the energy in a small region around the center. Peaks that are far away are not taken into account. For small values of $\alpha$, the weighting function is broader and events further away are taken into account. The weighting function should at least be wide enough to capture the event at zero-shift. This means that the width should at least be in the order of the length of the wavelet.
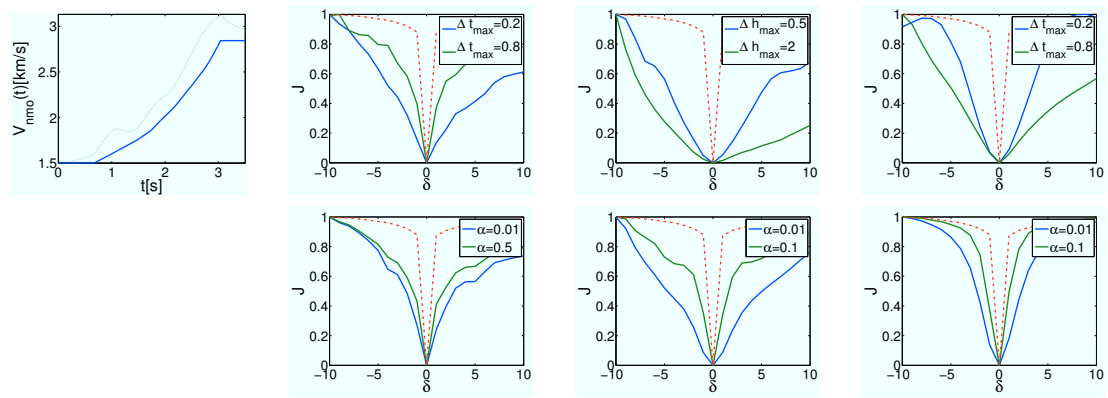

(a)

(b)

(c)

(d)

Figure 3.3. Dependence of the functionals on a perturbation of a the correct velocity model. The functionals are shifted and scaled to allow for visual comparison. The amplitude of the perturbation, $\delta$, is represented on the horizontal axis. The correct velocity model (blue) is depicted in (a), as well as the perturbed model for $\delta=1$ (green). (b) shows the temporal correlation based functional, (c) the spatial correlation and (d) the space-time correlation. The functionals using the quadratic weight are shown on top, and the ones using the Gaussian weight on the bottom. The red dashed lines shows the least-squares functional. The correlationbased functionals are shown for two different values of the width parameter. It seems that for the spatial and space-time correlation, there is a direct relation between the width of the weighting function and the width of the basin of attraction; a larger maximal shift or smaller $\alpha$ results in a wider weighting function and a broader basin of attraction. 
To give an idea of the basin of attraction of the proposed functionals, we show how the functionals respond to a perturbation of the true velocity model in Fig. 3.3. The velocity model (blue) and the perturbed model (green) are depicted in Fig. 3.3 (a). Figures 3.3 (b), (c) and (d) display the temporal, spatial and space-time correlation based functionals using the quadratic weight, defined in Eq. 3.6 (left) and the Gaussian weight, defined in Eq. 3.7 (right). Also depicted is the LS functional in red. For the correlation based functionals the plots are made for different values of the width parameters $\Delta t_{\max }, \Delta h_{\max }$, and $\alpha$. The spatial and space-time correlation based functionals in particular respond smoothly to the perturbation and have a much larger basin of attraction than the LS functional.

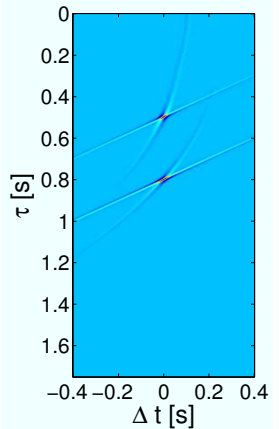

(a)

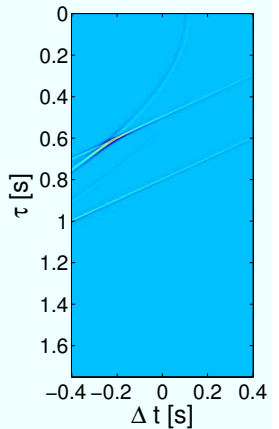

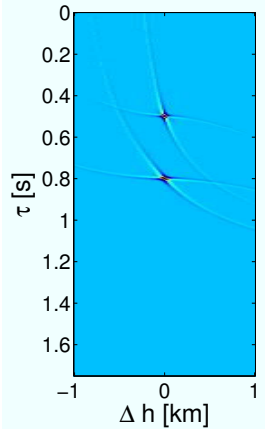

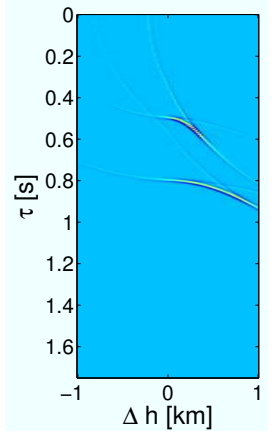

(b)

Figure 3.4. Examples of the generalized image for the data displayed in Fig. 3.1 (a) for the correct (left) and a wrong (right) velocity model. (a) Shows the image at $\Delta h=0$ and (b) at $\Delta t=0$. As with the data correlation, the energy focuses around zero shift for the correct velocity model. Note the absence of crosstalk.

\subsection{Generalized imaging conditions}

The classical imaging condition states that the reflectivity can be recovered by correlating the source and receiver wavefields $u_{s}$ and $u_{r}$ at depth, and extracting the part at zero shift. The generalized imaging condition (Doherty \& Claerbout, 1974; Yilmaz \& Chambers, 1984; MacKay \& Abma, 1992; Rickett \& Sava, 2002; Sava \& Fomel, 2006) allows a finite spatial and temporal shift between the wavefields. The correct velocity model will focus energy in the correlation at zero shift. This approach to velocity analysis is known as migration velocity analysis (MVA). The generalized image is given by

$$
I\left(t_{0}, \Delta t, \Delta h\right)=\iint u_{s}\left(t_{0}, t+\Delta t, h+\Delta h\right) u_{r}\left(t_{0}, t, h\right) \mathrm{d} t \mathrm{~d} h
$$

where $t_{0}$ denotes depth measured in vertical two-way traveltime. For $\Delta t=\Delta h=0$ we obtain the classical imaging condition. With the convolutional model in $1 \mathrm{D}$, the 
wavefields $u_{s}$ and $u_{r}$ are simply time shifted versions of the wavelet and seismogram:

$$
\begin{aligned}
& u_{s}\left(t_{0}, t, h\right)=w\left(t-\frac{1}{2} \tau\left(t_{0}, h\right)\right), \\
& u_{r}\left(t_{0}, t, h\right)=p\left(t+\frac{1}{2} \tau\left(t_{0}, h\right), h\right) .
\end{aligned}
$$

Here, $\tau\left(t_{0}, h\right)$ denotes the two-way traveltime as a function of zero-offset two-way traveltime $t_{0}$ and offset $h$. Thus we can write the generalized image as

$$
I\left(t_{0}, \Delta t, \Delta h\right)=\iint p(t+\Delta t, h+\Delta h) w\left(t-\tau\left(t_{0}, h\right)\right) \mathrm{d} t \mathrm{~d} h
$$

In Fig. 3.4, we show an example of the generalized image for the correct and for a wrong velocity model. As with the data correlation, the energy is focused around zero shift for the correct velocity model. Note that there is no cross-talk present in the generalized image.
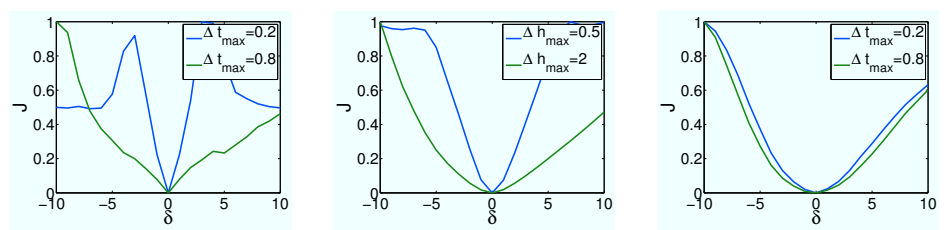

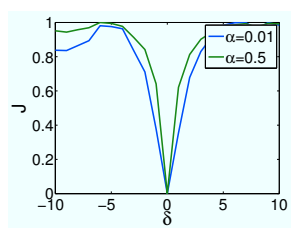

(a)

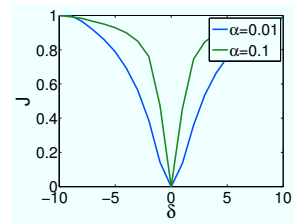

(b)

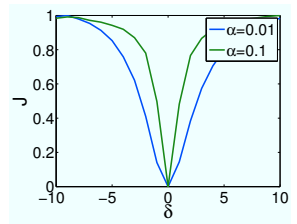

(c)

Figure 3.5. Crossplots for the image-domain functionals. The velocity model and perturbation from Fig. 3.3 are used. The functionals based on the temporal, spatial and space-time correlation are shown in (a), (b), and (c), respectively. The ones on top use the quadratic weight and on the bottom use the Gaussian weight. All the functionals are shown for two different values of the width parameter. The functionals are scaled between 0 and 1 to allow for visual comparison. The functionals show the same behavior as their data-domain analogues.

\section{Functionals}

Shen et al. (2003) propose a functional based on the generalized image and use this in combination with an optimization method to perform automatic MVA (see also Sava \& Biondi, 2004). This approach is akin to Differential Semblance Optimization, proposed by Symes \& Carazzone (1991). The functional is a weighted norm of the generalized image:

$$
J_{h}=\iint \Delta h^{2}\left(I\left(t_{0}, \Delta t=0, \Delta h\right)\right)^{2} \mathrm{~d} t_{0} \mathrm{~d} \Delta h .
$$


Of course, we can also use the functionals proposed before to measure the focusing of the generalized image. Crossplots of the functionals are depicted in Fig. 3.5. These can be compared to the the crossplots of the data-domain functionals. The spatial and space-time correlation based functionals in particular respond smoothly to the perturbation. The functionals show similar behavior as their data-domain analogues.

\subsection{Similarities between data and image-domain focusing}

In this section, we briefly explore the relations between the data and image-domain functionals in the high-frequency limit, under the assumptions of the convolutional model.

Given a reflectivity $r\left(t_{0}\right)$, traveltime $\tau\left(t_{0}, h\right)$, and a wavelet $w(t)$ we model the data as

$$
p(t, h)=\int r\left(\tau_{0}\left(t^{\prime}, h\right)\right) w\left(t-t^{\prime}\right) \mathrm{d} t^{\prime},
$$

where $\tau_{0}(t, h)$ is the inverse of the traveltime such that $\tau\left(\tau_{0}(t, h), h\right)=t$. In the high-frequency limit (the wavelet becomes a delta-pulse) we can write this as

$$
p(t, h)=r\left(\tau_{0}(t, h)\right) .
$$

In the following we will denote the measured or true quantities with a hat $\left(^{\wedge}\right)$ and the simulated quantities without one. In the high-frequency limit we can write the data correlation as

$$
\begin{aligned}
C_{t}[p, \hat{p}](\Delta t, h) & =\int r\left(\tau_{0}(t, h)\right) \hat{r}\left(\hat{\tau_{0}}(t+\Delta t, h)\right) \mathrm{d} t, \\
C_{h}[p, \hat{p}](t, \Delta h) & =\int r\left(\tau_{0}(t, h)\right) \hat{r}\left(\hat{\tau_{0}}(t, h+\Delta h)\right) \mathrm{d} h, \\
C_{t, h}[p, \hat{p}](\Delta t, \Delta h) & =\iint r\left(\tau_{0}(t, h)\right) \hat{r}\left(\hat{\tau_{0}}(t+\Delta t, h+\Delta h)\right) \mathrm{d} t \mathrm{~d} h .
\end{aligned}
$$

The generalized image can be written as

$$
I\left(t_{0}, \Delta t, \Delta h\right)=\int \hat{r}\left(\hat{\tau_{0}}\left(\tau\left(t_{0}, h\right)+\Delta t\right), h+\Delta h\right) \mathrm{d} h .
$$

Note that, for the correct velocity model, $I\left(t_{0}, 0,0\right) \propto \hat{r}\left(t_{0}\right)$.

With a few extra integrals and coordinate transforms we find the following relations

$$
\begin{aligned}
& \int C_{t}[p, \hat{p}](\Delta t, h) \mathrm{d} h=\iint r\left(t_{0}\right) \hat{r}\left(\hat{\tau_{0}}\left(\tau\left(t_{0}, h\right)+\Delta t, h\right)\right) s\left(t_{0}, h\right) \mathrm{d} t_{0} \mathrm{~d} h, \\
& \int C_{h}[p, \hat{p}](t, \Delta h) \mathrm{d} t=\iint r\left(t_{0}\right) \hat{r}\left(\hat{\tau_{0}}\left(\tau\left(t_{0}, h\right), h+\Delta h\right)\right) s\left(t_{0}, h\right) \mathrm{d} h \mathrm{~d} t_{0}, \\
& C_{t, h}[p, \hat{p}](\Delta t, \Delta h)= \\
& \iint r\left(t_{0}\right) \hat{r}\left(\hat{\tau_{0}}\left(\tau\left(t_{0}, h\right)+\Delta t, h+\Delta h\right)\right) s\left(t_{0}, h\right) \mathrm{d} t_{0} \mathrm{~d} h .
\end{aligned}
$$


where $s\left(t_{0}\right)=\frac{\partial \tau}{\partial t_{0}}\left(t_{0}, h\right)$ is a stretch factor due to the coordinate change from time to depth. If $s\left(t_{0}, h\right) \approx 1$ - this is also the range for which the convolutional model is valid (Symes, 1999), typically offsets larger than 1000t are blanked out - we find the following relations between the data correlation and the generalized image

$$
\begin{aligned}
\int C_{t}[p, \hat{p}](\Delta t, h) \mathrm{d} h & \sim \int r\left(t_{0}\right) I\left(t_{0}, \Delta t, \Delta h=0\right) \mathrm{d} t_{0}, \\
\int C_{h}[p, \hat{p}](t, \Delta h) \mathrm{d} t & \sim \int r\left(t_{0}\right) I\left(t_{0}, \Delta t=0, \Delta h\right) \mathrm{d} t_{0}, \\
C_{t, h}[p, \hat{p}](\Delta t, \Delta h) & \sim \int r\left(t_{0}\right) I\left(t_{0}, \Delta t, \Delta h\right) \mathrm{d} t_{0} .
\end{aligned}
$$

This shows that, the space-time correlation is a weighted integral of the generalized image over depth.

\section{Functionals}

With the relations derived above we can express the data-domain functional as (leaving out the normalization factor $S$ for the moment)

$$
\begin{aligned}
J_{t, h}^{\text {data }} & =\iint W(\Delta t, \Delta h)\left(C_{t, h}[p, \hat{p}](\Delta t, \Delta h)\right)^{2} \mathrm{~d} \Delta t \mathrm{~d} \Delta h \\
& =\iint W(\Delta t, \Delta h)\left(\int r\left(t_{0}\right) I\left(t_{0}, \Delta t, \Delta h\right) \mathrm{d} t_{0}\right)^{2} \mathrm{~d} \Delta t \mathrm{~d} \Delta h .
\end{aligned}
$$

The corresponding image-domain functional is given by

$$
J_{t, h}^{\text {image }}=\iiint W(\Delta t, \Delta h)\left(I\left(t_{0}, \Delta t, \Delta h\right)\right)^{2} \mathrm{~d} \Delta t \mathrm{~d} \Delta h \mathrm{~d} t_{0} .
$$

The main difference between the data and image-domain functionals here is the order in which the weighting and integration over depth is carried out. Also, the image is weighted with the reflectivity in the data-domain functional. Despite the apparent difference between the image- and data-domain approaches, it seems that the functionals exhibit similar behavior when we consider the crossplots in Figs 3.3 and 3.5.

\subsection{Tests with synthetic data}

Here, we present results for velocity analysis on synthetic data for two $1 \mathrm{D}$ velocity models. The data are generated with the convolutional model. The experiments are intended as a first test, to see if the method proposed here is promising. We will give a brief overview of the experimental setup (generation of synthetic data, optimization method), followed by the results of these experiments. 


\section{Synthetic data}

The synthetic data were modeled with the convolutional model, see Eq. 3.13. The traveltimes are given by

$$
\tau\left(t_{0}, h\right)=\sqrt{t_{0}^{2}+\left(h / v_{\mathrm{nmo}}\left(t_{0}\right)\right)^{2}},
$$

where $t_{0}$ denotes depth in vertical traveltime and $v_{\text {nmo }}\left(t_{0}\right)$ is the NMO velocity. The reflectivity is a spike train and as wavelet we used a Ricker wavelet with a peak frequency of $30 \mathrm{~Hz}$.

For the experiments, we have used two velocity models, depicted in Fig. 3.6. We started the optimization from a constant initial velocity model. The synthetic data were generated with the 'true' reflectivity, which basically means that the zero-offset traveltimes were fixed and we only had to correct for a wrong moveout.

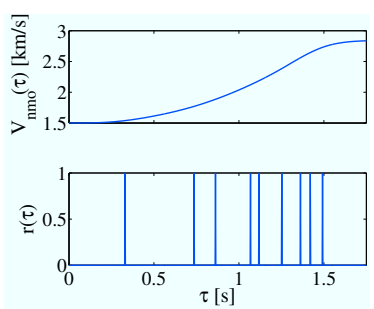

(a)
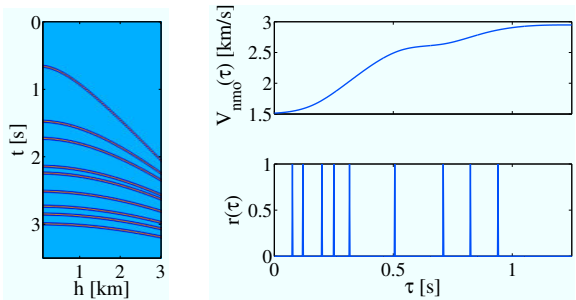

(b)

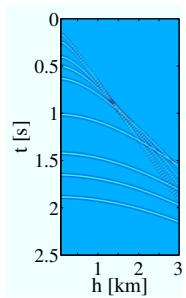

Figure 3.6. (a,b) $1 D$ velocity model, reflectivity and corresponding data used in the experiments.

\section{Optimization method}

We represent the NMO velocity on a cubic-spline grid and use the values at the nodes as our model parameters. For the optimization we use a BFGS (BroydenFletcher-Goldfarb-Shanno) method with the exact gradient. This gradient is derived in Appendix A. It proved desirable to use smoothness constraint to avoid unnecessary peaks in the solutions. For an overview of the BFGS method and Tikhonov regularization we refer to Vogel (2002).

\section{Results}

Figures 3.7-3.10 show the results of the experiments. For each experiment, we depict the initial, final, and true velocity model. Obviously, the inversion was successful if the true velocity model was recovered. In some instances, we found perfectly matching simulated data for somewhat different velocity models. This is caused by the null-space of the problem and can be dealt with by regularization. We chose to use a Tikhonov regularization that penalizes large variations in the velocity model. The data correlation for the initial and final velocity models are displayed to show 
the difference between the focusing of the correlation.

Figures 3.7 and 3.8 display the results for the functional based on the temporal correlation with the Gaussian weighting function. Although the final velocity models are not identical to the true ones, they do reproduce the data perfectly. The correlations for the initial velocity models show a deviation in the focusing (Figs 3.7(b) and 3.8(b), left). For the final velocity model, the correlation is focused (same Figs, right). For the more complicated velocity model (Fig. 3.8(a)), the focusing is less distinct due to the cross-talk. Nevertheless, the functional was able to capture the focusing.

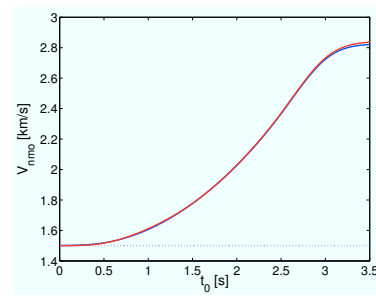

(a)

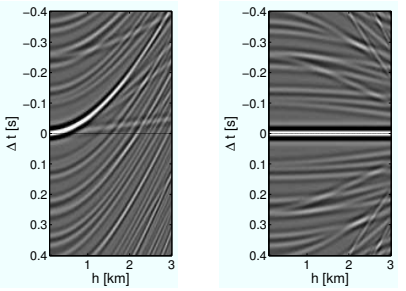

(b)

Figure 3.7. Results obtained for the correlation-based functional with the Gaussian weighting function. (a) Correct (blue), initial (dots), and final (red) velocity model. (b) Initial (left) and final (right) correlation. The iterations converged to the true velocity model.

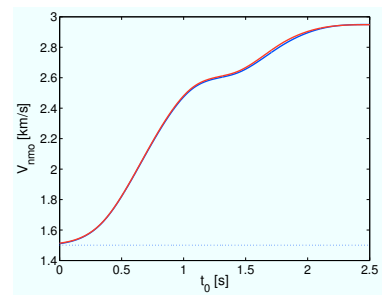

(a)

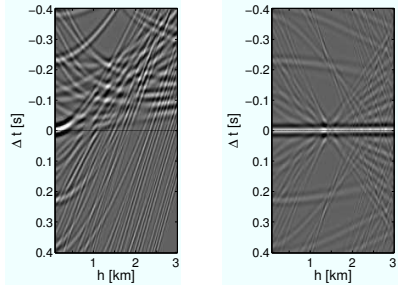

(b)

Figure 3.8. Same as figure 3.7 but for a different velocity model.

In Figs 3.9 and 3.10, the results for the functional based on the spatial correlation with the Gaussian weighting function are shown. As in the previous results, the initial and true velocity models are not identical, but the data are. Again, this is caused by a lack of reflectors in the regions where the velocity models disagree. In the correlation (Figs 3.9(b) and 3.10(b), left) we see that the events are separated in traveltime, hence the focus of every event can be measured independently. It also 
shows that the deviation in the focusing increases with time. This agrees with the error in the initial velocity model, which also increases with time.

We present only a few of several successful experiments. The functionals based on the space-time correlation $\left(J_{t, h}\right)$ also proved successful on the test problems. Overall, the Gaussian weighting function worked best on our two test problems. This is probably because this weighting function is better able to cope with the cross-talk. Combining the spatial and temporal correlation in one functional by adding $J_{t}$ and $J_{h}$ is another possibility we did not consider. We have not compared the methods in terms of convergence speed, since computational cost is not an issue with the convolutional model.

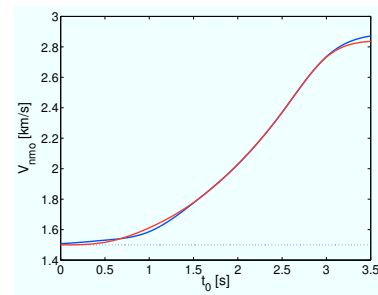

(a)
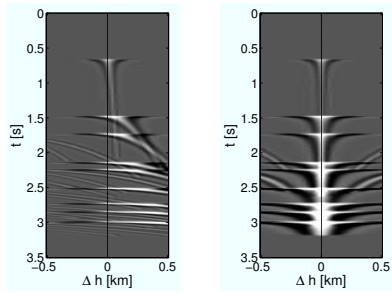

(b)

Figure 3.9. Same as figure 3.7 but for the space correlation.

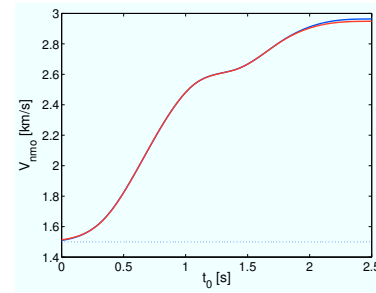

(a)
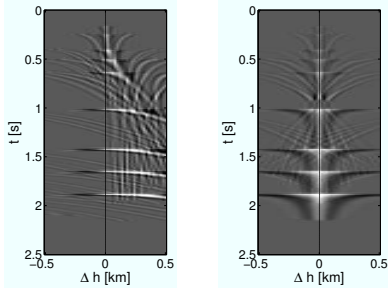

(b)

Figure 3.10. Same as figure 3.9 but for a different velocity model.

\subsection{Test with real data}

We also tested the proposed functionals on real data. The synthetic data are again modeled with the convolutional model. The reflectivity for the synthetic data is taken to be the first trace of the observed data. The wavelet used is a Ricker wavelet with a peak frequency of $30 \mathrm{~Hz}$. The real data were slightly processed in an attempt to remove the surface related multiples.

In Figs 3.11-3.13 the results of an inversion using the spatial correlation and the Gaussian weighting function are depicted. The initial and final velocity model are shown in Fig. 3.11(b). Also, the NMO-corrected data for the initial and final velocity model are shown in Fig. 3.11(a), and the flatness of the events indicates 
that the final velocity model is indeed the 'correct' NMO velocity. The final velocity model focuses both the data (Fig. 3.12) and image (Fig. 3.13) correlation, which suggests an equivalence between the focusing in both domains.

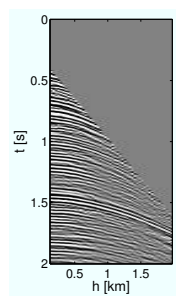

(a)

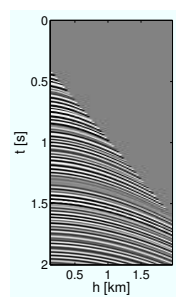

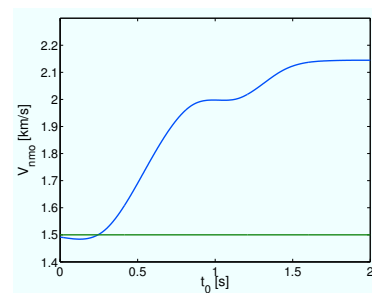

(b)

Figure 3.11. Real data example. The simulated data are generated with the convolutional model, using the first trace of the data as reflectivity and a Ricker wavelet with a peak frequency of $30 \mathrm{~Hz}$. (a) Shows the real data (left) and the synthetic data (right) for the final velocity model, which resulted from using the functional based on the spatial correlation with the Gaussian weighting function. (b) shows the initial (green) and final (blue) velocity models.
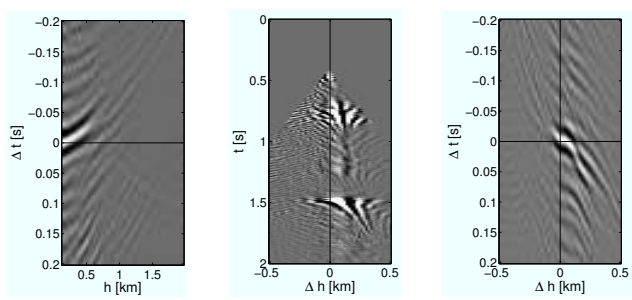

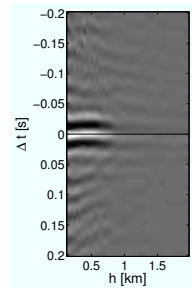

(a)

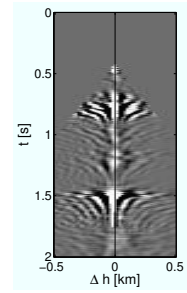

(b)

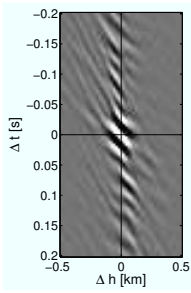

(c)

Figure 3.12. Data correlation for the real data example from Fig. 3.11. The temporal (a), spatial (b), and space-time correlation (c) is depicted for the initial (top) and final (bottom) velocity models. Note that, although we used the functional based on the spatial correlation, the final velocity model focuses all three. 


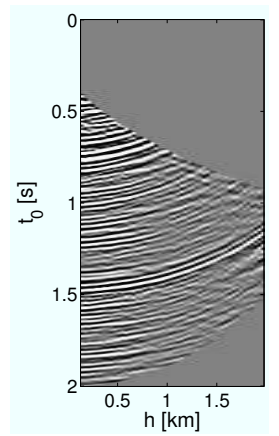

(a)

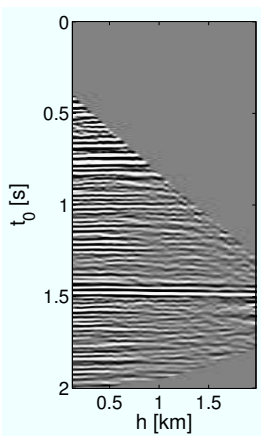

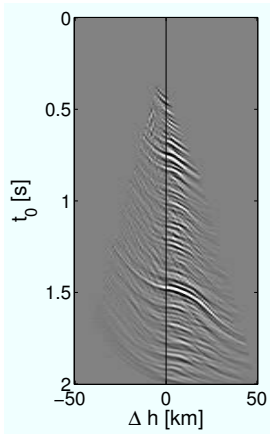

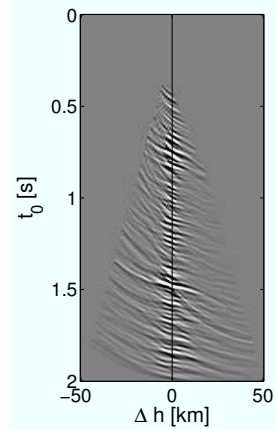

(b)

Figure 3.13. Focussing in the image-domain for the real data example from Fig. 3.11. (a) shows the NMO corrected data for the initial (left) and final (right) velocity model. The events are flattened by the final velocity model, which indicates that this is indeed the correct $N M O$ velocity. In (b) the generalized image is shown for $\Delta t=0$, again for the initial (left) and final (right) velocity models. The final velocity model focuses the generalized image. This suggests an equivalence between the focusing in the the data- and image-domains.

\subsection{Conclusions and discussion}

We explored the use of correlation of seismograms for velocity analysis for $1 \mathrm{D}$ velocity models. The synthetic seismograms were generated with the convolutional model. The examples showed that the correlation focuses at zero shift for the correct velocity model and responds to errors in the velocity model by de-focusing. To measure the amount of focusing, we proposed a family of functionals. These functionals are weighted norms of the correlation function. In the correlation, peaks that do not focus at zero shift are also present. The functionals have a width parameter to suppress this cross-talk. From the plots of the data-focusing functionals it seems that the time correlation suffers most from the cross-talk. The Gaussian weighting function deals with the cross-talk successfully. Compared to the LeastSquares functional, most of the weighted norms have a large basin of attraction and respond smoothly to a perturbation of the true velocity model.

We showed that under the assumptions of the convolutional model, there is a strong connection between the functionals based on the space-time correlation in the depth and the data-domain, the main difference being the order in which the weighting and integration over depth is carried out. In the data focusing approach the weighting is applied to the squared sum of the images, while in the data focusing approach the weighting is applied to the sum of the squared images. Overall, the data and image-domain functionals respond similarly to a perturbation.

To test the functionals proposed here, we used a gradient-based optimization scheme to solve for the true velocity model, starting from a constant initial model. We have shown only a few experiments, but all the functionals yielded good results. Espe- 
cially the Gaussian weighting function proved successful, whereas the quadratic weighting function showed more sensitivity to the width parameter.

The real data example showed that the velocity model resulting from optimizing the data-focusing also focused the generalized image and flattened the events after NMO correction. This suggests an equivalence between the data and image-domain methods.

It remains to be seen if the method can be successfully extended beyond the convolutional model for 1D models. A next step will be to test the proposed functionals in a full waveform setting. A point of special interest is whether this method will succeed when multiples are present, because this is where the image-domain methods tend to break down.

\subsection{Appendix: Gradient}

We use the exact gradients for the optimization. We explicitly derive the gradient for the functional based on the temporal correlation using the adjoint state technique (see Plessix, 2006, for example). The gradients for the spatial and space-time correlation can be derived in the same way. Some of the arguments of the functions have been left out for notational clarity.

We represent the velocity model with parameters $\left\{v_{0}, v_{1}, \ldots, v_{N-1}\right\}$ on an equidistant grid $\left\{t_{0}, t_{1}, \ldots, t_{N-1}\right\}$ of spline nodes. Given these parameters the NMO velocity is represented by

$$
v_{\mathrm{nmo}}(t)=\sum_{i=0}^{N-1} v_{i} \psi\left(t-t_{i}\right)
$$

where $\psi(t)$ is a cubic spline. We use the convolutional model to generate the data, so the simulated seismogram can be written as

$$
\begin{aligned}
& p(t, h)=\int w\left(t-t^{\prime}\right) r\left(\tau_{0}\left(t^{\prime}, h\right)\right) \mathrm{d} t^{\prime}= \\
& \int w\left(t-\tau\left(t_{0}, h\right)\right) r\left(t_{0}\right) s\left(t_{0}, h\right) \mathrm{d} t_{0},
\end{aligned}
$$

where $w(t)$ is the wavelet, $r(t)$ is the reflectivity, in our case a spike train, and $\tau_{0}(t, h)$ is the inverse of the traveltime:

$$
\tau\left(t_{0}, h\right)=\sqrt{t_{0}+\left(h / v_{\mathrm{nmo}}(t)\right)^{2}}
$$

Here $h$ denotes offset and $t_{0}$ is depth measured in vertical two-way traveltime. We assume that the stretch factor $s\left(t_{0}, h\right)=\frac{\partial \tau}{\partial t_{0}}\left(t_{0}, h\right)$ can be neglected for the rest of the derivation. In fact, the convolutional model is only valid for $s\left(t_{0}, h\right) \approx 1$ (Symes, 1999). Typically, offsets larger than $1000 t$ are blanked out. The functional is given 
by

$$
\begin{aligned}
J_{t} & =\frac{1}{S_{t}} \iint W(\Delta t)\left(C_{t}[p, q](\Delta t, h)\right)^{2} \mathrm{~d} \Delta t \mathrm{~d} h \\
+ & \beta \sum_{i}\left(v_{i+1}-v_{i}\right)^{2}, \\
S_{t} & =\iint\left(C_{t}[p, q](\Delta t, h)\right)^{2} \mathrm{~d} \Delta \mathrm{d} \Delta h t
\end{aligned}
$$

The second term in the functional is the Tikhonov regularization, which penalizes large variations in the velocity model. The parameter $\beta$ controls the amount of regularization applied. To avoid a large penalty at the edges of the domain we let $v_{-1}=v_{0}$ and $v_{N}=v_{N-1}$.

To find an expression for the gradient $\frac{\partial J_{t}}{\partial v_{i}}$, we consider the Lagrangian:

$$
\begin{aligned}
& \mathcal{L}=\frac{1}{S_{t}} \iint W(\Delta t)\left(C_{t}[p, q](\Delta t, h)\right)^{2} \mathrm{~d} \Delta t \mathrm{~d} h+\beta \sum_{i}\left(v_{i+1}-v_{i}\right)^{2} \\
& +\iint \mu_{1}(\Delta t, h)\left(C_{t}[p, q]-\int p(t, h) q(t+\Delta t, h) \mathrm{d} t\right) \mathrm{d} \Delta t \mathrm{~d} h \\
& +\iint \mu_{2}(t, h)\left(p(t, h)-\int w\left(t-\tau\left(t_{0}, h\right)\right) r\left(t_{0}\right) \mathrm{d} t_{0}\right) \mathrm{d} t \mathrm{~d} h \\
& +\iint \mu_{3}\left(t_{0}, h\right)\left(\tau\left(t_{0}, h\right)-\sqrt{t_{0}^{2}+\left(h / v_{\mathrm{nmo}}\left(t_{0}\right)\right)^{2}}\right) \mathrm{d} t_{0} \mathrm{~d} h \\
& +\int \mu_{4}\left(t_{0}\right)\left(v_{\mathrm{nmo}}\left(t_{0}\right)-\sum_{i=0}^{N-1} v_{i} \psi\left(t_{0}-t_{i}\right)\right) \mathrm{d} t_{0},
\end{aligned}
$$

where $\mu_{1} \ldots \mu_{4}$ are the Lagrange multipliers. The gradients of the Lagrangian w.r.t. the state variables $C_{t}, p, \tau$ and $v_{\text {nmo }}$ are given by

$$
\begin{aligned}
\frac{\partial \mathcal{L}}{\partial C_{t}} & =\frac{2}{S_{t}}\left(W-J_{t}\right) C_{t}[p, q](\Delta t, h)+\mu_{1}(\Delta t, h), \\
\frac{\partial \mathcal{L}}{\partial p} & =-\int \mu_{1}(\Delta t, h) p(t+\Delta t, h) \mathrm{d} \Delta t+\mu_{2}(t, h), \\
\frac{\partial \mathcal{L}}{\partial \tau} & =+\int \mu_{2}(t, h) \dot{w}\left(t-\tau\left(t_{0}, h\right)\right) r\left(t_{0}\right) \mathrm{d} t \\
& +\mu_{3}\left(t_{0}, h\right), \\
\frac{\partial \mathcal{L}}{\partial v_{\mathrm{nmo}}} & =+\int \mu_{3}\left(t_{0}, h\right) \frac{h^{2} v_{\mathrm{nmo}}^{-3}}{\tau\left(t_{0}, h\right)} \mathrm{d} h+\mu_{4}\left(t_{0}\right) .
\end{aligned}
$$

Here $\dot{w}(t)$ we denotes the temporal derivative of $w(t)$.

At a stationary point, $\nabla \mathcal{L}=0$. Using this, we can eliminate the multipliers by successive substitution. This yields an expression for $\mu_{4}$ which can be used to 
determine the gradient of the functional

$$
\begin{aligned}
& \frac{\partial J_{t}}{\partial v_{i}}=\frac{\partial \mathcal{L}}{\partial v_{i}}-\int \mu_{4}\left(t_{0}\right) \psi\left(t_{0}-t_{i}\right) \mathrm{d} t_{0}-2 \beta\left(v_{i+1}-2 v_{i}+v_{i-1}\right)= \\
& \frac{2}{S_{t}} \int \mathrm{d} t_{0} r\left(t_{0}\right) \psi\left(t_{0}-t_{i}\right) \\
& \times\left(\iint C_{t}\left[\left(W-J_{t}\right) C_{t}[p, q], q\right] \dot{w}\left(\left(t-\tau\left(t_{0}, h\right)\right) \frac{h^{2} v_{\text {nmo }}^{-3}}{\tau\left(t_{0}, h\right)} \mathrm{d} t \mathrm{~d} h\right)\right.
\end{aligned}
$$

To calculate the gradient we: (i) do weighted correlation of the data correlation and the observed data, (ii) correlate this with a wavelet (the derivative of the original wavelet), (iii) stack the NMO corrected result and (iv) project this back onto the spline basis. 


\title{
4 \\ A comparison of seismic velocity analysis methods for layered acoustics
}

T. van Leeuwen and W.A. Mulder. 2010. Inverse problems, 26(1):015008

\begin{abstract}
In seismic imaging, one tries to infer the medium properties of the subsurface from seismic reflection data. These data are the result of an active source experiment, where an explosive source and an array of receivers are placed at the surface. Due to the absence of low frequencies in the data, the corresponding inverse problem is strongly non-linear in the slowly varying component of the velocity. The least-squares misfit functional typically exhibits local minima and has a small basin of attraction. The usual approach of fitting the data in a least-squares sense by employing a gradient-based optimisation method will therefore most likely result in a wrong velocity model. In the geophysical community, this problem has long been recognized and alternative formulations of the inverse problem have been developed. We review several of these formulations and analyze the sensitivity to the error in the smooth velocity component. This analysis is carried out for laterally homogeneous velocities using an asymptotic solution of the wave equation. The analysis suggests that formulations which are geared towards fitting the phases of the data, rather than the amplitudes, have smooth corresponding misfit functionals with a large basin of attraction.
\end{abstract}




\subsection{Introduction}

In seismic imaging, one tries to infer the medium properties of the subsurface from seismic reflection data. These data are the result of an active source experiment, where an explosive source and an array of receivers are placed at or, in the case of a marine experiment, just below the surface. In the constant-density acoustic approximation, the wave propagation is governed by a scalar wave equation and the medium parameter to be recovered is the sound speed in the subsurface. The corresponding inverse problem can be formulated as a PDE-constrained least-squares (LS) problem. The sheer size of a typical problem in seismic exploration dictates that the resulting optimisation problem is solved using a gradient-based optimisation algorithm. This approach to seismic imaging was pioneered by Tarantola (1984). However, it soon became apparent that the band-limited nature of the data causes local minima to exist in the least-squares objective functional. Hence, a very good initial guess of the subsurface velocity model is needed to converge to the global minimum.

Due to the absence of low frequencies in the source, the reflections in the data are caused by discontinuities in the velocity. This allows us to separate scales, and split the velocity into a smooth 'background' component and an oscillatory component, 'the reflectivity'. Ignoring multiple scattering, the data are now linear in the reflectivity. Under these assumptions, the inverse problem has become separable: the background velocity appears as a non-linear parameter, while the reflectivity appears as a linear parameter. A straightforward approach to solve such a separable LS problem, is to use a variable projection algorithm Golub \& Pereyra (1973). The basic idea is to resolve the reflectivity via the normal equations. In the literature this is often referred to as migration Bednar (2005). Effectively, the data are projected onto the range of the modelling operator, and the LS difference between the data and their projection is minimized. This approach was applied to seismic imaging by Chavent et al. (1994). While this partly mitigates the nonlinearity of the inverse problem, the resulting misfit functional still has a small basin of attraction. This is because the range of the modelling operator changes very rapidly as the background velocity is perturbed. This causes the data and their projection for a perturbed velocity to be almost orthogonal.

To circumvent the problem of local minima and a small basin of attraction, a number of approaches exist that recast the optimisation problem as a tomographic problem. This is referred to as 'velocity analysis' in the literature Claerbout (1984); Yilmaz (1987). We discern two distinct classes of such methods. The first exploits the redundancy in the data to introduce a redundant coordinate in the reflectivity estimate. For the correct velocity model, the reflectivity estimate should not depend on this redundant coordinate, and this serves as a criterion to obtain the correct background velocity. These methods have been well-established Symes (1991); Chauris \& Noble (2001); Mulder \& ten Kroode (2002); Shen \& Symes (2008) and a general theoretical framework exists that encompasses these methods. The main result here is that an exact annihilator can be constructed Stolk \& de Hoop (2006); de Hoop et al. (2006). This means that an exact criterion can be derived to measure the velocity error. It can be shown that the corresponding annihilators will indeed lead to 
misfit functionals that behave smoothly as a function of the velocity error Stolk \& Symes (2003). For an extensive overview we refer to Symes (2008a) and references cited therein.

The second class of velocity analysis methods exploits the redundancy in the data directly. These methods are akin to the LS data-fitting approach, described above, except that they use a misfit criterion different from the LS error Luo \& Schuster (1991); van Leeuwen \& Mulder (2008a). In this paper we review several velocity analysis methods in the context of laterally invariant velocities. First, we derive an asymptotic solution to the wave-equation for laterally invariant velocities and define the modelling operator. This type of modelling is known as 'convolutional' modelling and is well-established. Nevertheless, we include an outline of the derivation for the sake of completeness. We present an example of seismic data modelled for a simple velocity model and compare it with the solution of the wave-equation modelled with finite differences. In section 4 , we briefly discuss the linearized inverse problem. Several velocity analysis methods are discussed in sections 5 and 6 . Although a quite general framework exists that encompasses some of these methods, we present only the very basics, providing an intuitive understanding of the general principles. Using stationary phase analysis, we examine the sensitivity to velocity errors. We include scans of the objective functionals, which show their typical behavior. In section 7 we discuss the apparent relation between two particular methods. Finally, we present an example of several methods on synthetic data in section 8 and conclude the paper in section 9 .

\subsection{Notation and preliminaries}

- $\mathbf{x}$ denotes a vector in $\mathbb{R}^{3}$, individual components are denoted by $x_{(i)}$. For variables in $\mathbb{R}$, italics will be used.

- The complex conjugate of a scalar as well as the complex conjugate transpose, or adjoint, of an operator will be denoted by *

- $\langle\cdot, \cdot\rangle$ denotes the inner product. For vectors, $\langle\mathbf{x}, \mathbf{y}\rangle=\sum_{i} x_{(i)}^{*} y_{(i)}$, for functions, $\langle f, g\rangle=\int \mathrm{d} x f^{*}(x) g(x)$.

- $\|\cdot\|$ denotes the $L_{2}$ norm, defined for vectors and functions in the usual way via the inner product $\|\cdot\|=\sqrt{\langle\cdot, \cdot\rangle}$.

- [.] denotes functional dependence. For instance, $J[f]=\|f\|$ and $C[f, g](x)=$ $\int \mathrm{d} x^{\prime} f\left(x^{\prime}\right) g\left(x-x^{\prime}\right)$.

- $\sim$ denotes an asymptotic relation. This indicates that we neglect smooth errors, as opposed to $\approx$ which indicates neglect of small errors.

An integral of the form

$$
I=\int \mathrm{d} x f(x) \exp [\imath \omega \phi(x)]
$$


can be approximated for large $\omega$ by

$$
I \sim\left(1+\imath \operatorname{sign}\left(\phi^{\prime \prime}\left(x_{0}\right)\right)\right) \sqrt{\frac{\pi}{\omega\left|\phi^{\prime \prime}\left(x_{0}\right)\right|}} f\left(x_{0}\right) \exp \left[\imath \omega \phi\left(x_{0}\right)\right],
$$

where $x_{0}$ is called the stationary point where $\phi^{\prime}\left(x_{0}\right)=0$. A derivation can be found in may textbooks on asymptotic analysis (Bleistein \& Handelsman, 1975, for example). We will deploy this stationary-phase approximation throughout the paper. Since we are mainly interested in the phase behavior, we will neglect the amplitude factor $(1 \pm \imath) \sqrt{\pi /\left|\phi^{\prime \prime}\right|}$ most of the time.

\subsection{Modelling}

We use the so-called convolutional model with hyperbolic traveltimes to compute the seismic data. This type of modelling is valid for layered media at small sourcereceiver distances (offsets). Although this type of modelling is well-established, we present a derivation for the sake of completeness.

The seismic data sample the wavefield at some receiver position $\mathbf{x}_{r}:\left.u\left(\omega, \mathbf{x}, \mathbf{x}_{s}\right)\right|_{\mathbf{x}=\mathbf{x}_{r}}$. Here, $u$ is assumed to obey the scalar Helmholtz equation

$$
L[c] u\left(\omega, \mathbf{x}, \mathbf{x}_{s}\right)=f(\omega) \delta\left(\mathbf{x}-\mathbf{x}_{s}\right),
$$

where $L[c]=(\omega / c)^{2}+\nabla^{2}, \mathbf{x}_{s}$ and $\mathbf{x}_{r}$ denote the source and receiver positions, $\mathbf{x}$ denotes the subsurface coordinate, $c(\mathbf{x})$ gives the soundspeed in the earth, and $f$ is the source signature. It is common practise in seismic imaging to use a Born approximation and split the squared slowness into a smooth ('background velocity') and an oscillatory ('reflectivity') part: $c(\mathbf{x})^{-2}=c_{0}(\mathbf{x})^{-2}+r(\mathbf{x})$. The equations for the incident and scattered wavefield read:

$$
\begin{aligned}
& L\left[c_{0}\right] u_{0}\left(\omega, \mathbf{x}, \mathbf{x}_{s}\right)=f(\omega) \delta\left(\mathbf{x}-\mathbf{x}_{s}\right), \\
& L\left[c_{0}\right] u_{1}\left(\omega, \mathbf{x}, \mathbf{x}_{s}\right)=-\omega^{2} u_{0}\left(\omega, \mathbf{x}, \mathbf{x}_{s}\right) r(\mathbf{x}) .
\end{aligned}
$$

In terms of the Green's function for the Helmholtz equation, the incident and scattered wavefields are given by

$$
\begin{aligned}
& u_{0}\left(\omega, \mathbf{x}, \mathbf{x}_{s}\right)=f(\omega) G\left(\omega, \mathbf{x}, \mathbf{x}_{s}\right), \\
& u_{1}\left(\omega, \mathbf{x}, \mathbf{x}_{s}\right)=-\omega^{2} \int \mathrm{d} \mathbf{x}^{\prime} r\left(\mathbf{x}^{\prime}\right) u_{0}\left(\omega, \mathbf{x}^{\prime}, \mathbf{x}_{s}\right) G\left(\omega, \mathbf{x}, \mathbf{x}^{\prime}\right) .
\end{aligned}
$$

For the Green's function we use a high-frequency (WKBJ) approximation

$$
G\left(\omega, \mathbf{x}, \mathbf{x}^{\prime}\right) \sim A\left(\mathbf{x}, \mathbf{x}^{\prime}\right) \exp \left[\imath \omega T\left(\mathbf{x}, \mathbf{x}^{\prime}\right)\right]
$$

where $A$ is the amplitude factor which obeys a transport equation and $T$ is the traveltime which obeys an eikonal equation:

$$
\begin{aligned}
\nabla T \cdot \nabla T & =c(\mathbf{x})^{-2}, \\
\nabla \cdot\left(A^{2} \nabla T\right) & =0 .
\end{aligned}
$$


Assuming that the data sample only the scattered field $u_{1}$, we define a linear operator that maps the reflectivity $r$ to data $p$ :

$$
p\left(\omega, \mathbf{x}_{s}, \mathbf{x}_{r}\right)=F\left[c_{0}\right] r=u_{1}\left(\omega, \mathbf{x}_{s}, \mathbf{x}_{r}\right) .
$$

\subsubsection{Laterally invariant velocity}

To simplify the subsequent analysis we make the following assumptions

- the velocity is laterally invariant;

- there is one source at the origin and the receivers are positioned at the surface, i.e., $x_{r,(3)}=0$;

- we are interested in relatively small source-receiver distances (offsets);

- we can neglect smoothly varying amplitude factors because we are interested in the singular behavior of the data.

Under these assumptions, the data, $p(\omega, h)=u_{1}\left(\omega, \mathbf{x}_{s}, \mathbf{x}_{r}\right)$, depend only on halfoffset $h=\frac{1}{2}\left\|\mathbf{x}_{r}-\mathbf{x}_{s}\right\|$ and frequency. Using a stationary-phase approximation to get rid of the integrals over the lateral coordinates and taking into account that $x_{r,(3)}=x_{s,(3)}=0$, we can approximate eq. (4.11) by

$$
p(\omega, h)=F\left[c_{0}\right] r \sim-\omega f(\omega) \int \mathrm{d} z r(z) \exp \left[\imath \omega \tau\left[c_{0}\right](z, h)\right],
$$

where

$$
\tau\left[c_{0}\right](z, h)=2 \int_{0}^{z} \mathrm{~d} \zeta c_{0}(\zeta)^{-1}+h^{2}\left(\int_{0}^{z} \mathrm{~d} \zeta c_{0}(\zeta)^{-1}\right)^{-1} m(z)
$$

denotes the two-way traveltime and $m$ is the effective squared slowness:

$$
m(z)=\frac{\int_{0}^{z} \mathrm{~d} \zeta c_{0}(\zeta)^{-1}}{\int_{0}^{z} \mathrm{~d} \zeta c_{0}(\zeta)}
$$

This approximation to the two-way traveltime relates to the solution of the eikonal equation as $T(0, \mathbf{x})=\frac{1}{2} \tau\left(x_{3}, \frac{1}{2} \sqrt{x_{1}^{2}+x_{2}^{2}}\right)+\mathcal{O}\left(h^{4}\right)$. For a formal derivation we refer to Dix (1955); Symes (1999). The quantity $1 / \sqrt{m}$ is sometimes referred to as the root-mean-square (RMS) velocity. The traveltime $\tau(z, h)$ is called the normal moveout (NMO) traveltime.

Next, we parameterize depth by the vertical two-way traveltime $t_{0}(z)=2 \int_{0}^{z} \mathrm{~d} \zeta c_{0}(\zeta)^{-1}$ and define the convolutional modelling operator as

$$
\tilde{F}[\tilde{m}] \tilde{r}=-\omega f(\omega) \int \mathrm{d} t_{0} \tilde{r}\left(t_{0}\right) \exp \left[\imath \omega \tilde{\tau}[\tilde{m}]\left(t_{0}, h\right)\right],
$$

where $\tilde{r}\left(t_{0}\right)=r\left(z\left(t_{0}\right)\right)$, etc., and $\tilde{\tau}[\tilde{m}]\left(t_{0}, h\right)=t_{0}+\frac{1}{2} h^{2} t_{0}^{-1} \tilde{m}\left(t_{0}\right)$. Note that we neglect the Jacobian of the coordinate change $2 / \tilde{c}\left(t_{0}\right)$. 
We also introduce the inverse of the traveltime function $\tilde{\tau}_{0}(t, h)$ such that $\tilde{\tau}\left(\tilde{\tau}_{0}(t, h), h\right)=$ $t$ and $\tilde{\tau}_{0}\left(\tilde{\tau}\left(t_{0}, h\right), h\right)=t_{0}$. For the inverse to exist globally, we need the traveltime to be strictly increasing as a function of $t_{0}$ :

$$
\tilde{s}\left(t_{0}, h\right)=\frac{\partial \tilde{\tau}}{\partial t_{0}}\left(t_{0}, h\right)>0 .
$$

The factor $\tilde{s}$ also determines the amount of coordinate stretch. Since this stretch is unphysical, we will want to restrict the range of $h$ such that $\tilde{s} \approx 1$. We have

$$
\tilde{s}\left(t_{0}, h\right)=1-\frac{h^{2}}{2 t_{0}^{2}}\left(t_{0} \frac{\mathrm{d} \tilde{m}}{\mathrm{~d} t_{0}}\left(t_{0}\right)-\tilde{m}\left(t_{0}\right)\right) .
$$

Typically, we use offsets $h \lesssim 10^{3} t_{0}$. In the remainder of the paper, we implicitly assume that an appropriate cut-off is applied whenever necessary, and neglect the factor $\tilde{s}$.

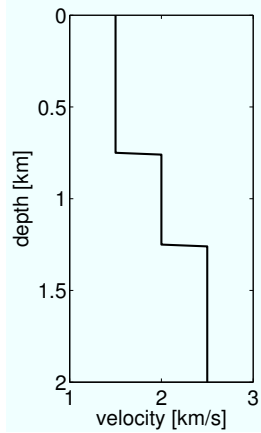

$[\mathrm{H}]$

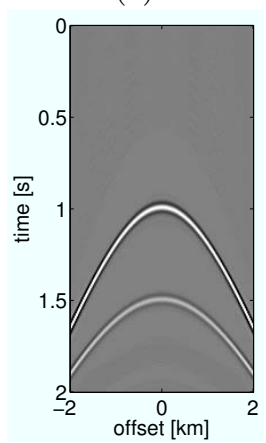

(d)

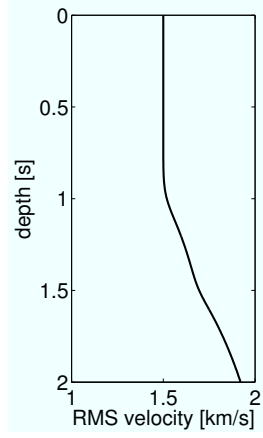

(b)

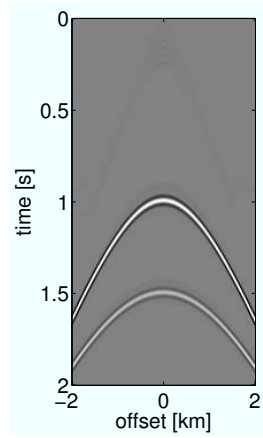

(e)

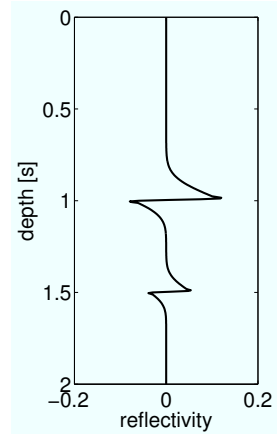

(c)

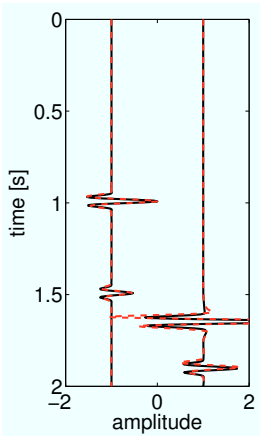

(f)

Figure 4.1. Example of data modelled with the convolutional model. (a) Velocity model c(z), (b) RMS velocity and (c) reflectivity $\tilde{r}\left(t_{0}\right)$. (d) Born data for velocity model (a) modelled with finite differences, (e) data for the convolutional model and (f) traces at $h=0 \mathrm{~km}$ (left) and $h=2 \mathrm{~km}$ (right) of the finite-difference and convolutional-model data (dash and solid lines respectively). 


\subsection{Linearized inversion}

Given the correct background velocity, the reflectivity can be retrieved from the data by solving the normal equations:

$$
N r=F^{*} p,
$$

where $N=F^{*} F$. The adjoint of the modelling operator can be directly derived from eq. (4.15), but it is insightful to derive it directly from eq. (4.11), using the adjoint-state technique. This yields:

$$
F^{*}\left[c_{0}\right] p=-\int \mathrm{d} \mathbf{x}_{r} \int \mathrm{d} \mathbf{x}_{s} \int \mathrm{d} \omega \omega^{2} u_{0}^{*}\left(\omega, \mathbf{x}_{s}, \mathbf{x}_{r}\right) v_{0}\left(\omega, \mathbf{x}_{s}, \mathbf{x}_{r}\right),
$$

where the source wavefield, $u_{0}$, satisfies eq. 4.4 and the receiver wavefield, $v_{0}$, satisfies

$$
L^{*}\left[c_{0}\right] v_{0}=\int \mathrm{d} \mathbf{x} p\left(\omega, \mathbf{x}_{r}, \mathbf{x}_{s}\right) \delta\left(\mathbf{x}-\mathbf{x}_{r}\right) .
$$

Under the assumptions mentioned above, the source and receiver wavefields are given by

$$
\begin{aligned}
& \tilde{u}_{0}[\tilde{m}]\left(\omega, t_{0}, h\right) \sim f(\omega) \exp \left[\imath \omega \frac{1}{2} \tilde{\tau}[\tilde{m}]\left(t_{0}, h\right)\right], \\
& \tilde{v}_{0}[\tilde{m}]\left(\omega, t_{0}, h\right) \sim p(\omega, h) \exp \left[-\imath \omega \frac{1}{2} \tilde{\tau}[\tilde{m}]\left(t_{0}, h\right)\right] .
\end{aligned}
$$

We arrive at

$$
\tilde{F}^{*} p=-\int \mathrm{d} h \int \mathrm{d} \omega \omega f^{*}(\omega) p(\omega, h) \exp \left[-\imath \omega \tilde{\tau}[\tilde{m}]\left(t_{0}, h\right)\right] .
$$

This operation is called 'NMO-correction', since it removes the 'moveout' effect in the data.

We study the normal operator, given by

$$
\begin{aligned}
\tilde{N}[\tilde{m}] \tilde{r} & =\int \mathrm{d} \omega \int \mathrm{d} t_{0}^{\prime} \int \mathrm{d} h \omega^{2}|f(\omega)|^{2} \tilde{r}\left(t_{0}^{\prime}\right) \exp \left[\imath \omega\left(\tilde{\tau}[\tilde{m}]\left(t_{0}^{\prime}, h\right)-\tilde{\tau}[\tilde{m}]\left(t_{0}, h\right)\right]\right. \\
& \sim \int \mathrm{d} t_{0}^{\prime} \int \mathrm{d} \omega \omega^{2}|f(\omega)|^{2} \tilde{r}\left(t_{0}^{\prime}\right) \exp \left[\imath \omega\left(t_{0}^{\prime}-t_{0}\right)\right] .
\end{aligned}
$$

In case the wavelet is a delta function, we have $\tilde{N} \tilde{r} \sim \frac{\mathrm{d}^{2}}{\mathrm{~d} t_{0}^{2}} \tilde{r}$. This result is consistent with a more general analysis of the normal operator Beylkin (1985), which states that the normal operator is asymptotically diagonal in phase space. Throughout the rest of the paper we assume that we have applied appropriate filtering to account for the $\omega^{2}$ factor.

\section{Example}

Fig. 4.1 shows a simple velocity model together with the corresponding effective velocity and reflectivity. Data modelled with a time-domain finite-difference code 
Mulder \& Plessix (2002) are depicted, as are the data resulting from the convolutional model operator (cf. eq. (4.15)). For comparison, two traces at $h=0 \mathrm{~m}$ and $h=2000 \mathrm{~m}$ are depicted, showing that the traveltimes of the events in the convolutional model data are accurate. The amplitudes are not correctly modelled, especially for large offsets.

\subsection{Velocity analysis in the image domain}

How do we get a handle on the velocity error? The linearized inverse problem mentioned above can be solved for any given background velocity, but the result will only be useful if the background velocity is correct. First, we observe that the source and receiver wavefields that constitute the reflectivity estimate will constructively interfere at the location of a reflector. Thus, at a reflector position, $t_{0}$, we have

$$
u_{0}\left(\omega, t_{0}, h\right) \sim v_{0}\left(\omega, t_{0}, h\right)
$$

(cf. eq. $(4.21,4.22)$ ). If the background velocity is incorrect, however, the wavefields will not match at a reflector. In image-domain velocity analysis this is exploited in various ways. We will now discuss several of them.

We will denote the observed data, and the corresponding 'true' reflectivity and background velocity with a bar, $\bar{p}=F[\bar{m}] \bar{r}$. Also, $\bar{\tau}=\tau[\bar{m}]$ and $\tau=\tau[m]$. The functional argument will be left out whenever there is no confusion possible.

\subsubsection{Stackpower}

The stackpower criterion relies on maximizing the inner product of the wavefields, which will attain a maximum if the wavefields are identical. The corresponding functional is formulated as Toldi (1989)

$$
J=\|r\|^{2}, \quad r\left(t_{0}\right)=\int \mathrm{d} h \int \mathrm{d} \omega u_{0}^{*}\left(\omega, t_{0}, h\right) v_{0}\left(\omega, t_{0}, h\right) .
$$

Using the expressions derived above we can write

$$
\begin{aligned}
& r\left(t_{0}\right)= \\
& \int \mathrm{d} h \int \mathrm{d} \omega \int \mathrm{d} t_{0}^{\prime} \bar{r}\left(t_{0}^{\prime}\right)|f(\omega)|^{2} \exp \left[\imath \omega\left(\left(t_{0}^{\prime}-t_{0}\right)+\frac{1}{2} h^{2}\left(\frac{\bar{m}\left(t_{0}^{\prime}\right)}{t_{0}^{\prime}}-\frac{m\left(t_{0}\right)}{t_{0}}\right)\right)\right] .
\end{aligned}
$$

The phase has a stationary point at $h=0$, implying that singularities in $\bar{r}$ are mapped onto themselves, or $r\left(t_{0}\right) \sim \bar{r}\left(t_{0}\right)$. The velocity error is to be found in the corresponding amplitude factor.

As an example, we present a scan of the functional. We use the data from the example presented before and evaluate the functional for different background velocities, which we represent on a 2 -node cubic spline grid $\left\{t_{0}, t_{1}\right\}=\{1,1.5\}$. Thus, we parameterize the background velocity with two parameters: $\left\{m_{1}, m_{2}\right\}$. We then evaluate the functional at $\left\{\bar{m}_{1}+\delta m_{1}, \bar{m}_{2}+\delta m_{2}\right\}$. As a source function we assume a 
point source with isotropic radiation pattern and a Ricker wavelet for the signature. In the frequency domain, the wavelet is given by Kanasewich (1985):

$$
f_{\epsilon}(\omega)=\omega^{2} \exp \left[-\epsilon \omega^{2}\right]
$$

The peak-frequency of this wavelet is $1 /(2 \pi \sqrt{\epsilon})$. This wavelet is commonly used in the geophysical community.

Figure 4.2 displays the stackpower for two different peak-frequencies, $5 \mathrm{~Hz}$ and 30 $\mathrm{Hz}$, as a function of the slowness perturbations. The stackpower is very sensitive to the frequency content of the data. As the frequency increases, the basin of attraction becomes smaller and local maxima start to develop. As seismic data are in general dominated by high frequencies, this approach is less suitable for velocity inversion.
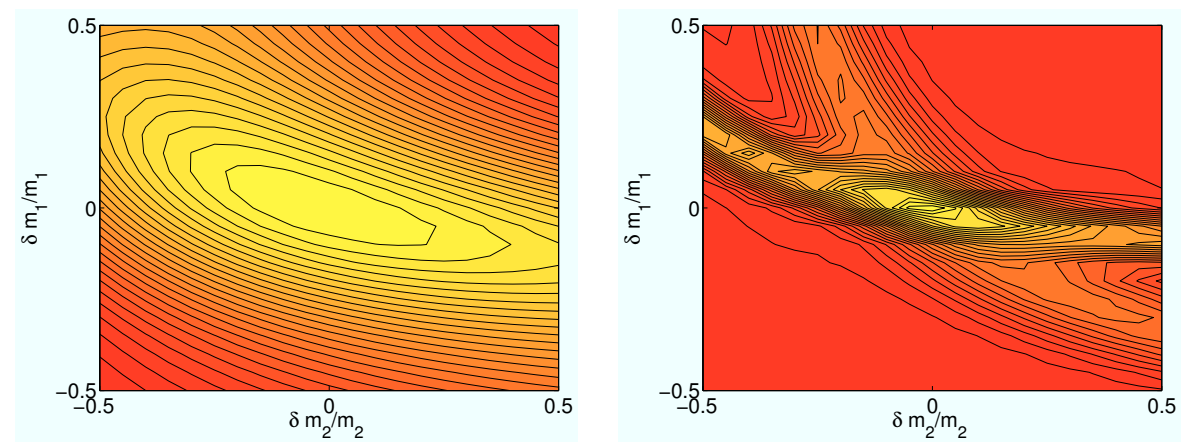

Figure 4.2. Stackpower functional (eq. (4.26)) as a function of the slowness perturbation $\left\{\delta m_{1}, \delta m_{2}\right\}$, for peak frequencies $5 \mathrm{~Hz}$ and $30 \mathrm{~Hz}$, respectively. As the peak frequency of the source increases, the behavior of the functional becomes more singular and local minima start to develop.

\subsubsection{Differential semblance}

An alternative way to measure the difference between the wavefields is the so-called differential semblance criterion. The idea is that we can obtain a reflectivity estimate from just a single trace and we can use the offset as the redundant coordinate. The reflectivity estimate effectively becomes a function of both depth and offset, denoted as $r\left(t_{0}, h\right)$. Note that the conventional reflectivity estimate is obtained by integrating $r\left(t_{0}, h\right)$ over offset. For the correct velocity model, the reflectivity estimate should be independent of offset. The velocity error can now be measured by differentiation w.r.t. offset, hence the name differential semblance. Here, the offsetdifferentiation is called an annihilator, since it annihilates the reflectivity estimate for the correct velocity model, i.e., $\partial_{h} r[\bar{m}]=0$. The corresponding error functional is given by

$$
J=\left\|\partial_{h} r\right\|^{2} .
$$

This approach was introduced by Symes (1991) and has been elaborated upon by others Chauris \& Noble (2001); Mulder \& ten Kroode (2002); Li \& Symes (2007). 
The reflectivity estimate is given by

$$
\begin{aligned}
& r\left(t_{0}, h\right)=\int \mathrm{d} \omega u_{0}^{*}\left(\omega, t_{0}, h\right) v_{0}\left(\omega, t_{0}, h\right) \\
& =\int \mathrm{d} \omega \int \mathrm{d} t_{0}^{\prime} \bar{r}\left(t_{0}^{\prime}\right)|f(\omega)|^{2} \exp \left[\imath \omega\left(\left(t_{0}^{\prime}-t_{0}\right)+\frac{1}{2} h^{2}\left(\frac{\bar{m}\left(t_{0}^{\prime}\right)}{t_{0}^{\prime}}-\frac{m\left(t_{0}\right)}{t_{0}}\right)\right)\right] \\
& \sim \bar{r}\left(t_{0}+\frac{h^{2}}{2 t_{0}}(\bar{m}-m)\right) .
\end{aligned}
$$

This shows that the reflectivity estimate is independent of $h$ for the correct velocity. Fig. 4.3 shows an example of $r\left(t_{0}, h\right)$ for different velocities.

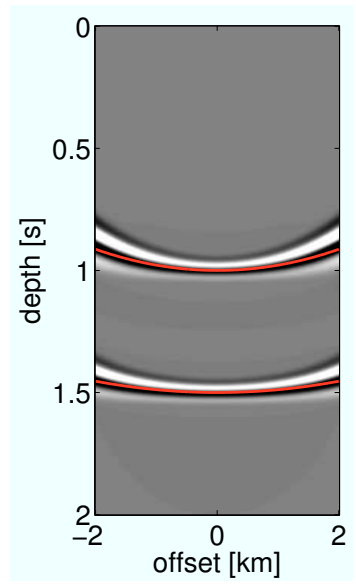

(a)

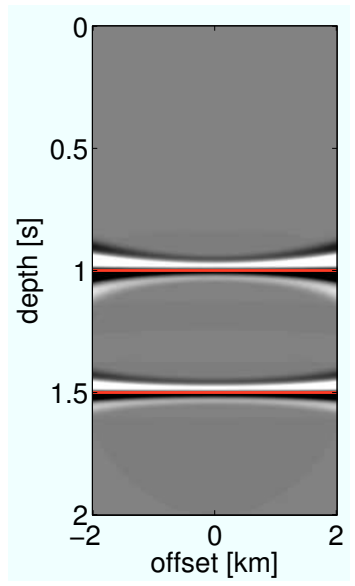

(b)

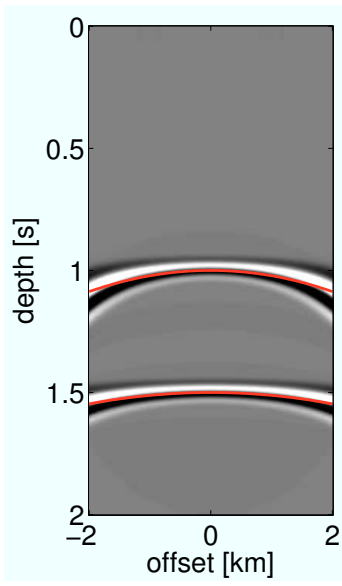

(c)

Figure 4.3. Reflectivity estimate $r\left(t_{0}, h\right)$, used for differential semblance, for a velocity that is $10 \%$ too low (a), correct (b) and $10 \%$ too high (c). The trajectories along which the singularities in the reflectivity are smeared out, cf. eq. (4.30), are indicated in red.

Fig. 4.4 depicts the behavior of the DSO functional as a function of the velocity error for different peak frequencies. It shows that the DSO functional has a large basin of attraction and does not depend on the frequency content of the data. In fact, Symes (1999) proved that, under similar assumptions as introduced here, the DSO functional is asymptotically stable. Numerical evidence exists that the same holds under more general conditions Chauris \& Noble (2001); Mulder \& ten Kroode (2002); Li \& Symes (2007).

Finally, an interesting observation can be made by considering the spatial Fourier transform of $r\left(t_{0}, h\right)$, denoted by $\hat{r}\left(t_{0}, k\right)$. Whereas the events will flatten in $r$, they will focus at $k=0$ in $\hat{r}$. It is readily verified that $\int \mathrm{d} h r\left(t_{0}, h\right)=\hat{r}\left(t_{0}, 0\right)$. This indicates that, in the stackpower approach, we are actually throwing away valuable information by integrating over offset. 

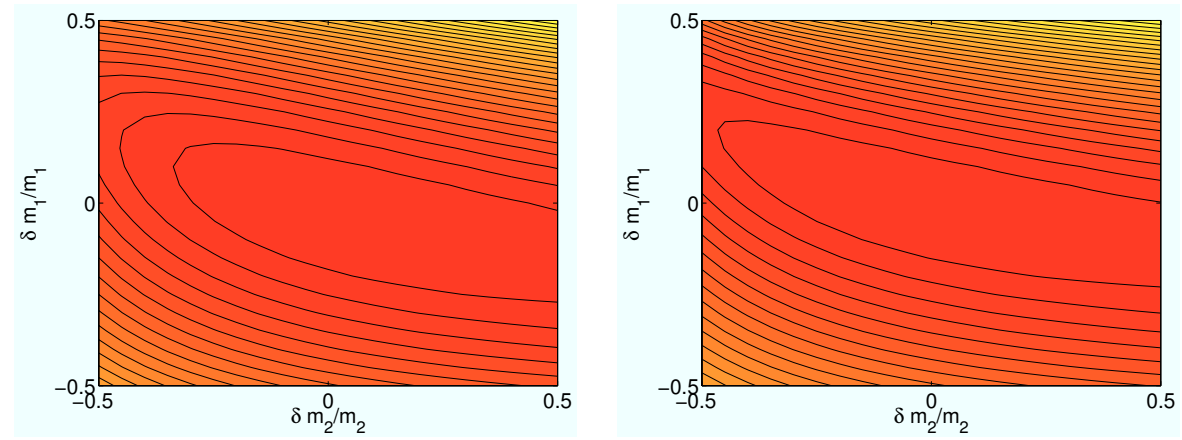

Figure 4.4. Differential semblance functional (eq. (4.29)) as a function of the slowness perturbation $\left\{\delta m_{1}, \delta m_{2}\right\}$, for peak frequencies $5 \mathrm{~Hz}$ and $30 \mathrm{~Hz}$, respectively. This functional does not depend on the frequency content of the data.

\subsubsection{Correlation}

A third way to measure the difference between the wavefields comes from the observation that a correlation of two identical signals will have a large contribution at zero lag. If there is a pertinent shift between the signals, the correlation will reveal this by having a large contribution at some non-zero lag. Introducing a temporal and spatial shift, we write the reflectivity estimate as

$$
r\left(t_{0}, \Delta t, \Delta h\right)=\int \mathrm{d} h \int \mathrm{d} \omega u_{0}^{*}\left(\omega, t_{0}, h\right) v_{0}\left(\omega, t_{0}, h+\Delta h\right) \exp [-\imath \omega \Delta t] .
$$

We can introduce the scattering angle as redundant coordinate by taking the angletransform of $r\left(r_{0}, \Delta t, \Delta h\right)$. The angle transform is defined as Stolk \& de Hoop (2006):

$$
r\left(t_{0}, \theta\right)=\int \mathrm{d} \Delta h r\left(t_{0}, \theta \Delta h, \Delta h\right) .
$$

For small $\Delta h$ this can be re-written as

$$
r\left(t_{0}, \theta\right) \sim \bar{r}\left(t_{0}+\frac{\theta^{2}}{2 m^{2} t_{0}}(\bar{m}-m)\right) .
$$

This may be seen as a generalization of the differential semblance method, discussed before. de Hoop et al. (2006) have proven rigorously that this approach is valid for laterally varying media as well.

Next we discuss the use of $\Delta h$ and $\Delta t$ separately as redundant coordinate.

\section{Spatial shift}

The use of the spatial shift for velocity analysis has been studied by many authors MacKay \& Abma (1992); Rickett \& Sava (2002); Sava \& Fomel (2006); Shen \& Symes (2008). Intuitively, the focusing may be understood by observing that the shift that is introduced actually represents an instantaneous displacement of the 
wavefield, or 'action at a distance'. This is unphysical and the correct velocity model does not allow for this, making the wavefields meet each other at zero shift. The amount of focus in the reflectivity can be now be measured by a multiplication by $\Delta h$, which serves as the annihilator. The misfit functional is defined accordingly as (Shen \& Symes, 2008):

$$
J=\|W \cdot r\|^{2}, \quad W(\Delta h)=\Delta h .
$$

The reflectivity estimate is given by

$$
\begin{aligned}
& r\left(t_{0}, \Delta h\right)= \\
& \int \mathrm{d} h \int \mathrm{d} \omega \int \mathrm{d} t_{0}^{\prime} \bar{r}\left(t_{0}^{\prime}\right)|f(\omega)|^{2} \exp \left[\imath \omega\left(\bar{\tau}\left(t_{0}^{\prime}, h\right)-\tau\left(t_{0}, h+\Delta h\right)\right)\right] .
\end{aligned}
$$

We examine the contribution to $r$ of a singularity in $\bar{r}$ by stationary phase analysis. A stationary point $h_{0}$ satisfies

$$
\frac{\partial \bar{\tau}}{\partial h}\left(t_{0}^{\prime}, h_{0}\right)-\frac{\partial \tau}{\partial h}\left(t_{0}, h_{0}+\Delta h\right)=0
$$

which yields

$$
h_{0}=\left(\frac{\bar{m}\left(t_{0}^{\prime}\right) t_{0}}{m\left(t_{0}\right) t_{0}^{\prime}}-1\right)^{-1} \Delta h
$$

The phase at the stationary point is given by

$$
\bar{\tau}\left(t_{0}^{\prime}, h_{0}\right)-\tau\left(t_{0}, h_{0}+\Delta h\right)=\left(t_{0}^{\prime}-t_{0}\right)-\frac{\Delta h^{2}}{2}\left(\frac{\bar{m}\left(t_{0}^{\prime}\right) m\left(t_{0}\right)}{\bar{m}\left(t_{0}^{\prime}\right) t_{0}-m\left(t_{0}\right) t_{0}^{\prime}}\right) .
$$

Note that the singularity that occurs at $m=\bar{m}$ is not a problem, since $\Delta h \rightarrow 0$ as $m \rightarrow \bar{m}$ as can be seen from eq. (4.37). With this we can express the reflectivity estimate as

$$
r\left(t_{0}, \Delta h\right) \sim \bar{r}\left(t_{0}+\frac{\Delta h^{2}}{2 t_{0}}\left(\frac{\bar{m} m}{\bar{m}-m}\right)\right) .
$$

This shows how a singularity in $\bar{r}$ is spread out along a certain trajectory in the $\left(t_{0}, \Delta h\right)$ space. For the correct velocity model, this trajectory collapses to a point at $\Delta h=0$ (cf. eq. (4.37)) and then $r\left(t_{0}, \Delta h\right) \sim \bar{r}\left(t_{0}\right) \delta(\Delta h)$. Fig. 4.5 displays an example. Fig. 4.6 shows the behavior of the functional as function of velocity error for different peak frequencies. Like the DSO functional, this functional has a large basin of attraction and is insensitive to the frequency content of the data.

Note that, analogously to the earlier observation, the conventional reflectivity estimate is obtained by evaluating $r\left(t_{0}, \Delta h\right)$ at $\Delta h=0$. 


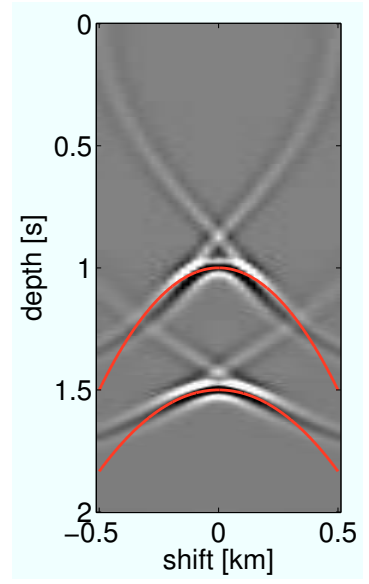

(a)

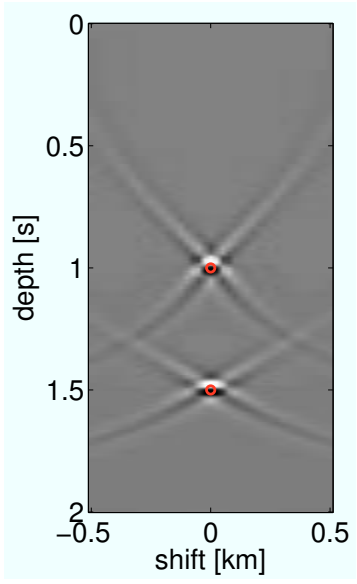

(b)

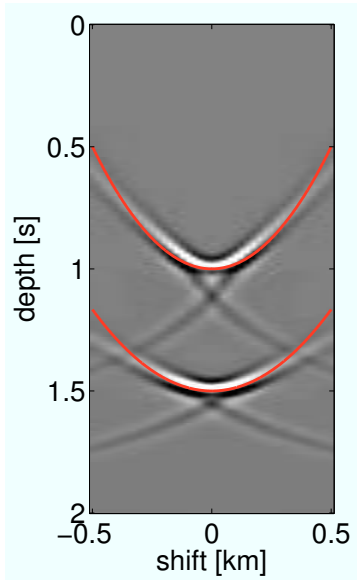

(c)

Figure 4.5. Reflectivity estimate $r\left(t_{0}, \Delta h\right)$, used in the spatial correlation, for a velocity that is $10 \%$ too low (a), correct (b) and $10 \%$ too high (c). The trajectories along which the singularities in the reflectivity are smeared out, cf. eq. (4.39), are indicated in red.
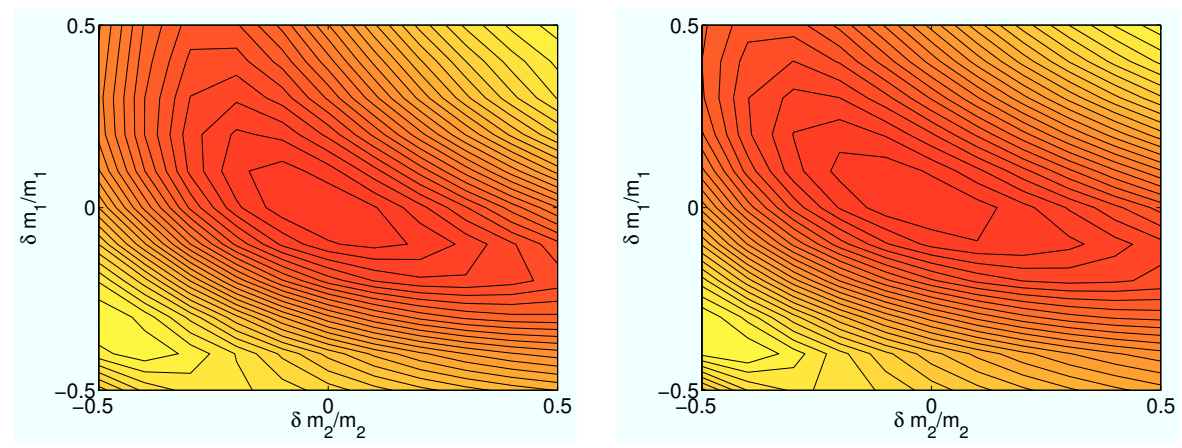

Figure 4.6. Spatial shift functional (eq. (4.34)) as a function of the slowness perturbation $\left\{\delta m_{1}, \delta m_{2}\right\}$, for peak frequencies $5 \mathrm{~Hz}$ and $30 \mathrm{~Hz}$, respectively. This functional does not depend on the frequency content of the data.

\section{Temporal shift}

The correlation with a temporal shift also exhibits a focusing behavior, though it is quite different from the spatial shift discussed previously. The reflectivity estimate is given by

$$
\begin{aligned}
& r\left(t_{0}, \Delta t\right)= \\
& \int \mathrm{d} h \int \mathrm{d} \omega \int \mathrm{d} t_{0}^{\prime} \bar{r}\left(t_{0}^{\prime}\right)|f(\omega)|^{2} \exp \left[\imath \omega\left(\bar{\tau}\left(t_{0}^{\prime}, h\right)-\tau\left(t_{0}, h\right)+\Delta t\right)\right] .
\end{aligned}
$$


Again, we calculate the stationary point $h_{0}$ from

$$
\frac{\partial \bar{\tau}}{\partial h}\left(t_{0}^{\prime}, h_{0}\right)-\frac{\partial \tau}{\partial h}\left(t_{0}, h_{0}\right)=0
$$

yielding $h_{0}=0$. The phase of the stationary point is now given by

$$
\bar{\tau}\left(t_{0}^{\prime}, 0\right)-\tau\left(t_{0}, 0\right)+\Delta t=t_{0}-t_{0}^{\prime}+\Delta t .
$$

So, singularities in $\bar{r}$ are spread out along straight lines, independent of the velocity error. The velocity dependence is to be found in the corresponding amplitude factor which depends on the second derivative of the phase function:

$$
\frac{\partial^{2} \bar{\tau}}{\partial h^{2}}\left(t_{0}^{\prime}, 0\right)-\frac{\partial^{2} \tau}{\partial h^{2}}\left(t_{0}, 0\right)=\frac{\bar{m}\left(t_{0}^{\prime}\right)}{t_{0}^{\prime}}-\frac{m\left(t_{0}\right)}{t_{0}} .
$$

With this, we may express the reflectivity estimate as

$$
r\left(t_{0}, \Delta t\right) \sim \sqrt{\left|\frac{\left(t_{0}+\Delta t\right) t_{0}}{t_{0} \bar{m}\left(t_{0}+\Delta t\right)-\left(t_{0}+\Delta t\right) m\left(t_{0}\right)}\right|} \bar{r}\left(t_{0}+\Delta t\right) .
$$

An example is shown in Fig. 4.7.

The location of the peak in amplitude at $\Delta t=(\bar{m} / m-1) t_{0}$ can be used to update the velocity model by determining the location of the peaks in the image automatically. A similar approach is described by Faye \& Jeannot (1986) and Higginbotham et al. (2008). Although in this case a multiplication by $\Delta t$ does not annihilate the reflectivity estimate, we define the functional analogously to eq. (4.34). The behavior of this functional is depicted in Fig. 4.8. This functional has the minimum in the wrong place. Although the amplitude peaks in the image are located at zero shift for the correct velocity model, the functional does not capture this. 


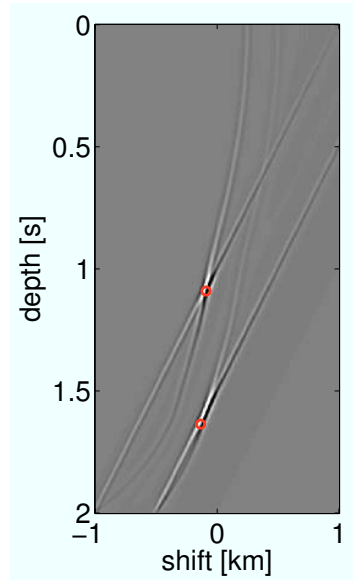

(a)

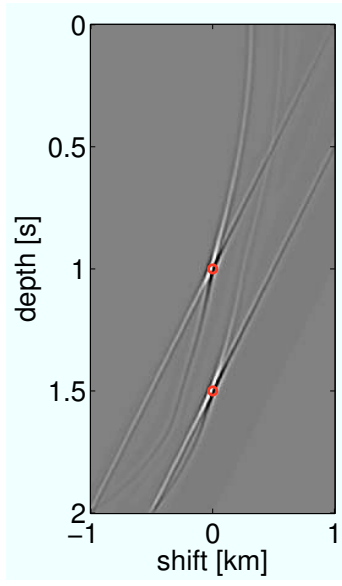

(b)

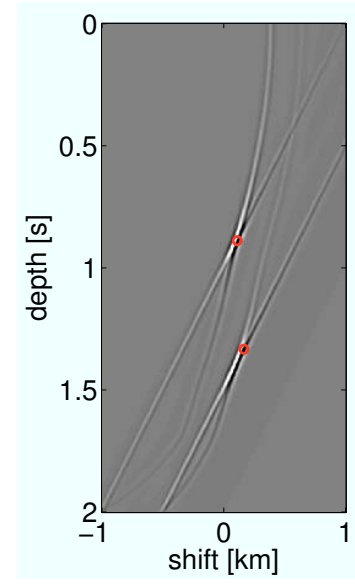

(c)

Figure 4.7. Reflectivity estimate $r\left(t_{0}, \Delta t\right)$, used for the temporal correlation, for a velocity that is $10 \%$ too low (a), correct (b) and $10 \%$ too high (c). The trajectories along which the singularities in the reflectivity are smeared out, cf. eq. (4.44), are indicated in red.
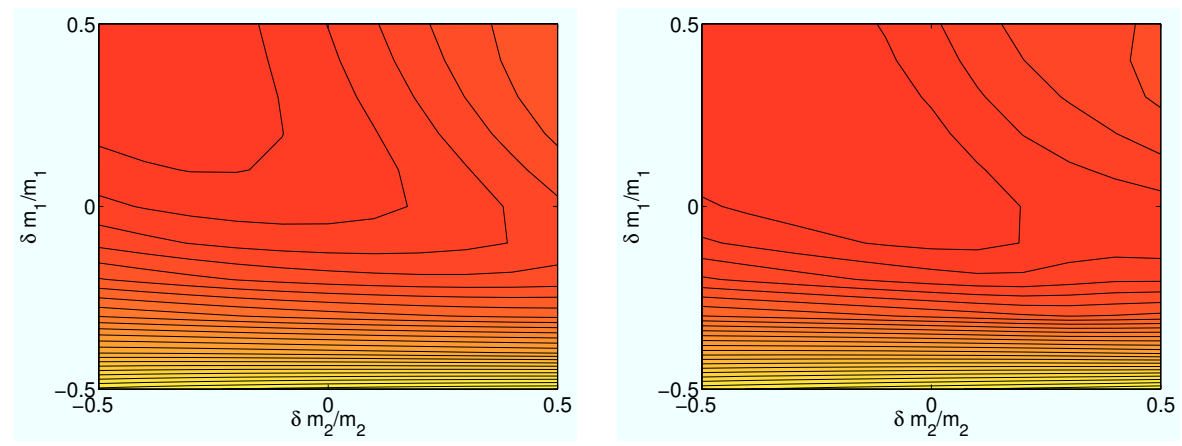

Figure 4.8. Temporal shift functional as a function of the slowness perturbation $\left\{\delta m_{1}, \delta m_{2}\right\}$, for peak frequencies $5 \mathrm{~Hz}$ and $30 \mathrm{~Hz}$, respectively. This functional does not appear to be suited for velocity analysis since the minimum is in the wrong place.

\subsection{Data domain velocity analysis}

A more intuitive approach to obtain the velocity model is to treat it as a non-linear least-squares problem. That is, we try to find $\{m, r\}$ that minimise

$$
J[m, r]=\|F[m] r-\bar{p}\|^{2} .
$$


This approach was pioneered by Tarantola \& Valette (1982) and elaborated on by many others. Exploiting the fact that the problem is separable, we can eliminate $r$ via the normal equations:

$$
r=N^{-1} F^{*} \bar{p}
$$

Substituting this into eq. (4.45) we obtain a modified functional:

$$
\tilde{J}[m]=\|(\pi[m]-I) \bar{p}\|^{2},
$$

where

$$
\pi[m]=F[m] N[m]^{-1} F^{*}[m],
$$

is an orthogonal projector onto the range of $F$. This approach is know as the variable projection approach Golub \& Pereyra (1973). Note that $(\pi-I)$ is an annihilator acting on the data, i.e., $(\pi[\bar{m}]-I) \bar{p}=0$.

We also note that the zero-offset trace of the data is invariant under the projection, that is

$$
\bar{p}(t, 0) \sim(\pi[m] \bar{p})(t, 0) .
$$

This follows immediately from the earlier observation that $F^{*} \bar{p} \sim \bar{r}\left(t_{0}\right)$.

As with the image-domain approach, we now have two wavefields, $\bar{p}$ and $\pi \bar{p}$, that will be identical for the correct velocity model. Next, we discuss several methods of comparing the two wavefields.
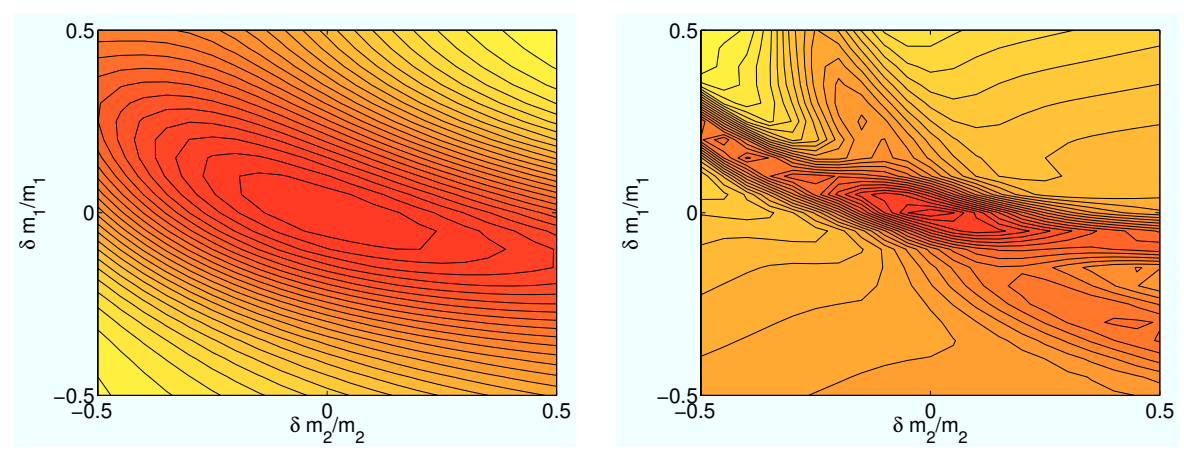

Figure 4.9. MBTT functional (eq. (4.47)) as a function of the slowness perturbation $\left\{\delta m_{1}, \delta m_{2}\right\}$, for peak frequencies $5 \mathrm{~Hz}$ and $30 \mathrm{~Hz}$, respectively. As the peak frequency increases, the behavior of the functional becomes less smooth.

\subsubsection{Least-squares}

The obvious way to match the wavefields is by minimising the modified least-squares functional. This approach is also known as Migration Based Traveltime Tomography (MBTT) and was first proposed by Clément et al. (2001). The functional may be written as

$$
\tilde{J}=\|\bar{p}\|^{2}-\langle\pi \bar{p}, \bar{p}\rangle=\|\bar{p}\|^{2}-\left\langle N^{-1} F^{*} \bar{p}, F^{*} \bar{p}\right\rangle \sim\|\bar{p}\|^{2}-\left\|F^{*} \bar{p}\right\|^{2} .
$$


This reveals that this approach is akin to the stackpower approach; the wavefields are matched by maximizing the inner product. Fig. 4.9 shows the behavior of the functional and confirms the assertion. This similarity also holds under more general conditions Stolk \& Symes (2003); Symes (2008b).

\subsubsection{Correlation}

Here, we use a correlation of the data and their projection to judge the similarity. As with the correlation of the wavefields $u_{0}$ and $v_{0}$ discussed before, the correlation of the data and their projection will have a large contribution at zero shift if the velocity is correct. In an earlier paper van Leeuwen \& Mulder (2008b), we successfully exploited this to obtain a velocity estimate. We consider a spatial and temporal shift.

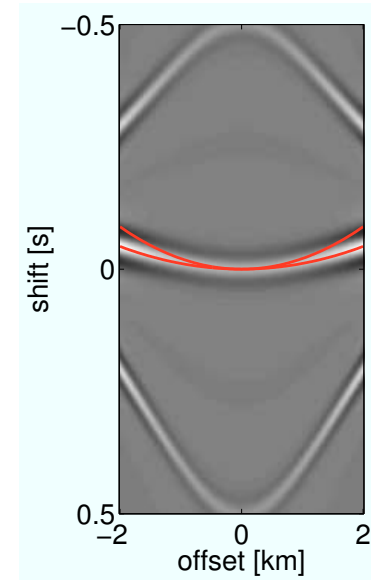

(a)

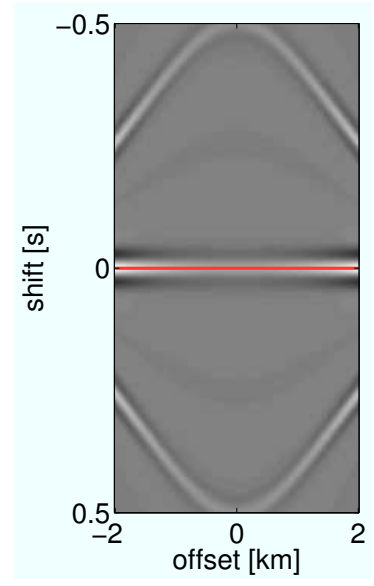

(b)

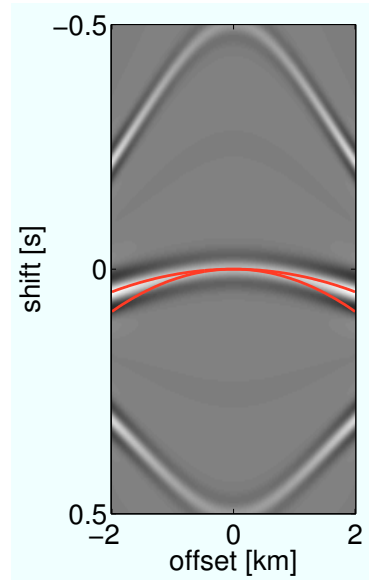

(c)

Figure 4.10. Data correlation $C_{h}$ for a velocity that is $10 \%$ too low (a), correct (b) and 10\% too high (c). The focusing events (cf. eq. (4.53)) are indicated in red while the non-focusing events are indicated in purple.

\section{Spatial shift}

Given data $\bar{p}$ and $p=\pi \bar{p}$, the spatial correlation is given by

$$
C_{h}(t, \Delta h)=\int \mathrm{d} h p(t, h) \bar{p}(t, h+\Delta h) .
$$

Since the zero-offset trace of the data is invariant under the projection we may write this as

$$
C_{h}(t, \Delta h) \sim \int \mathrm{d} h \bar{r}\left(\tau_{0}(t, h)\right) \bar{r}\left(\bar{\tau}_{0}(t, h+\Delta h)\right),
$$

We expect a significant contribution to $C_{h}$ if $\tau_{0}(t, h)=\bar{\tau}_{0}(t, h+\Delta h)$.

This relation allows us to investigate different events that turn up in the correlation. 
For the two-reflector example $\bar{r}\left(t_{0}\right)=\delta\left(t_{0}-T_{1}\right)+\delta\left(t_{0}-T_{2}\right)$, the contributions to $C_{h}$ are given by

$$
\Delta h= \pm\left(T_{i} \sqrt{\frac{t-T_{i}}{m\left(T_{i}\right)}} \pm T_{j} \sqrt{\frac{t-T_{j}}{\bar{m}\left(T_{j}\right)}}\right), \quad i, j \in\{1,2\}
$$

The contributions for $i=j$ can be interpreted as the result of the interaction of a reflector with itself. It shows that this interaction cause two distinct events in the correlation; one that will focus as $\bar{m} \rightarrow m$ and one that will not. Fig. 4.10 displays an example. The contributions for $i \neq j$ result from interaction between the different reflectors. This crosstalk is not present in Fig. 4.10 because it manifests itself at larger shifts. For a more detailed discussion of the crosstalk, we refer to section 8 and our earlier paper van Leeuwen \& Mulder (2008a).

Unfortunately, because of the non-focusing events, we cannot formulate an annihilator. Instead, we adopt a heuristic approach, measuring the amount of energy concentrated around zero shift with the functional:

$$
J[m]=\frac{\left\|W_{h} \cdot C_{h}\right\|^{2}}{\left\|C_{h}\right\|^{2}}, \quad W_{\sigma}(\Delta h)=\exp \left[-\sigma \Delta h^{2}\right]
$$

Here, $\sigma$ determines the width of the weighting function. Note that this functional is to be maximise. Fig. 4.11 depicts the behavior of this functional. The functional is smooth as a functional of the velocity error and has a large basin of attraction. Also, the behavior does not change significantly with the frequency.
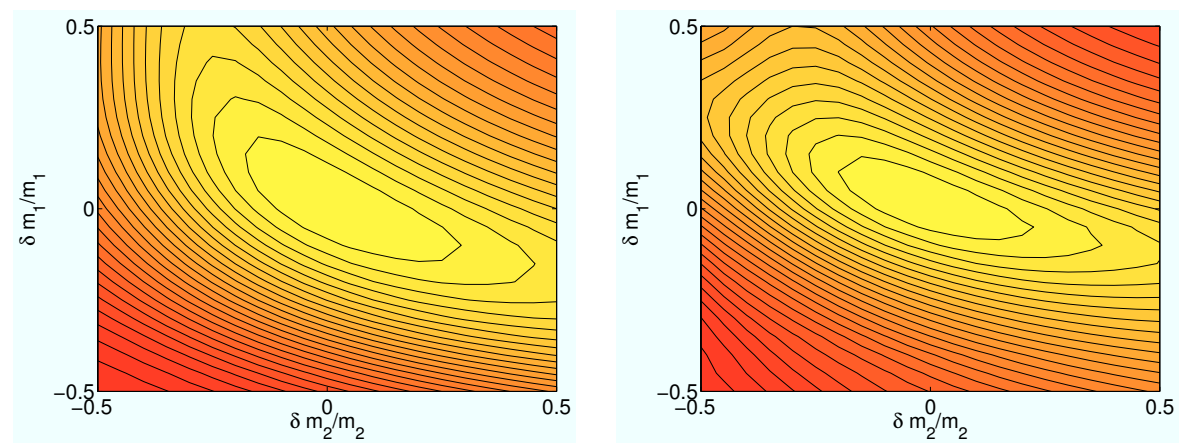

Figure 4.11. Spatial correlation functional (eq. (4.54)) as a function of the slowness perturbation $\left\{\delta m_{1}, \delta m_{2}\right\}$, for peak frequencies $5 \mathrm{~Hz}$ and $30 \mathrm{~Hz}$, respectively. The functional does not change its behavior dramatically as the frequency content of the data changes.

\section{Temporal shift}

We also consider the temporal correlation given by

$$
C_{t}(\Delta t, h)=\int \mathrm{d} t p(t, h) \bar{p}(t+\Delta t, h)
$$


which may be written as

$$
C_{t}(\Delta t, h)=\int \mathrm{d} t \bar{r}\left(\tau_{0}(t, h)\right) \bar{r}\left(\bar{\tau}_{0}(t+\Delta t, h)\right) .
$$

Again, we expect a contribution when $\tau_{0}(t, h)=\bar{\tau}_{0}(t+\Delta t, h)$. For our two-reflector model, $\bar{r}\left(t_{0}\right)=\delta\left(t_{0}-T_{1}\right)+\delta\left(t_{0}-T_{2}\right)$, this yields

$$
\Delta t=\left(T_{i}-T_{j}\right)+\frac{h^{2}}{2}\left(\frac{\bar{m}\left(T_{i}\right)}{T_{i}}-\frac{m\left(T_{j}\right)}{T_{j}}\right), \quad i, j \in\{1,2\} .
$$

So, for $i=j$ each reflector contributes one event to the correlation. This event will focus for the correct velocity model. As in the spatial correlation, there is crosstalk present. Fig. 4.12 depicts an example. Here, the crosstalk is also visible.

Again, we cannot formulate an annihilator for the temporal correlation. So, we adopt the heuristic approach, described above and define the functional analogous to eq. (4.54). Fig. 4.13 depicts its behavior. This functional has a large basin of attraction and its behavior does not change with frequency.

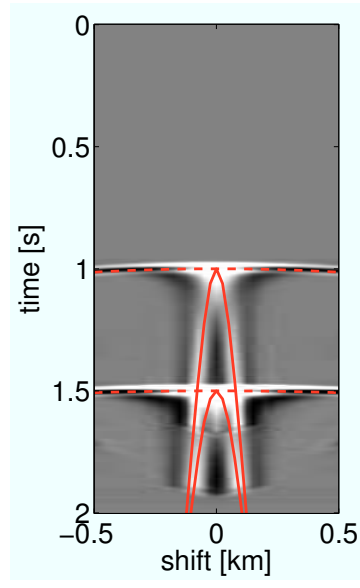

(a)

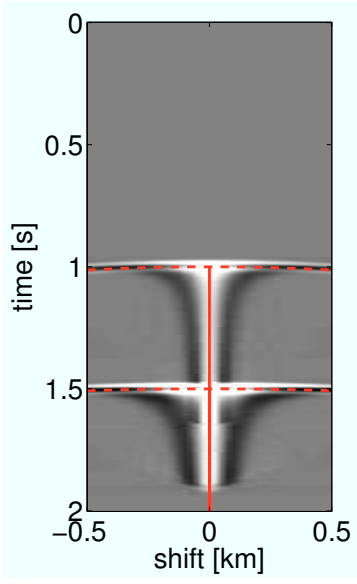

(b)

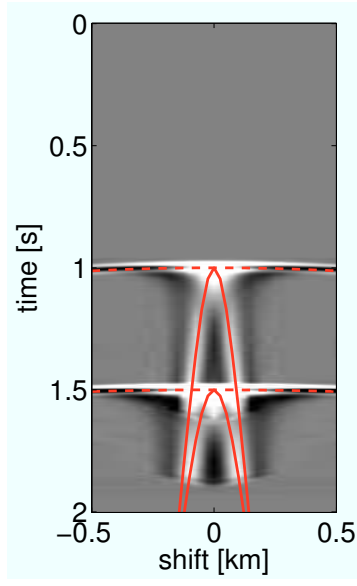

(c)

Figure 4.12. Data correlation $C_{t}$ for a velocity that is $10 \%$ too low (a), correct (b) and $10 \%$ too high (c). The focusing events, cf. eq. (4.44), are indicated in red. Note the presence of the crosstalk. 

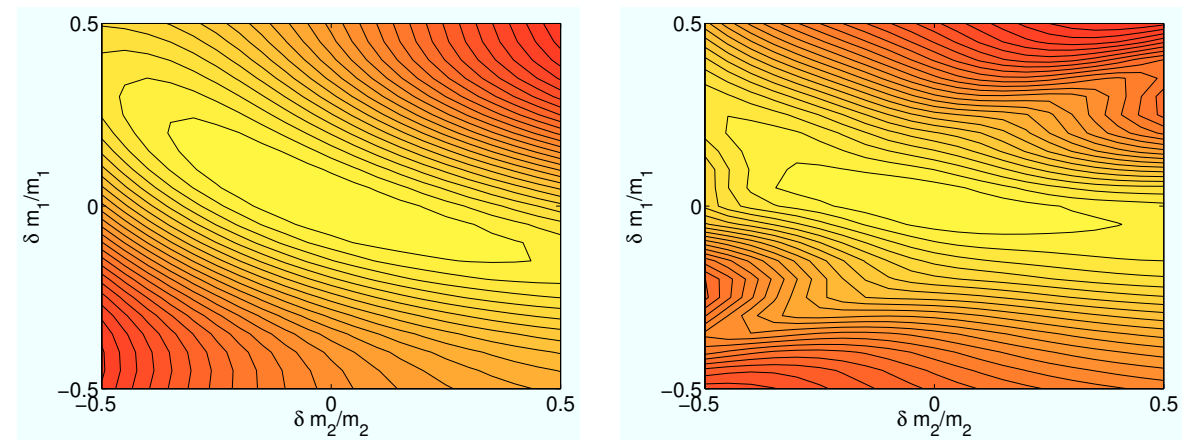

Figure 4.13. Temporal correlation functional (eq. (4.54)) as a function of the slowness perturbation $\left\{\delta m_{1}, \delta m_{2}\right\}$, for peak frequencies $5 \mathrm{~Hz}$ and $30 \mathrm{~Hz}$, respectively. The functional does not change its behavior dramatically as the frequency content of the data changes.

\subsection{Focusing in the image and data domain}

In this section, we will study the focusing of the spatial correlation in the image and data-domain more closely. We have seen that the focusing of the extended reflectivity $r\left(t_{0}, \Delta h\right)$ is described by stationary points of the phase function. There is a contribution to this image when the derivatives of the traveltimes are equal:

$$
\frac{\partial \bar{\tau}}{\partial h}\left(t_{0}, h\right)=\frac{\partial \tau}{\partial h}\left(t_{0}, h+\Delta h\right)
$$

which yields (cf. eq. (4.37))

$$
\Delta h=-\left(1+\sqrt{\frac{\bar{m}}{m}}\right)\left(1-\sqrt{\frac{\bar{m}}{m}}\right) h .
$$

There is a contribution to the correlation $C_{h}(t, \Delta h)$ when $\bar{\tau}_{0}(t, h)=\tau_{0}(t, h+\Delta h)$. For a fixed $t_{0}$ this is equivalent to

$$
\bar{\tau}\left(t_{0}, h\right)=\tau\left(t_{0}, h+\Delta h\right),
$$

which yields

$$
\Delta h=-\left(1 \pm \sqrt{\frac{\bar{m}}{m}}\right) h .
$$

Fig. 4.14 displays an example of the phase function $\bar{\tau}\left(t_{0}, h\right)-\tau\left(t_{0}, h+\Delta h\right)$ for a fixed $t_{0}$ and various relative velocity perturbations. This shows how the contributions to $r\left(t_{0}, \Delta h\right)$ and $C_{h}(t, \Delta h)$ gradually shift towards $\Delta h=0$ as the velocity error decreases. Effectively, by weighting with $\Delta h$ and taking the norm, the slope of the line is measured. In the DSO approach, the slope of the line is measured by taking 
the $h$-derivative at $\Delta h=0$. The non-focusing artifact that appears in the data correlation is also explained by these pictures; one of the solid lines hardly changes slope and is due to contributions of small offsets. This suggests that the artifact may be suppressed by removing the small offsets from the data.

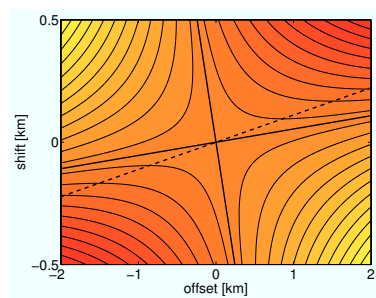

(a)

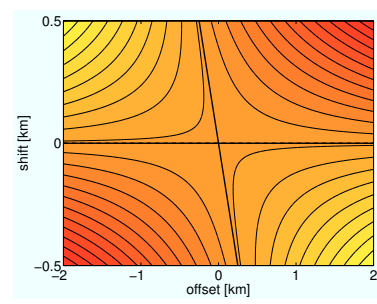

(b)

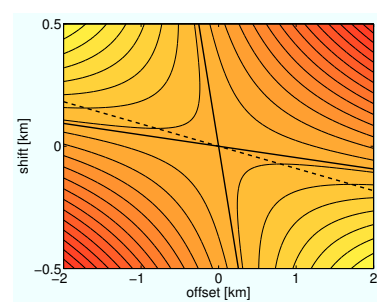

(c)

Figure 4.14. Phase function $\bar{\tau}\left(t_{0}, h\right)-\tau\left(t_{0}, h+\Delta h\right)$ at a fixed $t_{0}$ for a velocity that is (a) $10 \%$ too low, (b) correct and (c) $10 \%$ too high. The solid lines indicate where the phase function is zero. One of the trajectories moves towards $\Delta h=0$ as $m \rightarrow \bar{m}$ and creates the focusing event in the data correlation. The other trajectory creates the non-focusing artifact in the data correlation. This analysis suggests that this artifact is caused by the small offsets. The dotted line indicates where the derivative of the phase function is zero. The trajectory moves towards $\Delta h=0$ as $m \rightarrow \bar{m}$ and drives the focusing in the image-domain.

\subsection{Synthetic-data example}

We applied the differential semblance method and the spatial data-correlation, described above to synthetic data. The data where produced by solving the linearized wave equation for a 1D-slice of the Marmousi velocity model, a well-known benchmark model Versteeg (1993), with a time-domain finite-difference code. The RMS velocity was represented using cubic splines on an equidistant grid of 7 nodes. For the optimisation, we used a BFGS (Broyden-Fletcher-Shanno-Goldfarb) algorithm. The gradients of the functionals were calculated using the adjoint-state technique Plessix (2006).

The functional for the data-domain is slightly different from the one defined earlier:

$$
J=\left\|S^{-1} \cdot W_{\sigma} \cdot C_{h}\right\|^{2}, \quad S(t)=\int \mathrm{d} \Delta h\left(C_{h}(t, \Delta h)\right)^{2} .
$$

The scaling factor $S$ normalizes the amplitudes of the events.

Since the zero-offset trace of the data is invariant under the projection, we may write the projection of the data as

$$
\pi[m] \bar{p}=F[m]\left(\bar{p}\left(t_{0}, 0\right)\right) .
$$

Fig. 4.15 depicts the observed data as well as the true, initial, and final velocity model that resulted from optimizing the functional defined in eq. (4.62). The velocity model is almost perfectly recovered. A perfect fit is not to be expected, 
since we are representing the velocity model with splines. Fig. 4.17 shows the spatial correlation of the data and their projection for the initial and final velocity, illustrating how the focusing is improved.

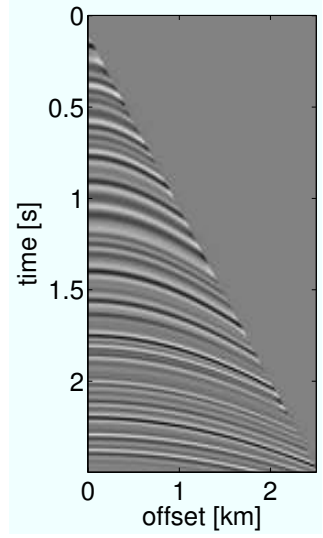

(a)

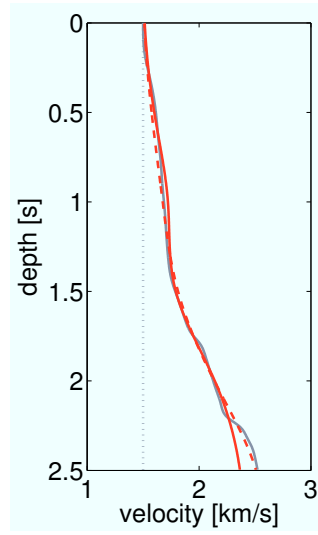

(b)

Figure 4.15. Synthetic-data example. The simulated data are generated with the convolutional model, using the first trace of the data as reflectivity and a Ricker wavelet with a peak frequency of $30 \mathrm{~Hz}$. (a) Shows the synthetic data and (b) shows the initial (dashed) and final (solid) velocity model that resulted from the $D S O$ (red) and data-correlation method(blue). The true RMS velocity is depicted in grey. The match is not prefect because we represent the NMO velocity with cubic splines. 


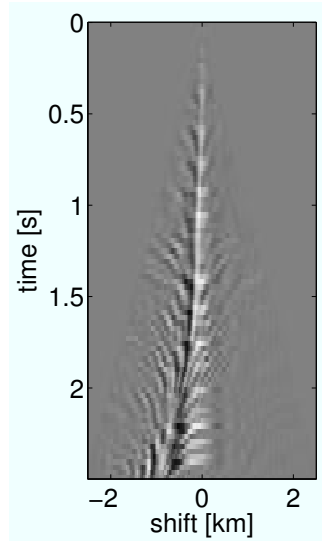

(a)

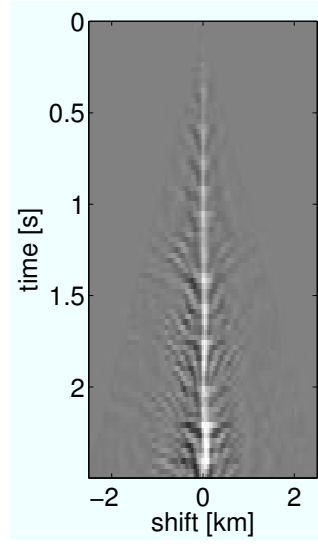

(b)

Figure 4.16. Data correlation for the synthetic-data example from Fig. 4.15 (a). The spatial correlation is depicted for the initial (left) and final (right) velocity model (4.15 (b), solid blue). The latter is clearly focused, whereas the former is not. It seems that the focusing is not quite as perfect around $t=2.25 \mathrm{~s}$. This probably due to the rapid variations in the RMS velocity model that could not be captured by splines.

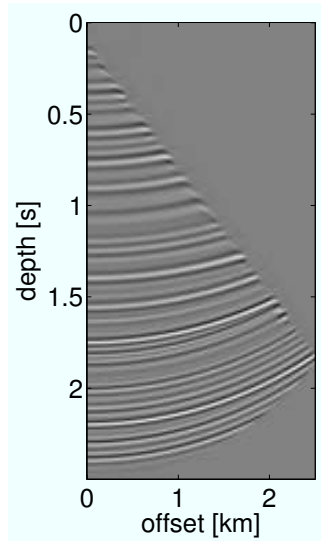

(a)

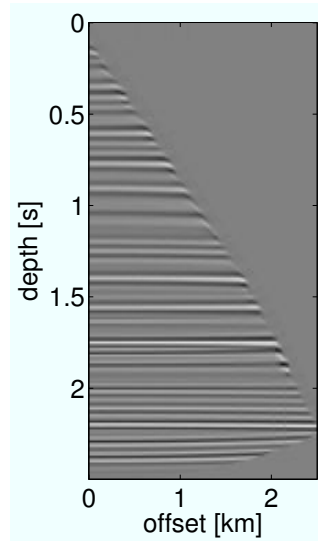

(b)

Figure 4.17. Reflectivity estimate $r\left(t_{0}, h\right)$ for the synthetic-data example from Fig. 4.15 (a). The reflectivity is depicted for the initial (left) and final (right) velocity model ( 4.15 (b), solid red). The latter is clearly 'flat', whereas the former is not. 


\subsection{Conclusions}

The seismic inverse problem, where we want to recover the soundspeed in the subsurface, can be cast as a separable least-squares problem. The resulting optimisation problem is linear in the oscillatory component of the velocity model and non-linear in the smooth background velocity. Typically, local minima exist and this requires a reformulation of the non-linear optimisation problem. We have reviewed several such alternative formulations, concentrating mainly on an intuitive understanding of the underlying principles of the different methods.

The methods discussed fall in two categories; methods that rely on amplitude fitting and methods that rely on phase fitting. The least-squares and stack-power approach, discussed in sections 5.1 and 6.1, are both relying on amplitude information to obtain the correct velocity model. These methods turn out to be very sensitive to the frequency content of the data, as can be seen in figs. 4.2 and 4.9. For higher peak frequencies the basin of attraction of the functionals becomes smaller and local minima start to develop. Also, one can imagine that field data will not be completely model-consistent. That is, even for the correct background velocity and reflectivity, the modelled amplitudes may not completely match the observed amplitudes. In this case, fitting the amplitudes may lead to a wrong background velocity. The other methods discussed rely on fitting the phases of the data. The phases, or traveltimes, of the data are expected to carry more information about the background velocity than the amplitudes do. Also, this approach is far less sensitive to the frequency content of the data. This is revealed in figs 4.4,4.6,4.11, and 4.13. The basin of attraction is large, independent of the peak frequency.

Additional analysis of two methods that use a spatial correlation reveals how the correlation will gradually focus as the velocity error decreases. Of course, one expects the traveltimes of the data to change gradually with velocity, and this translates to the focusing of the correlation, which basically measures a traveltime difference.

Although the analysis here is limited to layered media, the general principles of amplitude and phase fitting are still valid in laterally varying media. Therefore, we expect that the general conclusions will hold. In fact, for some of the methods abundant numerical evidence exists that is consistent with this analysis Chauris \& Noble (2001); Mulder \& ten Kroode (2002); Shen \& Symes (2008). Based on the analysis it is impossible to conclude which would be the preferred method, although it is clear that a reformulation in terms of phase fitting is beneficial for these kind of band-limited inverse problems. The computational complexity would also very much depend on the implementation and the modelling kernel used to solve the wave equation. 


\title{
A correlation-based misfit criterion for wave-equation traveltime tomography.
}

\author{
T.van Leeuwen and W.A. Mulder. 2010. Geophysical Journal International (in print).
}

\begin{abstract}
Wave-equation traveltime tomography tries to obtain a subsurface velocity model from seismic data, either passive or active, that explains their traveltimes. A key step is the extraction of traveltime differences, or relative phase shifts, between observed and modelled finite-frequency waveforms. A standard approach involves a correlation of the observed and measured waveforms. When the amplitude spectra of the waveforms are identical, the maximum of the correlation is indicative of the relative phase shift. When the amplitude spectra are not identical, however, this argument is no longer valid. We propose an alternative criterion to measure the relative phase shift. This misfit criterion is a weighted norm of the correlation and is less sensitive to differences in the amplitude spectra. For practical application it is important to use a sensitivity kernel that is consistent with the way the misfit is measured. We derive this sensitivity kernel and show how it differs from the standard banana-doughnut sensitivity kernel. We illustrate the approach on a cross-well data set.
\end{abstract}




\subsection{Introduction}

In ray-based tomography, the aim is to construct a subsurface velocity model that explains the picked traveltimes of the measured data. Such a model can be obtained in an iterative manner by back projecting the traveltime differences along rays in the current velocity model. This procedure will lead to satisfactory results when the wave propagation is sufficiently well approximated by ray theory. To extract more information from the data than just the traveltimes of a few selected arrivals, seismologists are moving towards full-waveform processing and inversion of all available data. This trend is driven by the availability of high-quality broadband data (earthquake data from USArray, for example) and a need to incorporate finite-frequency effects to process data from geologically complex areas (sub-salt exploration for the detection of hydrocarbons, for example). Also, the computing resources needed to routinely model $3-\mathrm{D}$ wave-propagation in complex media are becoming a commodity. There have been two major developments regarding finitefrequency or full-waveform inversion in the last decades. The first is least-squares inversion - or waveform tomography - of seismic data pioneered by Tarantola \& Valette (1982) which was aimed originally at inversion of active seismic reflection data. The second is the development of a finite-frequency analogue of ray-based traveltime tomography by Luo \& Schuster (1991) (see also Tromp et al., 2005). Both aim at exploiting the full measured waveforms by posing the inversion as an optimisation problem:

Given observed data, $\bar{d}$, find a subsurface model for which the modelled data, d, minimises the misfit functional $\phi[\bar{d}, d]$

The typical size of such optimisation problems dictates that we employ an iterative, gradient-based optimisation algorithm, such as non-linear Conjugate Gradients or a (Quasi-) Newton method (Vogel, 2002), to solve the optimisation problem. These methods require that the misfit does not exhibit local minima and has a large basin of attraction around the global minimum.

Whereas the least-squares misfit satisfies these criteria in some cases, resolving velocity structures that vary on a scale larger than the largest wavelength can be problematic. The basic rationale is as follows. These large scale velocity variations cause significant phase shifts between the modeled and observed waveforms. This, in turn, may cause local minima in the least-squares error through loop-skipping. Because the traveltimes are more linearly related to such velocity perturbations (Cara \& Leveque, 1987), one usually resorts to (wave-equation) traveltime tomography to resolve these structures. One way of measuring the misfit relies on picking the traveltime difference from the correlation of the modeled and measured waveforms (Luo \& Schuster, 1991; de Hoop \& van der Hilst, 2005).

In this paper, we propose a new correlation-based misfit functional that can be used for wave-equation traveltime tomography. We show that the proposed misfit functional implicitly measures the phase shift between the modelled and observed waveforms. Hence, the procedure is in principle equivalent to the correlation-based approach proposed by Luo \& Schuster (1991) where the phase shift is measured ex- 
plicitly by picking the maximum of the correlation. However, picking the maximum of the correlation will only reveal the phase shift correctly if the source spectra of the modelled and observed data are identical (Hörmann \& de Hoop, 2002; de Hoop \& van der Hilst, 2005). Our alternative procedure is less sensitive to errors in the estimated source spectrum.

As has been recognized by de Hoop \& van der Hilst (2005), such measures of misfit will only lead to meaningful updates of the model if one uses a sensitivity kernel that is consistent with the misfit. We derive the sensitivity kernel for the newly proposed measure of misfit. The exposition and analysis closely follows de Hoop \& van der Hilst (2005), and we repeat some of the main points and ideas of this work throughout the paper for completeness' sake.

The outline of the paper is as follows. First we briefly introduce some notation and describe the mathematical modelling used for the subsequent exposition and analysis. In section 3, we introduce the new correlation-based misfit functional, which is based on the weighted norm of the correlation, and compare it with a commonly used approach. We analyze them in some detail and show that the weighted norm of the correlation also measures the traveltime difference and is less sensitive to errors in the estimated source spectrum. The corresponding sensitivity kernels are derived and analyzed in section 4 . We calculate the kernels explicitly for a velocity that increases linearly with depth and use these to illustrate their properties in section 5. An inversion of real cross-well data using the proposed correlation-based approach is presented in section 6 . Finally, we discuss the results and present the conclusions.

\subsection{Modelling}

The displacement, $\mathbf{u}(t, \mathbf{x})$, is modelled by a linear elastic wave equation of the form

$$
\left[\delta_{i l} \partial_{t}^{2}-\partial_{x_{j}} c_{i j k l} \partial_{x_{k}}\right] u_{l}=f_{i}
$$

where $c_{i j k l}$ is the stiffness tensor and $f_{i}$ is the source function. The data consists of measurements of one or more components of the displacement for several distinct source-receiver pairs. Generally, waveform/traveltime tomography relies on solving the mentioned wave equation by some numerical method (e.g., finite differences). However, for the subsequent analysis we make a high-frequency assumption. We are interested in high-frequency body waves that, away from caustics, permit the following far-field representation of the Green's function

$$
G_{i}^{(j)}\left(t, \mathbf{x}_{s}, \mathbf{x}_{r}\right) \simeq(2 \pi)^{-1} a_{i}^{(j)}\left(\mathbf{x}_{s}, \mathbf{x}_{r}\right) \int \mathrm{d} \omega \exp \left[\imath \omega\left(t-T^{(j)}\left(\mathbf{x}_{s}, \mathbf{x}_{r}\right)\right)-\frac{n \pi}{2}\right],
$$

where $a$ is the amplitude, $T$ the traveltime, $n$ the KMAH index (indicating the number and type of caustics that the wave has encountered along the way) and $(j)$ enumerates the modes and traveltime branches. For the analysis we focus the exposition to one particular component, mode, and branch. We will assume point sources and denote the corresponding source spectrum by $f$, which we assume to be hermitian (i.e., $f(\omega)=f^{*}(-\omega)$ ) and square-integrable (i.e., has finite energy). Also, 
we ignore the factor $\exp [\imath n \pi / 2]$ for brevity of notation. Hence, we are considering data of the form

$$
d\left(t, \mathbf{x}_{s}, \mathbf{x}_{r}\right) \simeq(2 \pi)^{-1} a\left(\mathbf{x}_{s}, \mathbf{x}_{r}\right) \int \mathrm{d} \omega f(\omega) \exp \left[\imath \omega\left(t-T\left(\mathbf{x}_{s}, \mathbf{x}_{r}\right)\right]\right.
$$

We will drop the dependence on the source/receiver coordinates, denoting $T=$ $T\left(\mathbf{x}_{s}, \mathbf{x}_{r}\right)$, but $T_{s}=\left(\mathbf{x}_{s}, \mathbf{x}\right)$ etc. Also, we will not distinguish between perturbations of different components of the stiffness tensor, simply denoting the relevant (scalar) medium parameter by $c$. The measured or true quantities are denoted with a bar. Hence, $\bar{d}$ denotes the measured data, $\bar{c}$ denotes the true velocity model, etc.

Note that the above definitions imply the following conventions for the Fourier transform:

$$
\begin{aligned}
\hat{g}(\omega) & =\int \mathrm{d} t g(t) \exp [-\imath \omega t], \\
g(t) & =\frac{1}{2 \pi} \int \mathrm{d} \omega \hat{g}(\omega) \exp [\imath \omega t],
\end{aligned}
$$

which is the conjugate of what is usually used in geophysics.

\subsection{Correlation-based criteria}

An important ingredient of a wave-equation traveltime tomography scheme is the procedure to detect the relative phase shift between two waveforms. A straightforward approach relies on picking the relevant arrivals in the waveforms. More sophisticated techniques rely on correlating the waveforms before picking (Cara \& Leveque, 1987, for example). Since we are casting the tomography as an optimisation problem we seek to define a functional that attains a minimum - or maximum when the relative phase shift between the observed and modelled data is zero. Next, we discuss two such functionals that are based on the correlation of the measured and observed data. Part of this exposition can also be found in Luo \& Schuster (1991) and de Hoop \& van der Hilst (2005) and is repeated here for sake of completeness.

The correlation of the observed and modelled data is given by

$$
C[\bar{d}, d](t)=\int \mathrm{d} t^{\prime} \bar{d}\left(t^{\prime}+t\right) d\left(t^{\prime}\right)
$$

Assuming the data are of the form presented in eq (5.3) we have:

$$
\begin{aligned}
C[\bar{d}, d](t) & \simeq(2 \pi)^{-1} \bar{a} a \int \mathrm{d} \omega \hat{F}(\omega) \exp [\imath \omega(t-\Delta T)] \\
& =F(t-\Delta T)
\end{aligned}
$$

where $\Delta T=\bar{T}-T$ denotes the phase shift and $\hat{F}=\bar{f} f^{*}$ is the correlation of the source spectra. We will drop the amplitude factor $a \bar{a}$ in the rest of the paper. 


\subsubsection{Picking approach}

Luo \& Schuster (1991) proposed to extract the relative phase shift between the data by picking the maximum of the correlation:

$$
\Delta t=\underset{t}{\operatorname{argmax}} C[\bar{d}, d](t)
$$

The corresponding misfit functional is given by

$$
\phi_{\max }=(\Delta t)^{2}
$$

However, as has been noted by Hörmann \& de Hoop (2002) and de Hoop \& van der Hilst (2005), this approach is only strictly valid if the source spectra of the observed and modelled data are identical. Indeed, eq (5.7) suggests that the correlation has a maximum at $t=\Delta T$ when $F(t)$ has its maximum at $t=0$. If, for example, the source spectra differ by a phase rotation over $\pi / 2, F$ is an odd function with a zero crossing at $t=0$. Different scenarios are illustrated in figs 5.1-5.3(a). To indicate the difference between the picked and true traveltime difference explicitly, we introduce

$$
\epsilon_{T}=\Delta T-\Delta t
$$

Hence, the misfit functional, as a function of the phase shift $\Delta T$, behaves as

$$
\phi_{\max } \simeq\left(\Delta T+\epsilon_{T}\right)^{2} .
$$

Minimising this will lead to an erroneous velocity when $\epsilon_{T} \neq 0$.

\subsubsection{Weighted norm approach}

We propose an alternative misfit criterion, based on the weighted norm of the correlation:

$$
\phi_{W}=\|W \cdot C\|_{2}^{2} .
$$

This approach was introduced by the authors in the context of reflection tomography, or velocity analysis, of active seismic data (van Leeuwen \& Mulder, 2008a, 2010a). In appendix A we show that the weighted norm can be seen as a correlation of the squared weight with a regularizing kernel that depends on the source spectra:

$$
\phi_{W}(\Delta T) \simeq \int \mathrm{d} t W(t)^{2} \chi(t-\Delta T)
$$

where $\chi(t)=F(t)^{2}$. So, the location of the minimum, or maximum, of the functional does not depend directly on the behavior of $F$ anymore, as it did for the picking approach. This resembles techniques were the envelope of the correlation is used for picking, instead of the correlation itself (Gee \& Jordan, 1992). 

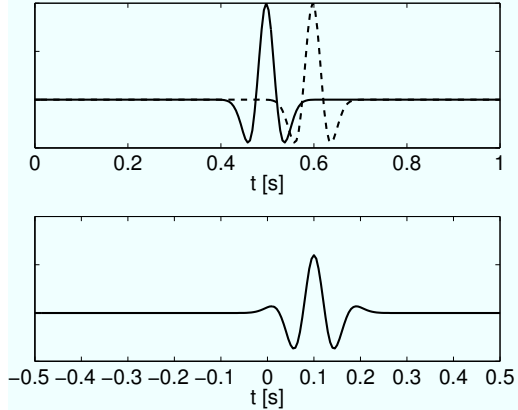

(a)

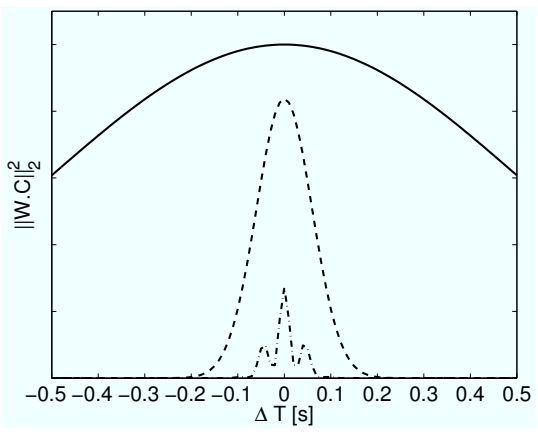

(c)

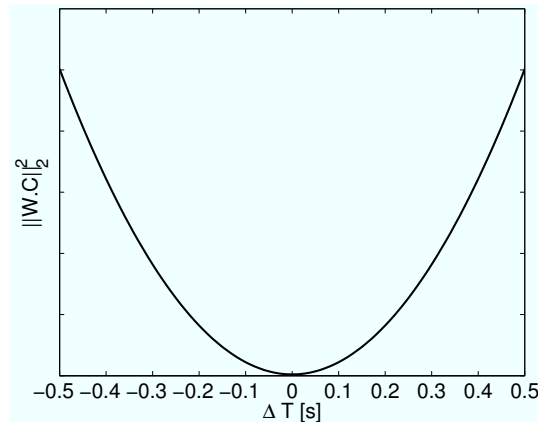

(b)

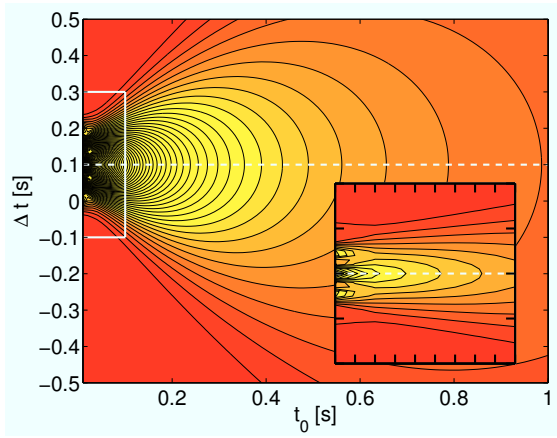

(d)

Figure 5.1. Example of different correlation-based misfit criteria. (a, top) shows two waveforms (Ricker wavelet with a $10 \mathrm{~Hz}$ peak frequency) that are phase shifted by $\Delta T=0.1 \mathrm{~s}$. The correlation of the waveforms is depicted in (a, bottom). The maximum of the correlation coincides with the phase shift. (b) depicts the weighted norm of the correlation using the linear weight (solid), as a function of the phase shift. The weighed norm using the Gaussian weight is depicted in (c) for $t_{0}=1 \mathrm{~s}$ (solid), $t_{0}=0.1 \mathrm{~s}$ (dash), and $t_{0}=0.01 \mathrm{~s}$ (dash-dot). The latter exhibits local maxima, indicating that $t_{0}$ is too small. The FBI transform of the correlation (a), $E_{\Delta t}$, as a function of $t_{0}$ and $\Delta t$ and a fixed frequency of $10 \mathrm{~Hz}$ (cf. eq 5.17) is depicted in (d). At $\Delta t=0.1 s E_{\Delta t}$ grows fastest as $t_{0} \rightarrow 0$, indicating the phase shift. For $t_{0}$ chosen too small, local maxima occur. This indicates the transition between the picking approach and the weighted norm with the Gaussian weight.

We investigate the behavior of the misfit functional as a function of the phase shift, $\Delta T$, in more detail for two specific choices of the weighting function:

$$
\begin{aligned}
& W_{t_{0}}^{(1)}(t)=\Omega_{t_{0}}\left(t_{0}\right) t, \\
& W_{t_{0}}^{(2)}(t)=\exp \left[-\left(t / t_{0}\right)^{2}\right],
\end{aligned}
$$

where the rectangle function $\Omega_{t_{0}}(t)=1$ when $\left|t / t_{0}\right| \leq 1$ and zero otherwise. The parameter $t_{0}$ controls the width of the weighting function and hence the maximal distance over which events are allowed to interact in the correlation. 

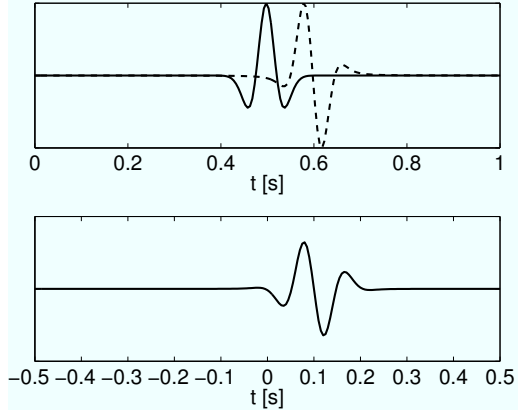

(a)

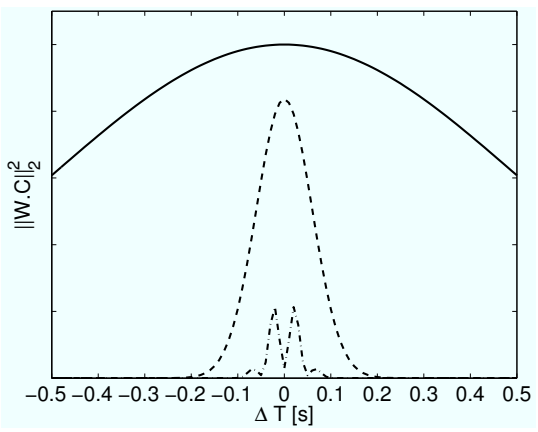

(c)

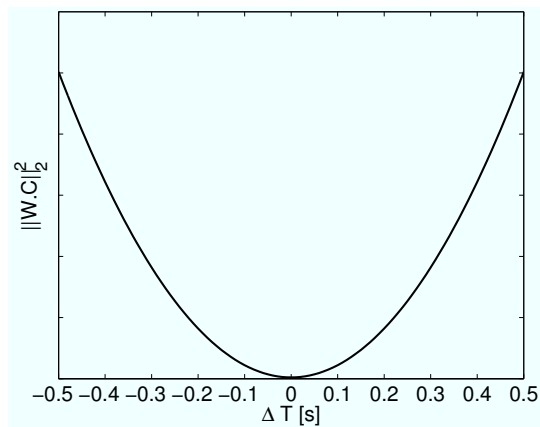

(b)

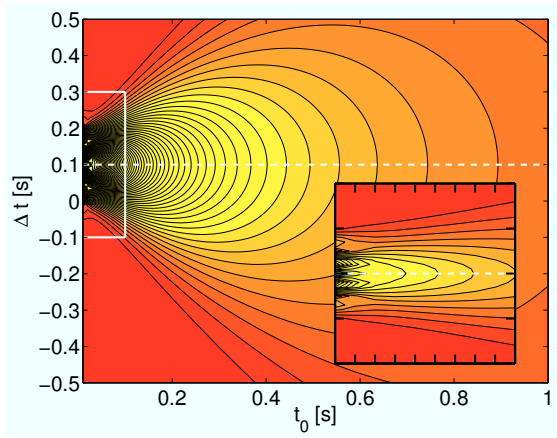

(d)

Figure 5.2. Example of different correlation-based misfit criteria. (a, top) shows two waveforms (Ricker wavelet with a $10 \mathrm{~Hz}$ peak frequency) that are phase shifted by $\Delta T=0.1 \mathrm{~s}$ and one of them has its phase rotated by $\pi / 2$. The correlation of the waveforms is depicted in (a, bottom). The maximum of the correlation does not coincide with the phase shift. Instead, the correlation has a zero-crossing at $t=0.1 \mathrm{~s}$. (b) depicts the weighted norm of the correlation (cf. eq 5.17) using the linear weight (solid), as a function of the phase shift. The weighed norm using the Gaussian weight is depicted in (c) for $t_{0}=1 \mathrm{~s}$ (solid), $t_{0}=0.1 \mathrm{~s}$ (dash), and $t_{0}=0.01 \mathrm{~s}$ (dash-dot). Again, the correlation and exhibits local maxima, indicating that $t_{0}$ is too small. The FBI transform of the correlation (a), $E_{\Delta t}$, as a function of $t_{0}$ and $\Delta t$ and a fixed frequency of $10 \mathrm{~Hz}$ (cf. eq 5.17) is depicted in (d). At $\Delta t=0.1 \mathrm{~s}, E_{\Delta t}$ grows fastest as $t_{0} \rightarrow 0$, indicating the phase shift. For $t_{0}$ chosen too small local maxima occur. This indicates the transition between the picking approach and the weighted norm with the Gaussian weight.

\section{Linear weight}

The linear weighting function, $W_{t_{0}}^{(1)}$, may be interpreted as an annihilator. It is readily verified that when the correlation can be represented as a shifted delta pulse, $C(t) \simeq \delta(t-\Delta T)$, the weighted norm is zero at $\Delta T=0$. The idea is to penalize energy at non-zero shift. By minimising, we are selecting a velocity that 

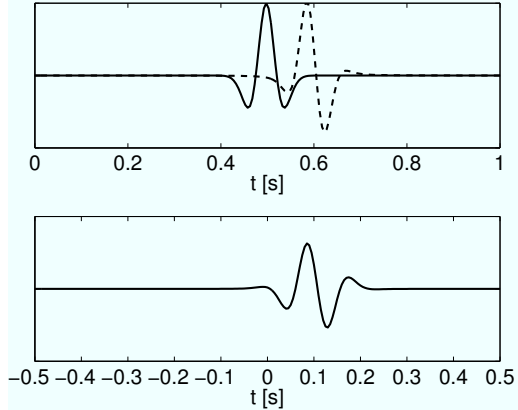

(a)

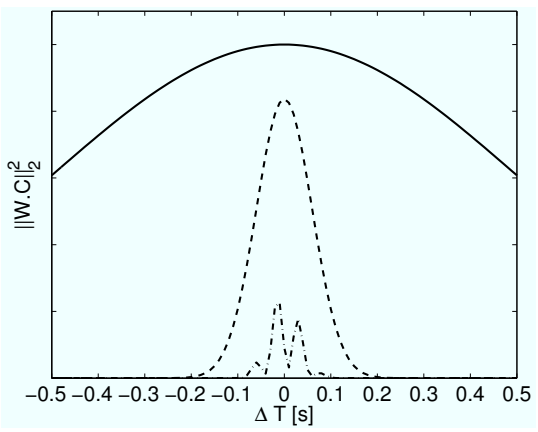

(c)

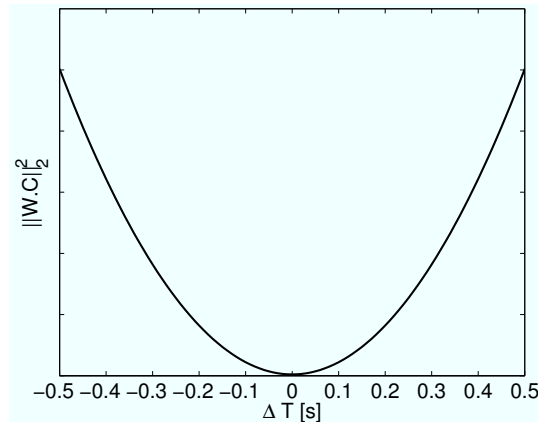

(b)

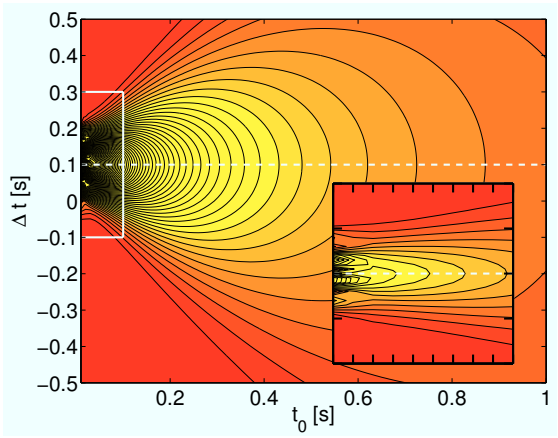

(d)

Figure 5.3. Example of different correlation-based misfit criteria. (a, top) shows two waveforms (Ricker wavelet with a $10 \mathrm{~Hz}$ peak frequency) that are phase shifted by $\Delta T=0.1 \mathrm{~s}$ and one of them has its phase rotated by $\pi / 3$. The correlation of the waveforms is depicted in (a, bottom). The maximum of the correlation does not coincide with the phase shift. (b) depicts the weighted norm of the correlation (cf. eq 5.17) using the linear weight (solid), as a function of the phase shift. The weighed norm using the Gaussian weight is depicted in (c) for $t_{0}=1 \mathrm{~s}$ (solid), $t_{0}=0.1 \mathrm{~s}$ (dash), and $t_{0}=0.01 \mathrm{~s}$ (dash-dot). Again, the latter exhibits local maxima, indicating that $t_{0}$ is too small. The FBI transform of the correlation, $E_{\Delta t}$, as a function of $t_{0}$ and $\Delta t$ and a fixed frequency of $10 \mathrm{~Hz}$ (cf. eq 5.17) is depicted in (d). At $\Delta t=0.1 \mathrm{~s}, E_{\Delta t}$ grows fastest as $t_{0} \rightarrow 0$, indicating the phase shift. For $t_{0}$ chosen too small local maxima occur. This indicates the transition between the picking approach and the weighted norm with the Gaussian weight.

will focus the energy in the correlation around zero shift. This leads to an approach that is conceptually the same as the annihilator-based approach to velocity analysis that has found widespread use among exploration geophysicists. We refer to Symes (2008b) for an excellent overview of these concepts.

Figs 5.1-5.3 (b) illustrate the properties of the misfit functional as a function of the phase shift, $\Delta T$, for three different scenarios: the source spectra are identical, phase rotated over $\pi / 2$ or phase rotated over $\pi / 3$. In all cases the misfit attains a 
minimum at $\Delta T=0$ and is quadratic in $\Delta T$.

Under the assumption that $\chi$ is even (this includes phase rotation over $\pi / 2$ or a factor $\imath \omega$ between the source spectra), we show in Appendix A that

$$
\phi_{W_{t_{0}}^{(1)}}(\Delta T) \simeq\left\|W_{t_{0}}^{(1)} \cdot F\right\|_{2}^{2}+(\Delta T)^{2}\|F\|_{2}^{2},
$$

which confirms the quadratic dependency of the misfit on $\Delta T$. This indicates that there may be more to this approach than is suggested in the annihilator framework, which deals with the propagation of singularities rather than finite-frequency waveforms (cf. Stolk \& de Hoop, 2006). Having made this remark, we leave the matter for further research.

\section{Gaussian weight}

The Gaussian weighting function does not act as an annihilator. Instead, the misfit functional attains a maximum at $\Delta T=0$ (see appendix A). Figs 5.1-5.3 (c) illustrate this. The picking approach, as explained in section 3.1, may be derived from this approach by letting $t_{0} \downarrow 0$. The Gaussian weight then collapses to a delta function and maximizing the weighted norm is equivalent to maximizing the correlation at zero shift. This can be seen from figs 5.1-5.3(c,d), where the Gaussian-weighted norm develops local maxima for very small $t_{0}$.

A tentative link may be made with an approach discussed by Hörmann \& de Hoop (2002) (see also de Hoop \& van der Hilst, 2005). They propose to detect the phase shift by a time-frequency analysis of the correlation as follows. Consider the windowed Fourier transform of the correlation:

$$
E_{\Delta t}\left(t_{0}, \omega\right)=\int \mathrm{d} t W_{t_{0}}^{(2)}(t-\Delta t) C(t) \exp [-\imath \omega t] .
$$

This is also known as the FBI (Fourier-Bros-Iagolnitzer) or Gabor transform (cf. Folland, 1989; Strichartz, 2003). The singular support of the correlation (i.e., the location of the singularity) may be detected by inspecting the growth properties of $E_{\Delta t}\left(t_{0}, \omega\right)$ as $t_{0} \downarrow 0$. More precisely, when $\Delta t$ is not in the singular support of the correlation we have, for any $N \in \mathbb{N}$,

$$
\left|E_{\Delta t}\left(t_{0}, \omega\right)\right| \leq c_{N}\left(t_{0}\right)^{N} .
$$

(cf. Hörmann \& de Hoop, 2002). So when $\Delta t$ does not coincide with the singular support of the correlation, $E_{\Delta t}\left(t_{0}, \omega\right)$ should be rapidly decreasing as $t_{0} \downarrow 0$. By inspecting the graph of $E_{\Delta t}\left(t_{0}, \omega\right)$ as a function of $t_{0}$ for each $\Delta t$ and a fixed $\omega$ we should select the $\Delta t$ for which $E_{\Delta t}\left(t_{0}, \omega\right)$ grows fastest as $t_{0} \downarrow 0$. This is clearly illustrated in figs $5.1-5.3$ (d). Again, for very small $t_{0}$ this approach reduces to the picking approach.

The weighted norm approach arises when we consider $E_{\Delta t}$ for a fixed $t_{0}$ and all frequencies. In fact, it is readily verified that

$$
\left\|E_{0}\left(t_{0}, \cdot\right)\right\|_{2}^{2}=\left\|W_{t_{0}}^{(2)} C\right\|_{2}^{2}
$$

which in light of the above seems a reasonable way to measure the shift of the singular support. 


\subsection{Sensitivity kernels}

The sensitivity kernel relates perturbations in the medium parameters to changes in the misfit. It should be noted that the sensitivity kernel only gives a meaningful update of the model if it is consistent with the misfit (de Hoop \& van der Hilst, 2005). Although the derivation of such kernels is rather straight-forward we include it for completeness' sake.

Via a Taylor-series argument we have:

$$
\phi\left[c_{0}+\delta c\right]-\phi\left[c_{0}\right]=\int \mathrm{d} \mathbf{x} \partial_{c} \phi(\mathbf{x}) \delta c(\mathbf{x})+\mathcal{O}\left(\|\delta c\|_{2}^{2}\right),
$$

Where $\partial_{c} \phi(\mathbf{x})$ is the sensitivity kernel, or the Fréchet derivative of the misfit functional w.r.t. to the medium parameters. In the context of transmitted body waves, one usually employs the Born approximation to derive this kernel, which can then be efficiently computed by correlating a forward and adjoint wavefield (see Tarantola, 1984, for example). We briefly illustrate this procedure with an example. Concentrating on a single component, one source-receiver pair and a scalar medium perturbation, we assume that the wavefield obeys a scalar wave equation:

$$
\underbrace{\left[c^{-2} \partial_{t}^{2}-\nabla^{2}\right]}_{\mathcal{L}[c]} u=f \delta\left(\cdot-\mathbf{x}_{s}\right) .
$$

We apply a Born approximation, writing $c=c_{0}+\delta c, u=u_{0}+\delta u$ :

$$
\begin{aligned}
\mathcal{L}\left[c_{0}\right] u_{0}(t, \mathbf{x}) & =f \delta\left(\mathbf{x}-\mathbf{x}_{s}\right) \\
\mathcal{L}\left[c_{0}\right] \delta u(t, \mathbf{x}) & =2 \frac{\delta c(\mathbf{x})}{c_{0}(\mathbf{x})^{3}} \partial_{t}^{2} u_{0}(t, \mathbf{x}) .
\end{aligned}
$$

We also introduce the wavefield $v_{0}$ satisfying

$$
\mathcal{L}^{*}\left[c_{0}\right] v_{0}=\partial_{d} \phi(t) \delta\left(\mathbf{x}-\mathbf{x}_{r}\right),
$$

where $\partial_{d} \phi$ is the Fréchet derivative of the misfit functional w.r.t. to the data, or the adjoint source. The sensitivity kernel is then given by

$$
\partial_{c} \phi(\mathbf{x})=\frac{2}{c_{0}(\mathbf{x})^{3}} \int \mathrm{d} t v_{0}(t, \mathbf{x}) \partial_{t}^{2} u_{0}(t, \mathbf{x}) .
$$

For more details concerning the adjoint-state technique we refer to Plessix (2006). Asymptotically, the kernel is then given by (dropping the factor $2 c_{0}^{-3}$ ):

$$
\partial_{c} \phi(\mathbf{x}) \simeq a_{s} a_{r} \int \mathrm{d} \omega \widehat{\partial_{d} \phi}(\omega) \omega^{2} f^{*}(\omega) \exp \left[\imath \omega\left(T_{s}+T_{r}\right)\right]
$$

We derive the adjoint sources for the different correlation-based approaches in appendix B. This yields the following expressions for the sensitivity kernels:

$$
\partial_{c} \phi(\mathbf{x})=a_{s} a_{r} \int \mathrm{d} \omega \omega^{2} \hat{F}(\omega) \hat{H}(\omega) \exp \left[\imath \omega\left(T_{s}+T_{r}-T\right)\right]
$$




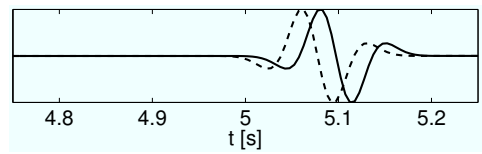

(a)

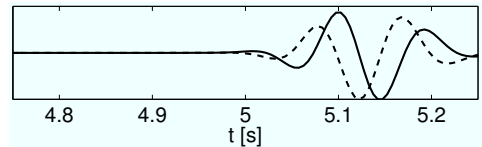

(b)

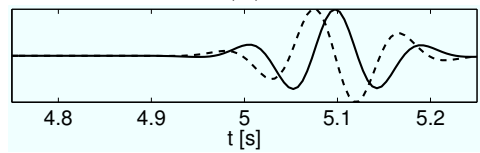

(c)

Figure 5.4. Example of adjoint sources for the different correlation-based approaches: (a) picking, (b) linear weight and (c) Gaussian weight for $t_{0}=1$. The waveforms are phase-shifted by $0.1 \mathrm{~s}$ and their spectra are either the same (solid) or phase-rotated over $\pi / 2$ (dash) w.r.t. each other. The shift of the adjoint sources for the picking approach depends on the source spectra. For the weighted norm approach, the phase rotation of the source spectrum causes a phase rotation of the adjoint source.

The factor $\hat{H}$ depends on the misfit criterion, which for source signatures that are band-limited delta functions is given by:

$$
\begin{aligned}
\hat{H}_{\max }(\omega) & \simeq \text { (amplitude terms }) \times \imath \omega \Delta T \exp \left[\imath \omega \epsilon_{T}\right], \\
\hat{H}_{W}(\omega) & \simeq \text { (amplitude terms }) \times W(\Delta T)^{2} .
\end{aligned}
$$

When $\hat{F}$ is real and the velocity varies only mildly, eq. 5.27 leads to the famous banana-doughnut shaped kernel with zero sensitivity along the central ray (Marquering et al., 1999). When $\hat{F}$ is not real, which happens in general when the source spectra are not identical, the shape of the kernel changes and loses the zero-sensitivity along the ray. This is a subtle, but important, point first made by de Hoop \& van der Hilst (2005).

The kernel for the weighted norm approach does not have zero-sensitivity along the ray. For general source spectra, we find that the factor $\hat{H}_{W}$ contains a factor $\hat{F}^{*}$. Under the assumption that $\chi$ is an even function, the factor $\hat{F} \hat{H}_{W}$ (cf. eq 5.27) is real and even and defines an even function around the central ray. Therefore, the sensitivity kernel for the weighted norm approach has non-zero sensitivity along the central ray, as long as $\chi$ is even. We might call these kernels bananas instead of banana-doughnuts. This confirms our earlier assertion that the weighted-norm approach is less sensitive to differences in the source spectra. A similar, more detailed multi-resolution analysis of the sensitivity kernel for the picking approach is given by de Hoop \& van der Hilst (2005). 
To illustrate the above mentioned properties, we calculate the adjoint sources for the different approaches for two different scenarios: 1) the source spectra are identical and 2) the source spectra are phase rotated over $\pi / 2$ w.r.t. each other. The different adjoint sources are depicted in fig 5.4. As predicted, the phase rotation leads to a phase shift in the adjoint source for the picking approach. The adjoint sources for the weighted-norm approach respond to the phase rotation of the source spectrum by phase rotation, as argued above.

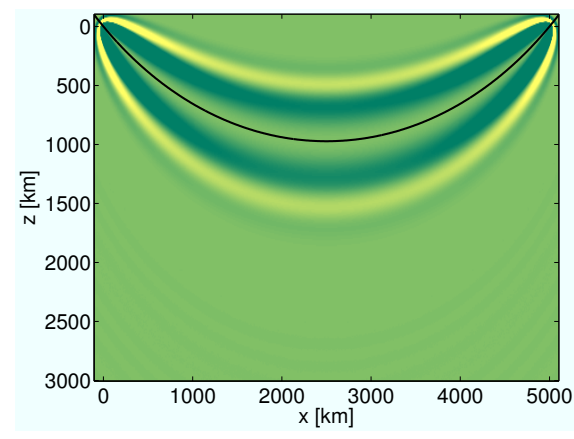

(a)

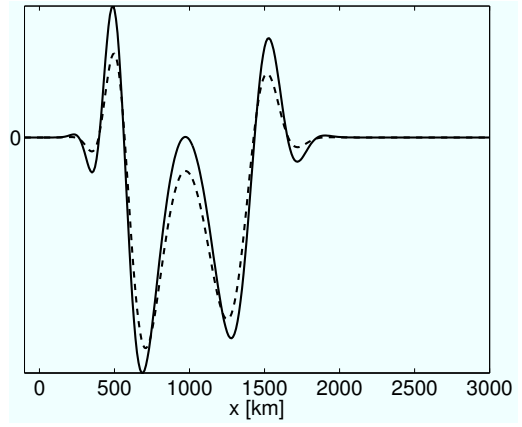

(c)

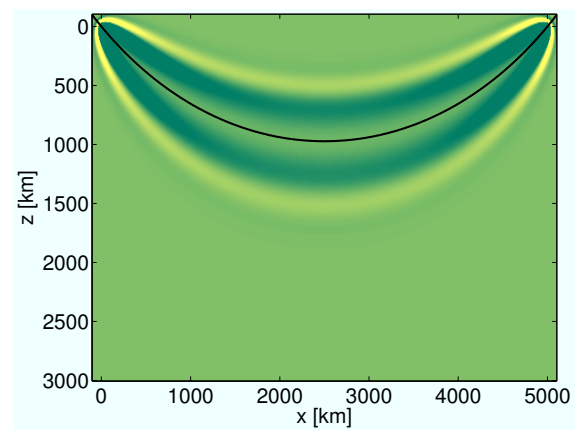

(b)

Figure 5.5. Example of sensitivity kernels for the picking approach for a velocity that increases linearly with depth. (a) and (c, solid) depicts the kernel where the source spectra are identical, while (b) and (c, dash) depicts a situation where the source spectra are phase rotated over $\pi / 2$. The slice through the kernels at $x=2500 \mathrm{~m}$ shows that kernel has zero sensitivity on the central ray when the source spectra are identical and non-zero sensitivity when the source spectra are not identical. 


\subsection{Example: diving wave tomography}

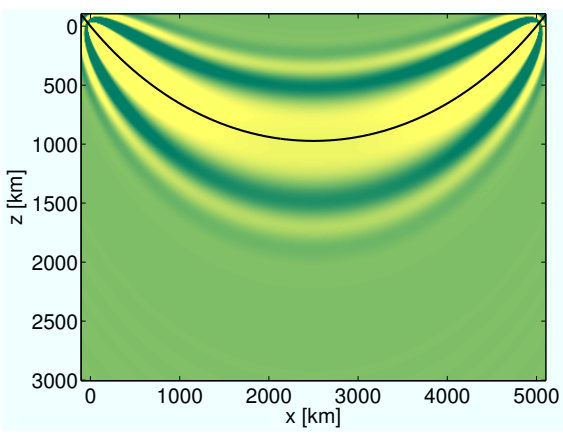

(a)

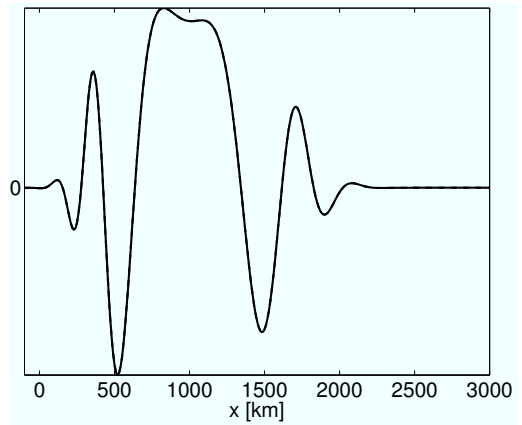

(c)

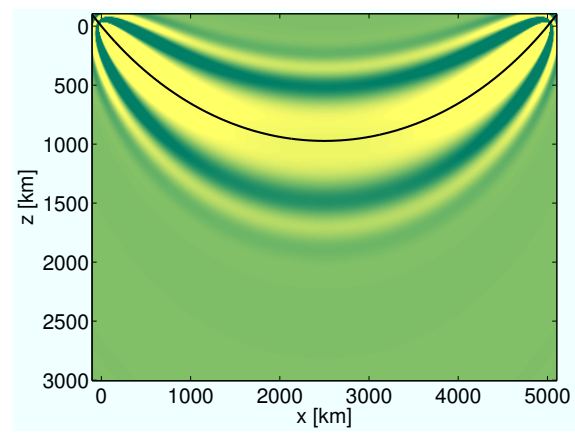

(b)

Figure 5.6. Example of sensitivity kernels for the weighted norm approach with the linear weight. (a) and (c, solid) depicts the kernel where the source spectra are identical, while (b) and (c, dash) depicts a situation where the source spectra are phase rotated over $\pi / 2$. The kernel is not affected by the phase-rotation.

We illustrate the properties of the sensitivity kernels derived in the previous section for constant-density acoustic velocity models that increase linearly with depth:

$$
c\left(x_{3}\right)=c_{0}+\alpha x_{3} .
$$

The 3-D Green's function for such models can be expressed analytically (Pekeris, 1946; Kuvshinov \& Mulder, 2006) and is given by

$$
G\left(\omega, \mathbf{x}, \mathbf{x}^{\prime}\right)=\left(4 \pi \sqrt{c c_{0}\left(\rho^{2}-1\right) / \alpha}\right)^{-1} \exp \left[\imath \sqrt{(\omega / \alpha)^{2}-1 / 4} \operatorname{arccosh}(\rho)\right]
$$

where

$$
\rho=1+\frac{1}{2} \frac{\left\|\mathbf{x}-\mathbf{x}^{\prime}\right\|_{2}}{\left(x_{3}+c_{0} / \alpha\right)\left(x_{3}^{\prime}+c_{0} / \alpha\right)} .
$$




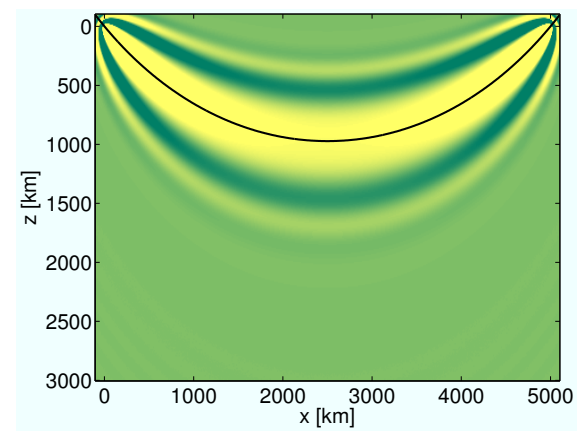

(a)

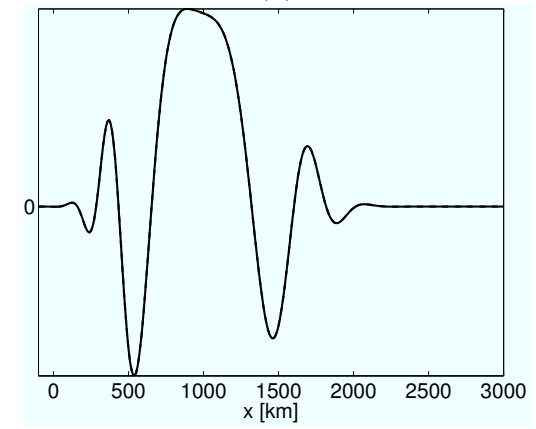

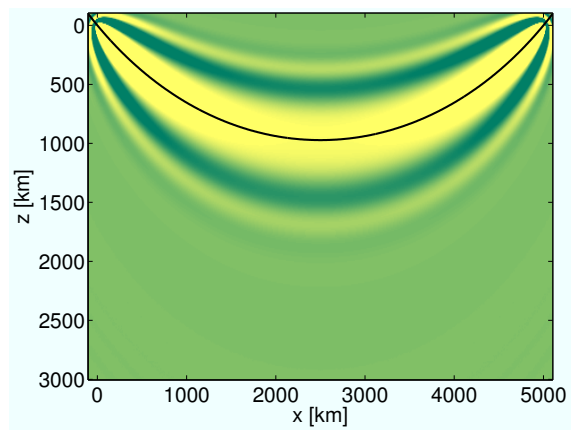

(b)

(c)

Figure 5.7. Example of sensitivity kernels for the weighted norm approach with the Gaussian weight. (a) and (c, solid) depicts the kernel where the source spectra are identical, while (b) and (c, dash) depicts a situation where the source spectra are phase rotated over $\pi / 2$. The kernel is not affected by the phase-rotation.

The kernel is explicitly given by

$$
\partial_{c} \phi\left(\mathbf{x}, \mathbf{x}_{s}, \mathbf{x}_{r}\right)=\int \mathrm{d} \omega \omega^{2} f^{*}(\omega) \widehat{\partial_{d} \phi}\left(\omega, \mathbf{x}_{s}, \mathbf{x}_{r}\right) G\left(\omega, \mathbf{x}_{s}, \mathbf{x}\right)^{*} G\left(\omega, \mathbf{x}, \mathbf{x}_{r}\right)^{*}
$$

Figs 5.5-5.6 illustrate the different kernels for $c_{0}=\bar{c}_{0}=1500$ and $\alpha=0.7 \mathrm{~s}^{-1}$, $\bar{\alpha}=0.5 \mathrm{~s}^{-1}$, again for identical and phase-rotated source spectra. The central ray is also indicated. The kernel for the picking approach is affected by the phase rotation of the source spectra, while the weighted norm kernels are visibly indiscernible, confirming our earlier assertion.

\subsection{Application: cross-well tomography}

We use the weighted norm of the correlation with the Gaussian weight as a misfit criterion for cross-well tomography. That is, we solve the optimisation problem

$$
c_{\text {optimal }}=\underset{c}{\operatorname{argmax}} \phi_{W_{t_{0}}^{(2)}}[c] .
$$




\begin{tabular}{c|c|c|c|c|c} 
& $\gamma$ & $t_{r}$ & $Q_{r}$ & $f_{r}$ & $c_{0}$ \\
\hline $\mathrm{P}$ & 8 & 0.05 & 80 & 100 & 1551 \\
$\mathrm{~S}$ & 4 & 0.1 & 80 & 100 & 430
\end{tabular}

Table 5.1. Inversion parameters for $P$ and $S$ velocity

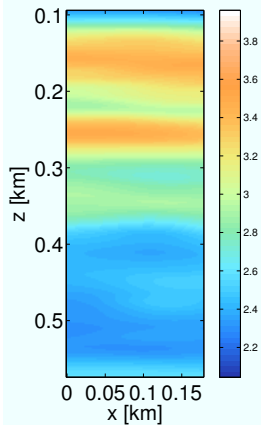

(a)

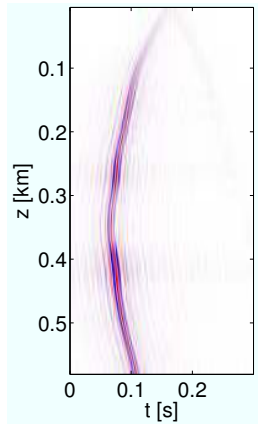

(d)

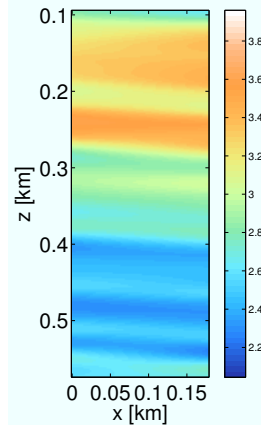

(b)

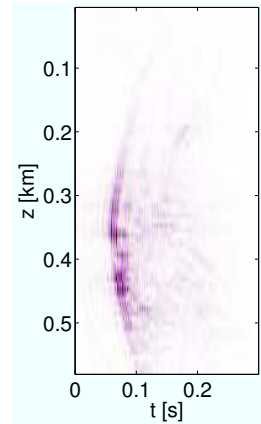

(e)

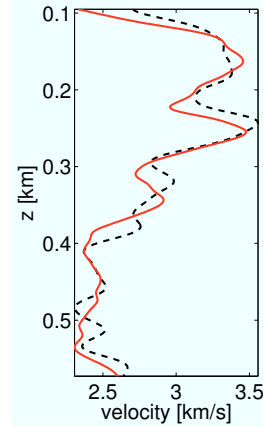

(c)

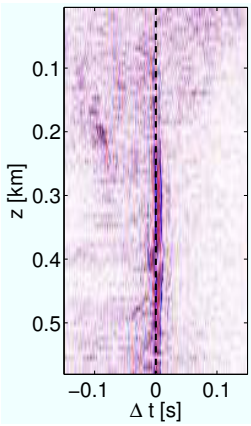

(f)

Figure 5.8. Inversion of the $x$-component of cross-well data for an $x$ source using the weighted norm of the correlation as optimisation criterion. We used the Gaussian as weighting function. (a) depicts the final velocity, (b) depicts the interpolated and smoothed well-logs. (c) depicts a central slice through the final (solid) and well-log (dash) models. (d) and (e) show a shotgather at $387.4 \mathrm{~m}$ of the modelled and observed data. The correlation of the latter two is depicted in (f). To allow for visual inspection, the correlation is normalized by dividing by the spectrum.

The data are modelled with a frequency-domain finite-difference code (Mulder \& Plessix, 2002). We employ a limited-memory BFGS (Broyden-Fletcher-ShannoGoldfarb) method to solve the optimisation problem. As regularisation, we represent the model on linear splines on a coarse grid. The gradient is calculated with the adjoint-state technique. More details on how to implement the adjoint calculations can be found in (Tromp et al., 2005; Plessix, 2006, for example).

The cross-well data set, which was also use for a virtual source study (Mehta et al., 


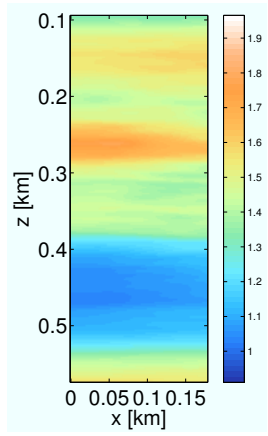

(a)

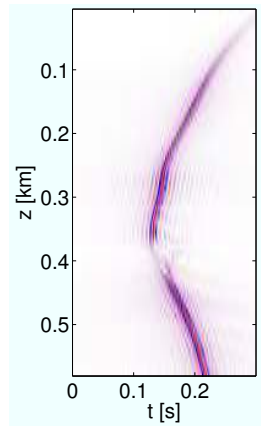

(d)

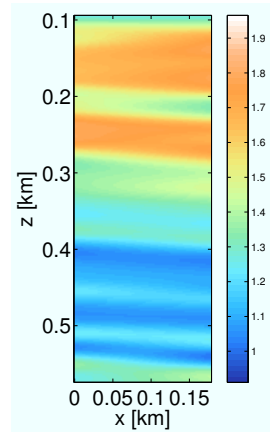

(b)

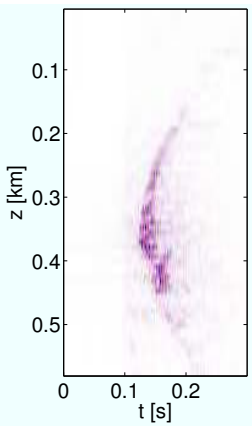

(e)

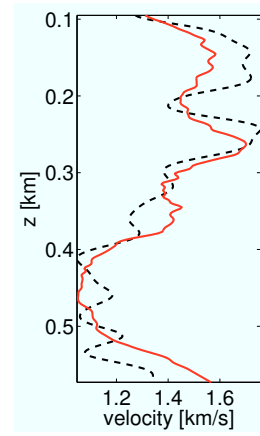

(c)

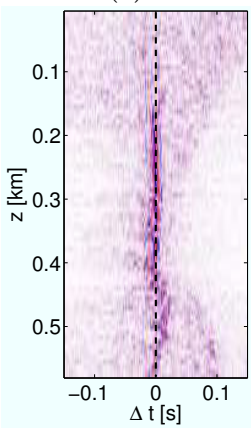

(f)

Figure 5.9. Inversion of the $y$-component of cross-well data for a $y$-source using the weighted norm of the correlation as optimisation criterion. We used the Gaussian as weighting function. (a) depicts the final velocity, (b) depicts the interpolated and smoothed well-logs. (c) depicts a central slice through the final (solid) and well-log (dash) models. (d) and (e) show a shotgather at $387.4 \mathrm{~m}$ of the modelled and observed data. The correlation of the latter two is depicted in ( $f$ ). To allow for visual inspection, the correlation is normalized by dividing by the spectrum.

2008), contains 147 shot positions in the well at $x=205.74 \mathrm{~m}$ between $7.19 \mathrm{~m}$ and $567.9 \mathrm{~m}$ depth at a $3.84 \mathrm{~m}$ interval and 150 receiver positions at $x=26.82 \mathrm{~m}$ between $6.63 \mathrm{~m}$ and $578.86 \mathrm{~m}$ depth at the same depth interval. The source and $3 \mathrm{C}$ geophones were clamped to the casing.

We used the $x$-component of the data for an $x$-source to estimate the $\mathrm{P}$ velocity and the $y$-component of the data for a $y$-source to estimate the $\mathrm{S}$ velocity separately. We assumed that the separate components were not too different from acoustic pressure data from an explosive source. The data where damped to emphasise the first arrival by multiplication with $e^{-\gamma\left(t-t_{r}\right)}$. This translates into a complex angular frequency with $\gamma$ as its imaginary part. We inverted frequencies from 70 to $160 \mathrm{~Hz}$ with an interval of $2 \mathrm{~Hz}$. Attenuation is taken into account: $\tilde{c}=c / \sqrt{1+(\imath-a) / Q}, a=(2 / \pi) \log \left(f / f_{r}\right)$. The free surface was also modelled by imposing a zero-pressure boundary condition at the top of the model. As a wavelet 
we used a band-limited delta function.

The inversion was done in several stages. First, an optimal (i.e., resulting in the lowest misfit) linear velocity $c=c_{0}+\alpha x_{3}$ was sought at a fixed quality factor $Q=Q_{r}$. Then, we inverted for a layered model, represented by linear splines at a $15.24 \mathrm{~m}$ interval, again at a fixed $Q=Q_{r}$. Finally, we allowed $Q$ to vary and used a finer spline grid to represent the velocity. The inversion parameters for the $\mathrm{P}$ and $\mathrm{S}$ velocity are given in table 5.1.

The final results are shown in fig 5.8 and 5.9. We compare the final models with the interpolated well-logs. This shows that the general trend of the velocity matches pretty well. Also, the traveltimes are reasonably well explained, as can be seen from the correlation panels. To allow for visual inspection of the correlation panels we normalized the correlation by dividing by the absolute value of the spectrum.

A perfect match, in terms of velocity and traveltime, is not be expected since important effects such as anisotropy have not been taken into account. Also, the damping parameters used do probably not reflect the reality, which may seriously effect the result. We have generated synthetic data for the interpolated well-logs and these data did not give a significantly better traveltime match than our inverted model. This indicates that the resulting traveltime and velocity errors are most probably caused by systematic modelling errors. We did not try to adjust the damping parameters or introduce anisotropy to improve the results, as this example serves as a proof of concept of the method.

\subsection{Discussion}

The weighted norm of the correlation can be seen as a regularized version of the correlation and resembles techniques that use the envelope or a time-frequency analysis of the correlation to pick the phase shift. The main difference with such approaches is that the weighted norm can be used directly as an optimisation criterion, without picking. In particular, this makes it easier to derive the corresponding sensitivity kernels, as we do not need to use implicit relations between the maximum of the correlation and the phase shift.

The behavior of the sensitivity kernel for the picking approach is determined by the correlation of the source spectra, $\hat{F}(\omega)=\bar{f}(\omega) f^{*}(\omega)$. If, for example, the source spectra differ by a phase rotation, this will show up in $\hat{F}$ and hence directly in the kernel, causing the zero-sensitivity along the central ray to disappear. In the sensitivity kernels for the weighted norm approach an extra factor $\hat{F}^{*}$ appears, making these kernels less sensitive to errors in the source spectra. For example, phase rotation or differentiation of the source spectrum will not dramatically affect the weighted norm approach. The link to the time-frequency analysis, presented in section 3, suggests that the weighting function plays a vital role in mitigating this sensitivity. If we choose the width of the Gaussian weight too small, the weighted norm degrades to the picking approach. If we choose it too large, we might not be sensitive enough to time shifts. The width of the Gaussian should somehow reflect the frequency content of the data. One might even envision a scheme were different frequency bands of the data are treated separately with an optimal width of the 
Gaussian.

Intuitively, the weighted norm approach should be able to handle multiple arrivals at the same time, as long as each arrival in the observed data has a corresponding arrival in the modelled data. An issue that arises here is the cross-talk between different arrivals. Moreover, the correlation could be extended to measure phase differences of whole wavefields. Then, not only the shift of the singular support but also the slowness difference between the wavefields can be measured. This would allow for a wave-equation stereo-tomography approach.

\subsection{Conclusion}

We have reviewed several correlation-based misfit functionals that are sensitive to the time shift between complex waveforms. Such misfit functionals are used to cast wave-equation traveltime tomography as a PDE-constrained optimisation problem. Ideally, the misfit functional attains a minimum - or maximum - if the phase shift between the measured and observed data is zero. The commonly-used procedure that relies on picking the maximum of the correlation has the drawback that it is very sensitive to errors in the estimated source signature. When the modeled and observed source spectra are not identical, the corresponding misfit functional may attain its minimum at non-zero phase shift. As an alternative to the picking approach, we propose to use a weighted norm of the correlation as misfit functional. We discuss two particular instances of this approach, using a linear and a Gaussian weighting function. For the linear weighting function, we may view the procedure as an annihilator-based criterion which attains a minimum when the phase shift is zero. Using the Gaussian weight, the weighted norm attains a maximum. We show that this approach is intimately connect to techniques from time-frequency analysis. The sensitivity kernels corresponding to the weighted norm approach do not exhibit the characteristic hole in the center and are less sensitive to errors in the source spectra. One might say that these kernels look more like regular bananas instead of banana-donuts. We illustrate the properties of the sensitivity kernels for a velocity model that increase linearly with depth, using an analytic solution of the constantdensity acoustic wave-equation.

The cross-well examples illustrate that the weighted norm approach is a viable alternative to the picking approach which is more robust w.r.t. errors in the estimated source spectrum.

\subsection{Appendix A: Weighted norm of the correlation}

In the following we will drop the amplitude and source-receiver dependence from the notation, expressing the data as

$$
d(t)=(2 \pi)^{-1} \int \mathrm{d} \omega f(\omega) \exp [\imath \omega(t-T)]
$$


The correlation is then given by

$$
\begin{aligned}
C(t) & =(2 \pi)^{-1} \int \mathrm{d} \omega \hat{F}(\omega) \exp [\imath \omega(t-\Delta T)] \\
& =F(t-\Delta T)
\end{aligned}
$$

where $\hat{F}=\bar{f} f^{*}$ is the correlation of the source spectra. The weighted norm of the correlation, as a function of the phase shift, is then given by

$$
\begin{aligned}
\phi_{W}(\Delta T) & =\int \mathrm{d} t(W(t) F(t-\Delta T))^{2} \\
& =\int \mathrm{d} t B(t+\Delta T) \chi(t)
\end{aligned}
$$

where $B=W^{2}$ and $\chi=F^{2}$. The behavior of the functional, as a function of $\Delta T$ depends intricately on the interplay between the weighting function and the correlation of the source spectra. In the following we assume that $\chi$ is an even function. This restricts the validity of the analysis to cases where $\hat{F}$ is either purely real or purely imaginary (this includes the source spectra differing by a phase rotation over $\pi / 2$ degrees or a factor $\imath \omega)$. The example in fig 5.3 suggests, however, that the validity of the approach is not restricted to this case. Also, the more general result on the detection of wavefront sets by using the Gaussian weight (Hörmann \& de Hoop, 2002) suggests a more general validity. The following analysis is meant to illustrate why the weighted norm approach might work.

We investigate the behavior of the misfit functional as a function of $\Delta T$. The first derivate is given by

$$
\phi_{W}^{\prime}(\Delta T)=\int \mathrm{d} t B^{\prime}(t+\Delta T) \chi(t)
$$

Since $\chi$ is even and $B^{\prime}$ is odd, $\phi_{W}^{\prime}(0)=0$. For the specific cases $B(t)=t^{2}$ (linear weight) and $B(t)=e^{-2 t^{2}}$ (Gaussian weight), it is readily verified that $\Delta T=0$ is the only stationary point of $\phi_{W}^{\prime}$. The second derivative is given by

$$
\phi_{W}^{\prime \prime}(\Delta T)=\int \mathrm{d} t B^{\prime \prime}(t+\Delta T) \chi(t) .
$$

For the linear weight, $B^{\prime \prime}(0)=2$, so $\phi_{W}^{\prime \prime}(0)>0$ i.e., the misfit functional has a minimum at $\Delta T=0$. Likewise, we find that the misfit functional has a maximum for the Gaussian weight.

For the linear weight we can derive an even more explicit expression:

$$
\begin{aligned}
\phi_{W^{(1)}}(\Delta T) & =\int \mathrm{d} t(t+\Delta T)^{2} \chi(t) \\
& =(\Delta T)^{2}\|F\|_{2}^{2}+2 \Delta T \int \mathrm{d} t t \chi(t)+\left\|W^{(1)} \cdot F\right\|_{2}^{2} .
\end{aligned}
$$

The second term vanishes because the integrand is odd. 


\subsection{Appendix B: Sensitivity kernels}

The sensitivity kernel is given by

$$
\partial_{c} \phi \simeq \int \mathrm{d} \omega \omega^{2} f^{*}(\omega) \widehat{\partial_{d} \phi}(\omega) \exp \left[\imath \omega\left(T_{s}+T_{r}\right)\right]
$$

The adjoint sources for the different functionals are given by :

$$
\begin{aligned}
\partial_{d} \phi_{\max }(t) & =-2 \Delta t \frac{\partial_{t} \bar{d}(t+\Delta t)}{C\left[\partial_{t}^{2} \bar{d}, d\right](\Delta t)}, \\
\partial_{d} \phi_{W}(t) & =\int \mathrm{d} t^{\prime} W\left(t^{\prime}\right)^{2} C\left(t^{\prime}\right) \bar{d}\left(t^{\prime}+t\right) .
\end{aligned}
$$

For a derivation of eq (5.43) we refer to (Luo \& Schuster, 1991).

Upon substituting the asymptotic expression for the data in eq (5.43), we get

$$
{\widehat{\partial_{d} \phi}}_{\max }(\omega) \simeq \frac{2\left(\Delta T+\epsilon_{T}\right)}{C\left[\partial_{t}^{2} \bar{d}, d\right](\Delta t)} \imath \omega \bar{f}(\omega) \exp \left[\imath \omega\left(\epsilon_{T}-T\right)\right] .
$$

This leads to the kernel for the picking approach:

$$
\partial_{c} \phi_{\max }=\frac{2\left(\Delta T+\epsilon_{T}\right)}{C\left[\partial_{t}^{2} \bar{d}, d\right](\Delta t)} \int \mathrm{d} \omega \imath \omega^{3} \hat{F}(\omega) \exp \left[\imath \omega\left(T_{s}+T_{r}-T+\epsilon_{T}\right)\right] .
$$

For the weighted-norm approach we find, by inserting the asymptotic expressions for the data into eq (5.44) and taking the Fourier transform:

$$
\begin{aligned}
\widehat{\partial d}_{W}(\omega) & \simeq \int \mathrm{d} t \int \mathrm{d} t^{\prime} \int \mathrm{d} \omega^{\prime} \int \mathrm{d} \omega^{\prime \prime} \hat{F}\left(\omega^{\prime}\right) \bar{f}\left(\omega^{\prime \prime}\right) B\left(t^{\prime}\right) \\
& \times \exp \left[-\imath \omega t+\imath \omega^{\prime}\left(t^{\prime}-\Delta T\right)+\imath \omega^{\prime \prime}\left(t^{\prime}+t-\bar{T}\right)\right]
\end{aligned}
$$

In case the source spectra are band-limited delta functions, the integrals can be collapsed directly to yield:

$$
\widehat{\partial} d_{W}(\omega) \simeq B(\Delta T) \exp [-\imath \omega T]
$$

which leads to

$$
\partial_{c} \phi_{W} \simeq B(\Delta T) \int \mathrm{d} \omega \exp \left[\imath \omega\left(T_{s}+T_{r}-T\right)\right]
$$

For general source spectra, we may insert the expression into eq (5.42). Rewriting in terms of the Fourier transform of $B$, we get:

$$
\begin{aligned}
\partial_{c} \phi_{W} \simeq & \int \mathrm{d} \omega \int \mathrm{d} \omega^{\prime} \omega^{2} \hat{F}(\omega) \hat{F}^{*}\left(\omega^{\prime}\right) \hat{B}\left(\omega^{\prime}-\omega\right) \\
& \times \exp \left[\imath \omega\left(T_{s}+T_{r}-T\right)+\imath\left(\omega^{\prime}-\omega\right)(\Delta T)\right] .
\end{aligned}
$$




\title{
6 \\ Correlation-based \\ wave-equation reflection tomography
}

\begin{abstract}
We discuss and illustrate the application of the correlation-based misfit functional to wave-equation reflection tomography. This extension is important because it would eventually allow for application of the method to reflection data from geologically complex areas. The main difference with the previous application to NMO velocity analysis (chapter 2) and transmission tomography (chapter 4) is that we need to determine the reflectors as part of the process. A natural framework for this is the Born approximation. Least-squares estimation of the reflectivity then leads to a projection operator which projects the data onto the range of the modelling operator. The data and their projection will have different traveltimes at non-zero offset when the velocity is not correct. The correlation-based misfit criteria may then be applied to measure this difference in traveltimes.
\end{abstract}




\subsection{Introduction}

While the processing of seismic data still relies heavily on raytracing and picking of traveltimes, recent developments are moving towards full-waveform processing of the data. By this we mean using the full two-way wave equation for migration and velocity analysis. In the early 80's Tarantola pioneered this approach by formulating a PDE-constrained data-fitting problem known as waveform inversion. While it is in principle possible to include any relevant physics in the modelling it soon became apparent that the huge computational expenses involved did not deliver the results it promised. Most notably, a very good - kinematically correct - starting model is required to get a decent result. Recently, however, waveform inversion is gaining in popularity for a couple of reasons. Firstly, both hardware and algorithmic developments have made full waveform modelling more accessible. Secondly, new datasets with low frequencies and long offsets are better suited for waveform inversion, and thirdly, it has been recognized that two-way wave-equation migration (reverse time migration, RTM) can outperform one-way wave equation migration methods in the presence of strong lateral velocity variations.

However, it is still common practice to start waveform inversion from an initial model obtained by classical velocity analysis that is based on ray-tracing or oneway wave equations. This motivates the quest for a velocity analysis method that is based on the full, two-way wave equation. One such development is based on focussing an image as a function of depth-offset (Doherty \& Claerbout, 1974; Sava \& Biondi, 2004; de Hoop et al., 2006; Shen \& Symes, 2008), but uses RTM as migration kernel (Mulder \& van Leeuwen, 2008). While this method is based on the Born approximation, a non-linear generalization has been recently proposed by Symes (2008a).

In this chapter we propose an alternative method for velocity analysis, based on the two-way wave equation. It can be seen as a wave-equation analogue of ray-based reflection tomography (as described by Bishop et al., 1985, for example). The main difference with the above mentioned migration-based approach - which may also be seen as wave-equation reflection tomography - is that the misfit is measured in the data, rather than the image domain. This way, it is more closely linked to actual waveform inversion. However, we propose to use a different misfit criterion, which is based on a correlation of the modeled and observed data. We have employed such a misfit criterion earlier for NMO velocity analysis (van Leeuwen \& Mulder, 2008a) and transmission tomography (van Leeuwen \& Mulder, 2010b).

As classical reflection tomography requires the determination of reflector positions as part of the process, a natural framework to pose a wave-equation analogue is the Born approximation. We now have two unknowns: the reflectivity and the velocity. Least-squares estimation of the reflectivity then leads to a projection operator which projects the data onto the range of the modelling operator. The data and their projection will have different non-zero offset traveltimes when the velocity is not kinematically correct. Minimizing the least-squares difference between the data and their projection leads to a procedure known as migration-based traveltime tomography (MBTT) (Clément et al., 2001). Alternatively, the correlation-based misfit criteria may then be applied to measure the difference in traveltimes. 
The chapter is organized as follows. First, we briefly introduce the modelling framework and some notation. Then, we describe the projection operator and explain how it can be used to formulate a wave-equation reflection tomography problem. We discuss the correlation-based misfit criterion and derive the corresponding sensitivity kernel that can be used to update the velocity model. Finally, we present some examples on synthetic data.

\subsection{Modelling}

We assume that the wavefield, $u$, obeys a scalar wave equation of the form:

$$
\left[c(\mathbf{x})^{-2} \partial_{t}^{2}-\nabla^{2}\right] u\left(t, \mathbf{x}, \mathbf{x}_{s}\right)=f(t) \delta\left(\mathbf{x}-\mathbf{x}_{s}\right),
$$

where $c$ is the velocity, $f$ the source signature, and $\mathbf{x}_{s}$ is the source position. The data are a sampling of the wavefield at receiver locations $\mathbf{x}_{r}: d=\mathcal{S} u$. We introduce the modelling operator $\mathcal{F}$ such that

$$
d\left(t, \mathbf{x}_{s}, \mathbf{x}_{r}\right)=\mathcal{F}[c]
$$

We linearize around a smooth, reflection-free background velocity $c_{0}: c=c_{0}+$ $\delta c$. The total wavefield then consists of an incident wavefield $u_{0}$ and a scattered wavefield $\delta u$. Away from caustics the corresponding linearized, or Born, modelling operator is given asymptotically by

$$
\begin{aligned}
& \delta \mathcal{F}\left[c_{0}\right] \delta c \simeq-\frac{1}{\pi} \int \mathrm{d} \omega \int \mathrm{d} \mathbf{x} \omega^{2} \hat{f}(\omega) \frac{\delta c(\mathbf{x})}{c_{0}^{3}} \\
& \times A\left(\mathbf{x}_{s}, \mathbf{x}\right) A\left(\mathbf{x}, \mathbf{x}_{r}\right) \exp \left[\imath \omega\left(t-T\left(\mathbf{x}_{s}, \mathbf{x}\right)-T\left(\mathbf{x}, \mathbf{x}_{r}\right)\right)\right]
\end{aligned}
$$

where the traveltime obeys the eikonal equation

$$
\nabla T \cdot \nabla T=c_{0}^{-2}
$$

and $A$ obeys a transport equation. A derivation is given in the appendix. We will use this expression later on to analyze some of the kinematic aspects of the method.

\subsection{Separable least-squares}

We assume a known source signature and consider the problem of retrieving the velocity, $c$, from the measure data $\bar{d}$. The inverse problem can be cast as a PDEconstrained optimisation problem:

$$
c:=\underset{c}{\operatorname{argmin}}\|\mathcal{F}[c]-\bar{d}\|_{2}^{2} .
$$

This approach to seismic velocity inversion is called waveform inversion and was first considered by Tarantola \& Valette (1982).

To simplify the problem we consider the case where the data are well approximated 
by the linearization, introduced earlier. The partly linearized least-squares (LS) problem then reads

$$
\left\{c_{0}, \delta c\right\}:=\underset{c_{0}, \delta c}{\operatorname{argmin}}\left\|\delta \mathcal{F}\left[c_{0}\right] \delta c-\bar{d}\right\|_{2}^{2}
$$

Such a least-squares problem is called separable, since the unknowns separate into a linear and non-linear part. We consider applying the variable-projection approach (Golub \& Pereyra, 1973) to solve it. The idea is to eliminate $\delta c$ via the so-called normal equations:

$$
\delta c\left[c_{0}\right]=\mathcal{N}^{\dagger}\left[c_{0}\right] \delta \mathcal{F}^{*}\left[c_{0}\right] \bar{d},
$$

where $\mathcal{N}=\delta \mathcal{F}^{*} \delta \mathcal{F}$ is the normal operator or Hessian. Upon substituting this into equation (6.6) we get a modified least-squares optimisation problem:

$$
c_{0}:=\underset{c_{0}}{\operatorname{argmin}}\left\|\left(\pi\left[c_{0}\right]-I\right) \bar{d}\right\|_{2}^{2},
$$

where the projection operator, $\pi$, which projects the data onto the range of $\delta \mathcal{F}$ is given by

$$
\pi=\delta \mathcal{F} \mathcal{N}^{\dagger} \delta \mathcal{F}^{*}
$$

The range of the modelling operator changes rapidly as the background velocity is perturbed. In fact, the data and their projection for a perturbed model are nearly orthogonal in the high-frequency limit (Santosa \& Symes, 1989). This causes very large misfits unless the velocity perturbation is very small, making this approach not optimal for use with gradient-based optimisation methods, as has also been noted by Stolk \& Symes (2003). It should be mentioned however, that in the finitefrequency regime things may not be as bad. Clément et al. (2001) uses the variable projection approach to successfully overcome some of the shortcomings of classical waveform inversion.

Next, we will use the projection operator to formulate a wave-equation reflection tomography problem.

\subsection{Reflection tomography}

Traditionally, reflection tomography relies on raytracing. The traveltime of an event is picked in the data and the corresponding reflector position is estimated. Then, traveltimes are calculated by tracing the incoming and reflected rays. The traveltime mismatch between the picked and predicted events is used to update the velocity along the ray paths (Bishop et al., 1985). An extension of this method, called stereo tomography, does not only try to match the traveltimes but also the local slopes of events in the data (Billette \& Lambaré, 1998).

In this section we use the projection operator to formulate a wave-equation reflection tomography method. We note that the reflection tomography can also be formulated in terms of forward propagated source wavefields and backwards propagated receiver wavefields. This leads to various instances of migration velocity analysis (Doherty \& Claerbout, 1974; Symes \& Carazzone, 1991; Sava \& Biondi, 2004; de Hoop \& van der Hilst, 2005; Shen \& Symes, 2008). 


\subsubsection{Projection}

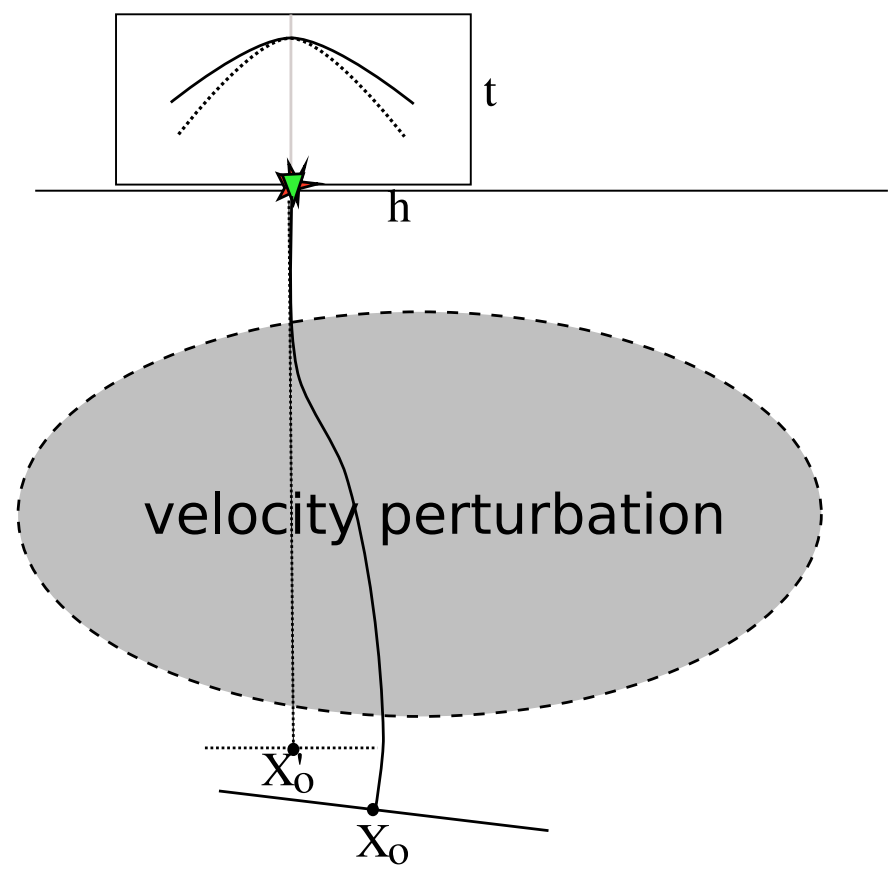

Figure 6.1. Kinematics of the projection operator. The zero offset ray between the reflector segment at $\mathbf{x}_{0}$ and the surface in the true model is depicted with a solid line. In absence of the velocity perturbation, the data is imaged by simply tracing the ray back to half the traveltime (dashed line), resulting in a reflector segment at $\mathbf{x}_{0}^{\prime}$. While this retains the zero-offset traveltime, the wavefront curvature will be different.

To investigate the kinematics of the projection operator, we consider models with mild lateral variation. For simplicity we consider an experiment along a line for a fixed midpoint: $x_{m}=\left(x_{r}+x_{s}\right) / 2$. We also introduce the source-receiver offset $h=\left(x_{r}-x_{s}\right) / 2$. The two-way traveltime to a specific reflection point $\mathbf{x}_{0}$ can now be approximated by

$$
T\left(\mathbf{x}_{0}, x_{s}\right)+T\left(\mathbf{x}_{0}, x_{r}\right) \approx 2 T\left(\mathbf{x}_{0}, x_{m}\right)+h^{2} \Gamma\left(\mathbf{x}_{0}, x_{m}\right),
$$

where $\Gamma=\frac{\partial^{2} T}{\partial h^{2}}$ is related to the wavefront curvature.

The projection onto the range of the modelling operator insures that the zerooffset traveltimes are retained. The kinematic difference between the data and their projection lies in the traveltimes at non-zero offsets. The situation is depicted schematically in figure 6.1. Examples are given in section 6.6. Next, we discuss a way to measure the traveltime shift. 


\subsubsection{Correlation: detection of traveltime shifts}

The use of temporal correlation to detect the shift between 1D wavefields is wellestablished (Cara \& Leveque, 1987; Luo \& Schuster, 1991; Gee \& Jordan, 1992; Hörmann \& de Hoop, 2002; de Hoop \& van der Hilst, 2005; van Leeuwen \& Mulder, 2010b) and can be applied in this case for each offset-trace separately.

Following van Leeuwen \& Mulder (2008a), we consider the temporal and spatial correlation of a fixed midpoint gather $d(t, h):=d\left(t, x_{m}-h, x_{m}+h\right)$ :

$$
\begin{aligned}
C_{t}[d, \bar{d}](\Delta t, h) & =\int \mathrm{d} t \bar{d}(t+\Delta t, h) d(t, h), \\
C_{h}[d, \bar{d}](t, \Delta h) & =\int \mathrm{d} h \bar{d}(t, h+\Delta h) d(t, h) .
\end{aligned}
$$

The correlation will reveal the difference in moveout between the midpoint gathers by a concentration of energy at non-zero shift. Some examples are given in section 6.6. A detailed analysis of the kinematics of such correlation functions is given by van Leeuwen \& Mulder (2010a) (reproduced in chapter 4).

To measure the misfit, we use a weighted norm of the correlation

$$
J\left[c_{0}\right]=\|W \cdot C[d, \bar{d}]\|_{2}^{2}, \quad d=\pi\left[c_{0}\right] \bar{d},
$$

where the weight penalizes energy away from zero shift, in which case the functional is to be minimized, or measures the energy around zero shift, in which case the functional is to be maximized. More details on measuring the traveltime shift via correlation are given by van Leeuwen \& Mulder (2008a, 2010a,b) (reproduced in chapters 3-5) and references cited therein.

\subsection{Sensitivity Kernel}

To update the velocity via a gradient-based optimisation method we need the Fréchet derivative of the misfit functional w.r.t. to the velocity. This gradient, sometimes called the sensitivity kernel, relates velocity perturbations to changes in the misfit via:

$$
J\left[c_{0}+\Delta c_{0}\right]-J\left[c_{0}\right] \approx \int \mathrm{d} \mathbf{x} K(\mathbf{x}) \Delta c(\mathbf{x})
$$

Formally, the sensitivity kernel is given by

$$
\begin{aligned}
K(\mathbf{x}) & =\partial_{c_{0}} J=\left[\partial_{c_{0}}(\pi \bar{d})\right]^{*} e[d, \bar{d}] \\
& =(\underbrace{\delta c\left(\partial_{c_{0}} \delta \mathcal{F}[c]\right)^{*}}_{(\mathrm{A})}+\underbrace{\left(\partial_{c_{0}} \delta c\right) \delta \mathcal{F}^{*}[c]}_{(\mathrm{B})}) e[d, \bar{d}]
\end{aligned}
$$

where the adjoint source, $e$ is the Fréchet derivative of the misfit functional w.r.t. the data, $\delta c=\mathcal{N}^{\dagger} \delta \mathcal{F}^{*} \bar{d}$ and $d=\pi \bar{d}$. For the modified least-squares approach, for 
example, $e[d, \bar{d}]=2(d-\bar{d})$. In this case it is readily verified that the second term (B) vanishes. For the correlation-based misfits, however, both terms are expected to contribute significantly.

In the appendix we give a detailed derivation of how to calculate the kernel in terms of wavefields. We may subsequently use the asymptotic expression for the Green function to gain some more insight in the structure of the kernel. For simplicity we will consider only the first term (A), ignoring the sensitivity of $\delta c$ w.r.t. $c_{0}$. As derived in the appendix, this yields:

$$
K(\mathbf{x})=\sum_{i=0}^{1} \int \mathrm{d} \mathbf{x}_{s} \int \mathrm{d} \mathbf{x}_{r} \int \mathrm{d} \omega \omega^{2} f(\omega) K^{(i)}\left(\omega, \mathbf{x}_{s}, \mathbf{x}, \mathbf{x}_{r}\right) \hat{e}\left(\omega, \mathbf{x}_{s}, \mathbf{x}_{r}\right),
$$

where $\hat{e}$ the temporal Fourier transform of $e$ and

$$
\begin{aligned}
& K^{(0)}\left(\omega, \mathbf{x}_{s}, \mathbf{x}, \mathbf{x}_{r}\right)= \\
& \quad 2 \int \mathrm{d} \mathbf{x}^{\prime} A\left(\mathbf{x}_{s}, \mathbf{x}, \mathbf{x}^{\prime}, \mathbf{x}_{r}\right) \frac{\delta c\left(\mathbf{x}^{\prime}\right)}{c_{0}\left(\mathbf{x}^{\prime}\right)} \exp \left[\imath \omega\left(T\left(\mathbf{x}_{s}, \mathbf{x}^{\prime}\right)+T\left(\mathbf{x}^{\prime}, \mathbf{x}\right)+T\left(\mathbf{x}, \mathbf{x}_{r}\right)\right)\right], \\
& K^{(1)}\left(\omega, \mathbf{x}_{s}, \mathbf{x}, \mathbf{x}_{r}\right)= \\
& \quad 2 \int \mathrm{d} \mathbf{x}^{\prime} A\left(\mathbf{x}_{s}, \mathbf{x}, \mathbf{x}^{\prime}, \mathbf{x}_{r}\right) \frac{\delta c\left(\mathbf{x}^{\prime}\right)}{c_{0}\left(\mathbf{x}^{\prime}\right)} \exp \left[\imath \omega\left(T\left(\mathbf{x}_{s}, \mathbf{x}\right)+T\left(\mathbf{x}, \mathbf{x}^{\prime}\right)+T\left(\mathbf{x}^{\prime}, \mathbf{x}_{r}\right)\right)\right] .
\end{aligned}
$$

When we consider a single source-receiver pair and a single reflection point $\mathbf{x}_{0}$ (i.e., $\delta c(\mathbf{x})=\delta\left(\mathbf{x}-\mathbf{x}_{0}\right)$ ), the difference between the two terms described above becomes clear. $K^{(1)}(\mathbf{x})$ describes a ray: $\mathbf{x}_{s}-\mathbf{x}_{0}-\mathbf{x}^{\prime}-\mathbf{x}_{r}$ while $K^{(2)}(\mathbf{x})$ describes a ray: $\mathbf{x}_{s}-\mathbf{x}-\mathbf{x}_{0}-\mathbf{x}_{r}$. This geometry yields the characteristic double banana-shape due to constructive interference of the wavefields within the Fresnel-zone around the central ray (de Hoop et al., 2006). The situation is depicted in figure 6.2 and examples are given in the next section.

\subsection{Examples}

The above described approach would require solving the normal equations for each velocity model, which is not feasible in practice. Instead, we exploit the pseudodifferential nature of the Hessian (Beylkin, 1984):

$$
\mathcal{N} \simeq \alpha(\mathbf{x}) \Delta^{(n-1) / 2},
$$

where $n$ is the spatial dimension. The scaling factor $\alpha$ may be determined by optimally matching $r_{0}=\delta \mathcal{F}^{*} \bar{d}$ with $r_{1}=\delta \mathcal{F}^{*} \delta \mathcal{F} r_{0}$ (Symes, 2008a). However, for simplicity we choose a fixed scalar factor $\alpha$. The projected data are then computed as follows:

1. Migrate the observed data: $r=\delta \mathcal{F}^{*}\left[c_{0}\right] \bar{d}$

2. Filter and scale the reflectivity estimate: $\delta c=\mathcal{N}^{\dagger} r$

3. Model data for the filtered reflectivity estimate: $d=\delta \mathcal{F}\left[c_{0}\right] \delta c$ 


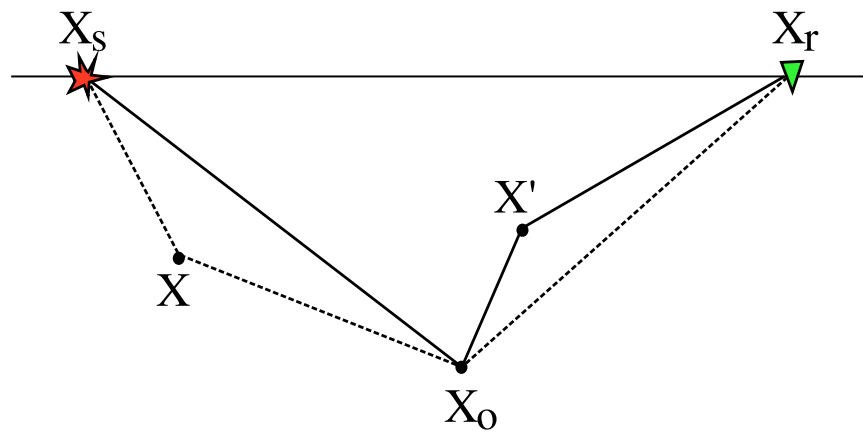

Figure 6.2. Schematic depiction of the reflection tomography sensitivity kernel for one source-receiver pair and one reflection point. The solid and dashed line depict the two different terms from equation (6.16). In the high-frequency limit, the update is confined to the 'central' ray $\mathbf{x}_{s}-\mathbf{x}_{0}-\mathbf{x}_{r}$. In the finite frequency regime, constructive interaction of the wavefields away from the central ray lead to the double banana shape.

In the appendix we give a detailed description of how to calculate the projection in terms of wavefields.

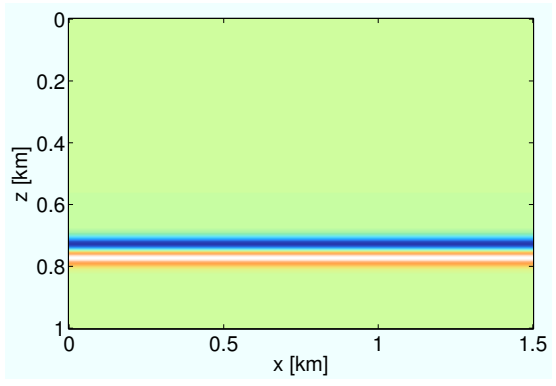

(a)

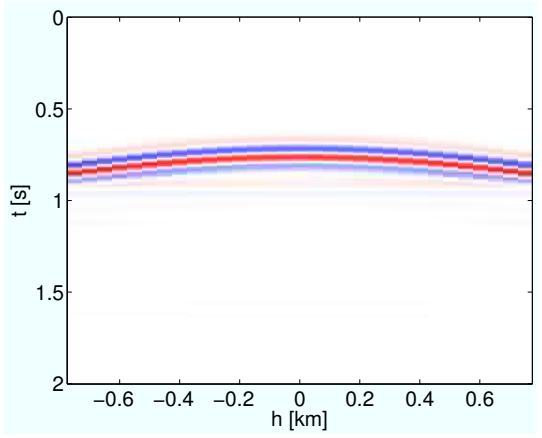

(b)

Figure 6.3. (a) Reflector in constant velocity of $2 \mathrm{~km} / \mathrm{s}$ and (b) corresponding midpoint gather at $x_{m}=750 \mathrm{~m}$. As a source wavelet we used a Ricker wavelet with a $10 \mathrm{~Hz}$ peak frequency.

\subsubsection{Scan}

We consider a constant velocity with one reflector, see figure 6.3. The projections (migration-demigration) of these data for different constant velocities are depicted in figure 6.4. The corresponding temporal and spatial correlation panels (cf. equation (6.12)) are depicted in figure 6.5. For the correct velocity figure 6.5(b,e) the 
energy should be focused at zero shift. Note the non-focusing artefact in the spatial correlation. We refer to van Leeuwen \& Mulder (2010a) (chapter 4) for a detailed analysis of the kinematics of such correlation panels.

The functional is then evaluated for different constant velocities and the result

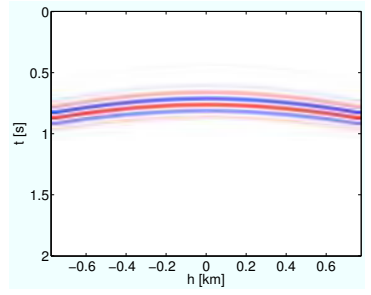

(a)

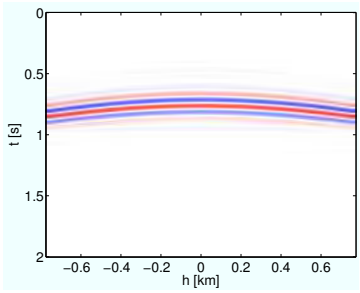

(b)

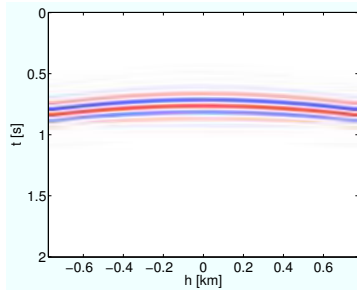

(c)

Figure 6.4. Projected data for a constant velocity of (a) $1.8 \mathrm{~km} / \mathrm{s}$, (b) $2 \mathrm{~km} / \mathrm{s}$, and (c) $2.2 \mathrm{~km} / \mathrm{s}$.

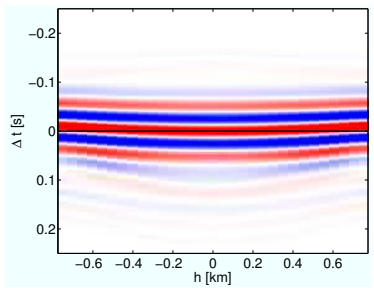

(a)

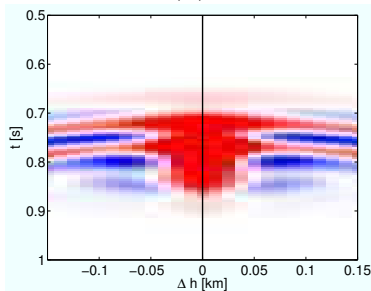

(d)

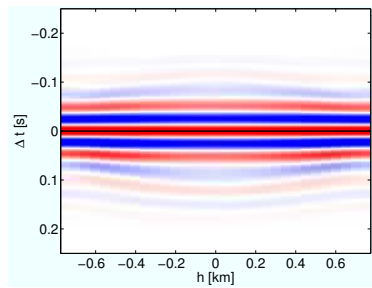

(b)

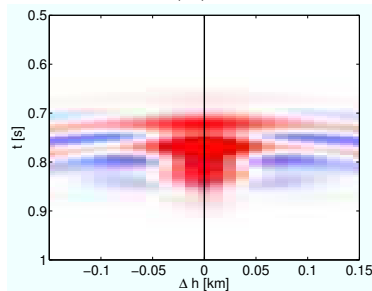

(e)

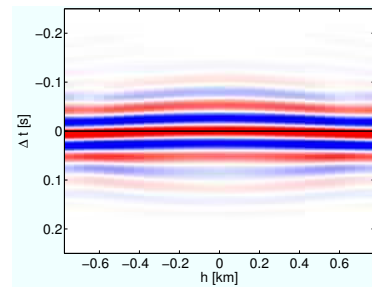

(c)

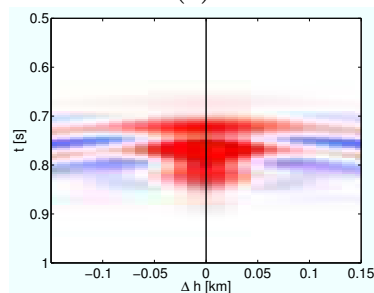

(f)

Figure 6.5. Temporal $(a-c)$ and spatial $(d-f)$ correlation of the project data from figure 6.4 with the observed data from figure 6.3. For the correct velocity $(b, e)$ the energy should be focused around zero shift. The focussing in the spatial correlation is not optimally visible due to the relatively low peak frequency and the short offset. Note the non-focusing artefact in the spatial correlation.

is shown in figure 6.6. The weighting function is a Gaussian, as described in the previous chapters. 


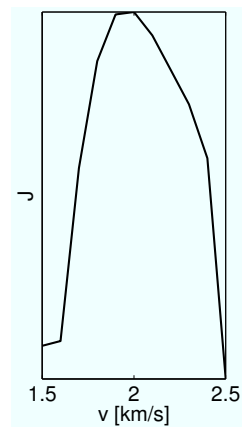

(a)

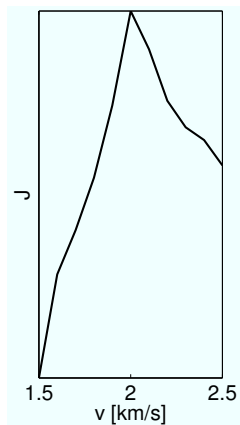

(b)

Figure 6.6. Correlation-based misfit functional as a function of the background velocity. (a) temporal correlation and (b) spatial correlation.

\subsubsection{Gradient}

We calculate the gradient according to equation (6.16). The appendix gives a detailed derivation of how to compute the gradient with the adjoint-state technique. Figure 6.7 gives an example of the first term of the gradient (A) for one sourcereceiver pair and one reflection point. This illustrates the characteristic shape of the sensitivity kernel, as explained above. Figure 6.8 displays the gradient as it would be used to update the velocity. There is a strong acquisition and reflector imprint. To avoid these kind of oscillatory artefacts in the velocity update the gradient is usually smoothed or projected on splines.

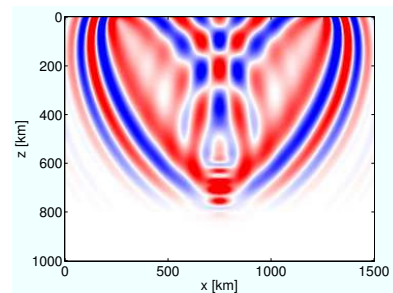

(a)

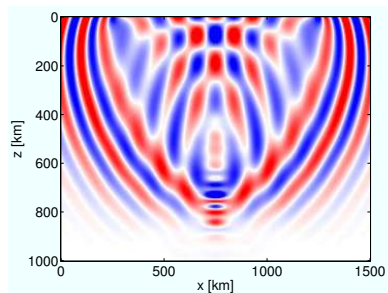

(b)

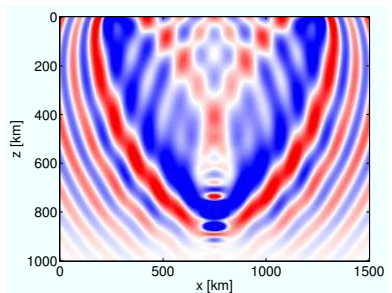

(c)

Figure 6.7. Sensitivity kernels corresponding to the correlation panels from figure 6.4 for a single source receiver pair (250,1250) $m$ and one reflection point. The velocity is (a) too low, (b) exact, and (c) too high. Note that the sign of the kernels changes accordingly.

\subsubsection{Inversion}

To test the proposed method we use the model in figure 6.9(a). We solve the wave equation with a frequency-domain finite difference code. The resulting data are depicted in figure 6.9(b). We use the temporal correlation and a Gaussian weight 

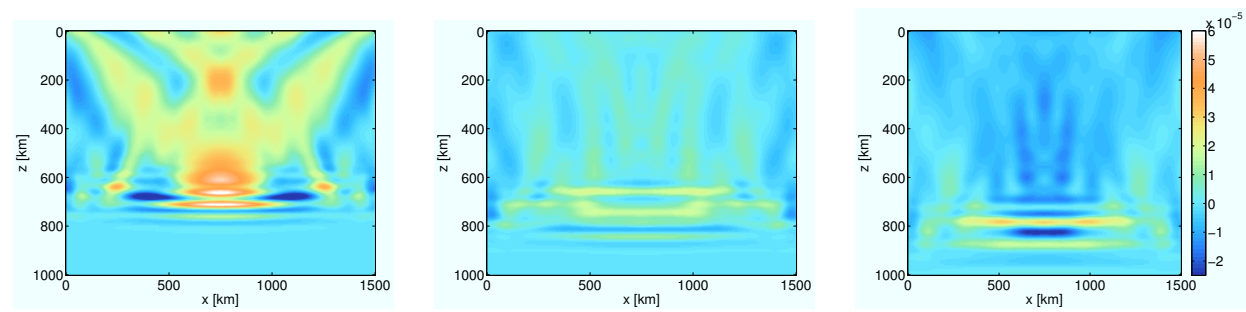

Figure 6.8. Gradient corresponding to the correlation panels from figure 6.4. The velocity is (a) too low, (b) exact, and (c) too high. Note that the sign of the gradient changes accordingly. Although the overall sign of the gradient is correct, there is a strong acquisition and reflector imprint. For practical applications the gradient needs to be projected on splines to prevent unwanted oscillatory artefacts in the velocity update.

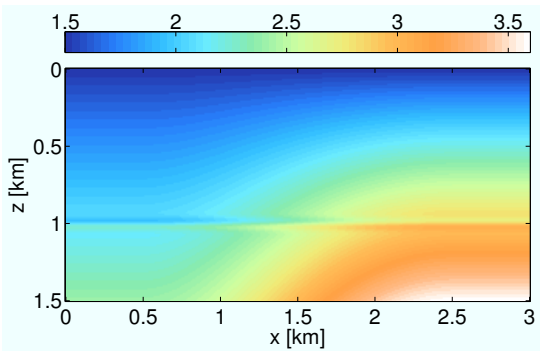

(a)

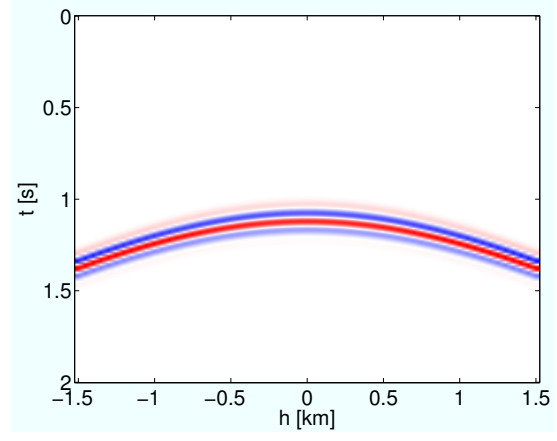

(b)

Figure 6.9. Velocity model (a) and midpoint gather of corresponding data (b) used for test.

to measure the misfit. To remove low-frequency artefacts in the migrated image, we apply a high-pass filter. To avoid high-frequency artefacts in the velocity update, we project the gradient on linear splines. The results of two experiments are depicted in figure 6.10 and figure 6.11. Although we did not retrieve the velocity perfectly, the figures show that the correlation panels are nicely focused, indicating that the events are close enough to allow for least-squares fitting of the data.

\subsection{Conclusion \& discussion}

We formulated an approach for wave-equation reflection tomography for singly scattered data. This approach relies on a projection (migration-demigration) of the data and a subsequent evaluation of a correlation-based misfit criterion for each trial velocity. We illustrated the kinematics of the projection procedure on a simple synthetic, one-reflector example. We also derive the corresponding sensitivity 


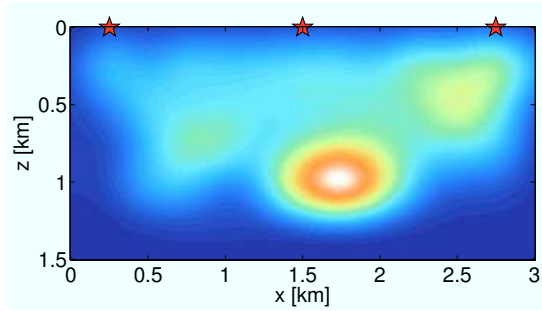

(a)

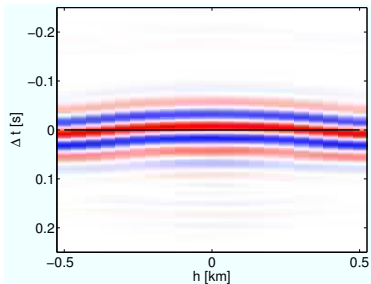

(c)

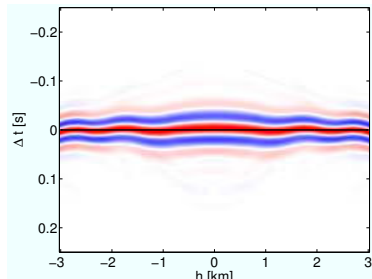

(d)

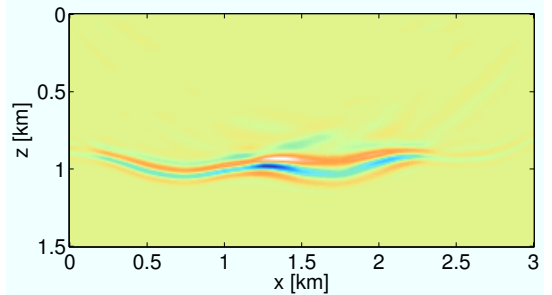

(b)

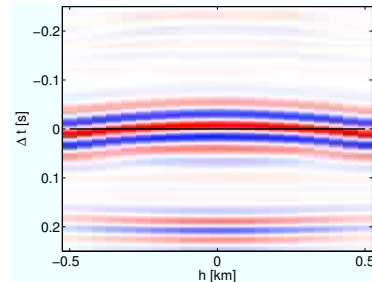

(e)

Figure 6.10. As initial model we used a constant velocity of $1500 \mathrm{~m} / \mathrm{s}$. We used a spline grid with a spacing of $250 \mathrm{~m}$. The final velocity and reflectivity after 21 iterations are depicted in $(a, b)$. The corresponding correlation panels for the midpoint positions indicated in (a) are depicted in $(c-f)$.

kernel. The gradient of the misfit shows a strong acquisition and reflector imprint, which necessitates extra regularisation of the gradient to avoid oscillatory artefacts in the velocity update.

Finally, we illustrate the feasibility of the approach with an inversion of synthetic data for a laterally varying one-reflector model. The examples show that without sufficient regularisation of the updates, there might be too many degrees of freedom and the optimisation can converge to an undesirable solution. In particular, the illumination and amplitudes of the events change with the velocity. This way, events that would cause a defocusing in the correlation panel may be eliminated by shifting them out of the acquisition. We chose to use a spline grid that has a larger spacing to regularise the updates. It remains to be seen if this is sufficient in case of strong lateral variation and multiple reflectors. It is clear that multiple reflectors would help constrain the velocity better. On the other hand, we expect difficulties in weighting the different events in the data properly. However, we expect that the same kind of challenges when devising any other wave-equation based velocity updating scheme such wave-equation migration velocity analysis. Getting this right will remain one of the main challenges of seismic imaging in the near future. 


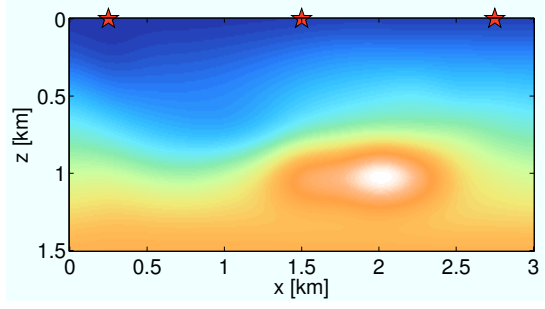

(a)

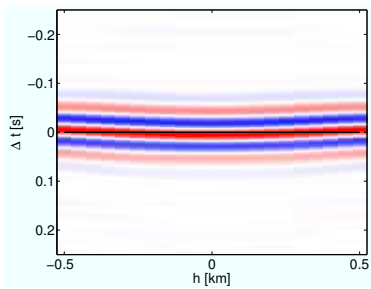

(c)

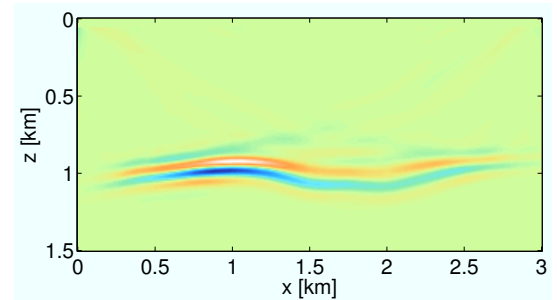

(b)

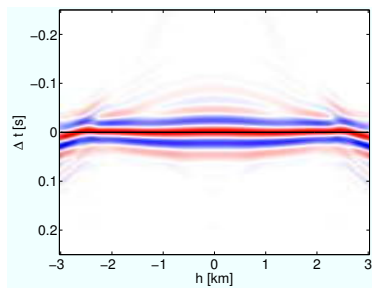

(d)

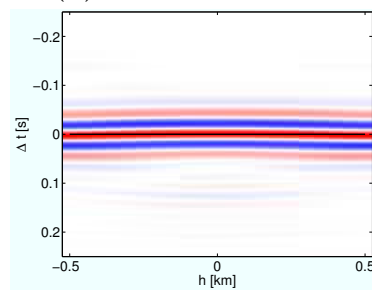

(e)

Figure 6.11. As initial model we used the optimal linear velocity: $1500+\alpha z$, where $\alpha$ was found by a line search. We used a spline grid with a spacing of $250 \mathrm{~m}$. The final velocity and reflectivity after 15 iterations are depicted in $(a, b)$. The corresponding correlation panels for the midpoint positions indicated in (a) are depicted in $(c-f)$. 


\subsection{Appendix}

We use the following definition of the Fourier transform

$$
\begin{aligned}
\hat{g}(\omega) & =\int \mathrm{d} t g(t) \exp [-\imath \omega t], \\
g(t) & =\frac{1}{2 \pi} \int \mathrm{d} \omega \hat{g}(\omega) \exp [\imath \omega t] .
\end{aligned}
$$

The wave equation reads

$$
\underbrace{\left[c(\mathbf{x})^{-2} \partial_{t}^{2}-\nabla^{2}\right]}_{\mathcal{L}[c]} u\left(t, \mathbf{x}, \mathbf{x}_{s}\right)=f(t) \delta\left(\mathbf{x}-\mathbf{x}_{s}\right) .
$$

We will drop the dependence of the wavefields on $\left(t, \mathbf{x}, \mathbf{x}_{s}\right)$ and absorb the source position $\delta\left(\mathbf{x}-\mathbf{x}_{s}\right)$ into $f$ when no confusion is possible in the remainder of the appendix.

\subsection{1 $\delta \mathcal{F}\left[c_{0}\right]$}

Upon splitting the velocity in a smooth and rough component, $c:=c_{0}+\delta c$, and the wavefield in an incident and scattered field, $u=u_{0}+\delta u$, and applying the Born approximation we find:

$$
\begin{aligned}
& \mathcal{L}\left[c_{0}\right] u_{0}=f, \\
& \mathcal{L}\left[c_{0}\right] u_{1}=2 u_{0}^{\prime \prime} c_{0}^{-3} \delta c,
\end{aligned}
$$

where' denotes the temporal derivative. In the high-frequency limit, away from caustics, the Green function for the background velocity $c_{0}$ is given by

$$
G_{0}\left(t, \mathbf{x}, \mathbf{x}^{\prime}\right) \simeq A\left(\mathbf{x}, \mathbf{x}^{\prime}\right) \delta\left[t-T\left(\mathbf{x}, \mathbf{x}^{\prime}\right)\right],
$$

where $T$ satisfies the eikonal equation and $A$ satisfies a transport equation. The scattered field is then approximated by

$$
\begin{aligned}
u_{1}\left(t, \mathbf{x}_{s}, \mathbf{x}\right)=-\frac{1}{\pi} & \int \mathrm{d} \omega \int \mathrm{d} \mathbf{x}^{\prime} \omega^{2} \hat{f}(\omega) \frac{\delta c\left(\mathbf{x}^{\prime}\right)}{c_{0}\left(\mathbf{x}^{\prime}\right)^{-3}} A\left(\mathbf{x}_{s}, \mathbf{x}^{\prime}\right) A\left(\mathbf{x}^{\prime}, \mathbf{x}\right) \\
& \times \exp \left[\imath \omega\left(t-T\left(\mathbf{x}_{s}, \mathbf{x}^{\prime}\right)-T\left(\mathbf{x}^{\prime}, \mathbf{x}\right)\right)\right] .
\end{aligned}
$$

Assuming that the data are a sampling of the scattered field only then leads to equation (6.3).

\subsection{2 $\delta \mathcal{F}^{*}\left[c_{0}\right]$}

We introduce the least-squares functional:

$$
J_{\mathrm{LS}}=\left\|\delta \mathcal{F}\left[c_{0}\right] \delta c-\bar{d}\right\|_{2}^{2} .
$$


The formal derivative of the LS functional w.r.t $\delta c$ is then given by

$$
\partial_{\delta c} J_{\mathrm{LS}}(\mathbf{x})=2 \delta \mathcal{F}^{*}\left(\delta \mathcal{F}\left[c_{0}\right] \delta c-\bar{d}\right) .
$$

Hence, the action of the adjoint is given by

$$
\delta \mathcal{F}^{*} \bar{d}=-\frac{1}{2} \partial_{\delta c} J_{\mathrm{LS}}\left[c_{0}, \delta c=0\right] .
$$

The Fréchet derivative of the LS functional is readily calculated via the adjoint-state technique. We introduce the Lagrangian:

$$
L\left[u_{1}, \delta c\right]=J_{\mathrm{LS}}+\left\langle\lambda_{0}, \mathcal{L}\left[c_{0}\right] u_{1}-2 u_{0}^{\prime \prime} c_{0}^{-3} \delta c\right\rangle,
$$

where we have substituted $\delta \mathcal{F}\left[c_{0}\right] \delta c=\mathcal{S} u_{1}$ in the LS functional. The derivatives of the Lagrangian are given by

$$
\begin{aligned}
\partial_{u_{1}} L & =2 \mathcal{S}^{*}\left(\mathcal{S} u_{1}-\bar{d}\right)+\mathcal{L}^{*}\left[c_{0}\right] \lambda_{0}, \\
\partial_{\delta c} L & =-2 c_{0}^{-3}\left\langle u_{0}^{\prime \prime}, \lambda_{0}\right\rangle .
\end{aligned}
$$

Then, $\partial_{\delta c} J_{\mathrm{LS}}=\partial_{\delta c} L$ subject to $\partial_{u_{1}} L=0$. We find:

$$
\partial_{\delta c} J_{L S}=-c_{0}^{-3}\left\langle u_{0}^{\prime \prime}, v_{0}\right\rangle, \mathcal{L}^{*}\left[c_{0}\right] v_{0}=-\mathcal{S}^{*}\left(\mathcal{S} u_{1}-\bar{d}\right) .
$$

Hence,

$$
\delta \mathcal{F}^{*}\left[c_{0}\right] \bar{d}=\frac{1}{2} c_{0}^{-3}\left\langle u_{0}^{\prime \prime}, v_{0}\right\rangle, \mathcal{L}\left[c_{0}\right] u_{0}=f, \mathcal{L}^{*}\left[c_{0}\right] v_{0}=\mathcal{S}^{*} \bar{d}
$$

\subsection{3 $\partial_{c}\left(\pi\left[c_{0}\right] \bar{d}\right)$}

We now consider a functional that measures the misfit between the data, $\bar{d}$, and their projection $d=\pi\left[c_{0}\right] \bar{d}$. We will first elaborate on the procedure to calculate the projection and subsequently derive the Fréchet derivative of the functional w.r.t the velocity.

The projection can be easily calculated in three steps:

1. perform a reverse time migration of the data to obtain an image $r$,

2. filter and scale the output of the RTM appropriately to obtain an estimate of the reflectivity $\delta c=\mathcal{N}^{\dagger} r$,

3. model data for the estimated reflectivity (demigration).

In terms of wavefields, the projected data are then given by $d=\mathcal{S} \tilde{u}_{1}$, where

$$
\begin{aligned}
\mathcal{L}\left[c_{0}\right] u_{0} & =f, \\
\mathcal{L}^{*}\left[c_{0}\right] v_{0} & =\mathcal{S}^{*} \bar{d}, \\
\delta c & =\frac{1}{2} c_{0}^{-3} \mathcal{N}^{\dagger}\left\langle u_{0}^{\prime \prime}, v_{0}\right\rangle, \\
\mathcal{L}\left[c_{0}\right] \tilde{u}_{0} & =f, \\
\mathcal{L}\left[c_{0}\right] \tilde{u}_{1} & =2 c_{0}^{-3} \tilde{u}_{0}^{\prime \prime} \delta c .
\end{aligned}
$$


The corresponding Lagrangian is given by

$$
\begin{aligned}
& L\left[u_{0}, v_{0}, \tilde{u}_{0}, \tilde{u}_{1}, c, \delta c\right]=J\left[\mathcal{S} \tilde{u}_{1}, \bar{d}\right] \\
& +\left\langle\lambda_{0}, \mathcal{L}\left[c_{0}\right] \tilde{u}_{0}-f\right\rangle+\left\langle\lambda_{1}, \mathcal{L}\left[c_{0}\right] \tilde{u}_{1}-2 c_{0}^{-3} \tilde{u}_{0}^{\prime \prime} \delta c\right\rangle \\
& +\left\langle\lambda_{2}, \delta c-\frac{1}{2} c_{0}^{-3} \mathcal{N}^{\dagger}\left\langle u_{0}^{\prime \prime}, v_{0}\right\rangle\right\rangle+\left\langle\lambda_{3}, \mathcal{L}\left[c_{0}\right] u_{0}-f\right\rangle+\left\langle\lambda_{4}, \mathcal{L}^{*}\left[c_{0}\right] v_{0}-\mathcal{S}^{*} \bar{d}\right\rangle
\end{aligned}
$$

The Fréchet derivatives of the Lagrangian are given by

$$
\begin{aligned}
\partial_{u_{0}} L & =\mathcal{L}^{*}\left[c_{0}\right] \lambda_{3}-\frac{1}{2} c_{0}^{-3} v_{0}^{\prime \prime} \mathcal{N}^{\dagger} \lambda_{2}, \\
\partial_{v_{0}} L & =\mathcal{L}\left[c_{0}\right] \lambda_{4}-\frac{1}{2} c_{0}^{-3} u_{0}^{\prime \prime} \mathcal{N}^{\dagger} \lambda_{2}, \\
\partial_{\delta c} L & =\lambda_{2}-2 c_{0}^{-3}\left\langle\tilde{u}_{0}^{\prime \prime}, \lambda_{1}\right\rangle, \\
\partial_{\tilde{u}_{0}} L & =\mathcal{L}^{*} \lambda_{0}-2 c_{0}^{-3} \lambda_{1}^{\prime \prime} \delta c \\
\partial_{\tilde{u}_{1}} L & =\mathcal{L}^{*} \lambda_{1}+\mathcal{S}^{*} \partial_{d} J \\
\partial_{c_{0}} L & =\left\langle\lambda_{0}, \tilde{u}_{0}^{\prime \prime}\right\rangle+\left\langle\lambda_{1}, \tilde{u}_{1}^{\prime \prime}\right\rangle+\left\langle\lambda_{3}, u_{0}^{\prime \prime}\right\rangle+\left\langle\lambda_{4}^{\prime \prime}, v_{0}\right\rangle .
\end{aligned}
$$

With $\lambda_{0}=\tilde{v}_{1}, \lambda_{1}=\tilde{v}_{0}, \widetilde{\delta c}=\frac{1}{4} \mathcal{N}^{\dagger} \lambda_{2}, \lambda_{3}=\tilde{v}_{1}, \lambda_{4}=u_{1}$ This yields

$$
\begin{aligned}
\partial_{c_{0}} J & =\underbrace{\left\langle\tilde{u}_{0}^{\prime \prime}, \tilde{v}_{1}\right\rangle+\left\langle\tilde{u}_{1}^{\prime \prime}, \tilde{v}_{0}\right\rangle}_{(\mathrm{A})}+\underbrace{\left\langle u_{0}^{\prime \prime}, v_{1}\right\rangle+\left\langle u_{1}^{\prime \prime}, v_{0}\right\rangle}_{(\mathrm{B})}, \\
\mathcal{L}^{*} \tilde{v}_{0} & =-\mathcal{S}^{*} \partial_{d} J \\
\mathcal{L}^{*} \tilde{v}_{1} & =2 c_{0}^{-3} \tilde{v}_{0}^{\prime \prime} \delta c \\
\widetilde{\delta c} & =\frac{1}{2} c_{0}^{-3} \mathcal{N}^{\dagger}\left\langle\tilde{u}_{0}^{\prime \prime}, \tilde{v}_{0}\right\rangle, \\
\mathcal{L} u_{1} & =2 c_{0}^{-3} v_{0}^{\prime \prime} \tilde{\delta c} \\
\mathcal{L}^{*} v_{1} & =2 c_{0}^{-3} u_{0}^{\prime \prime} \tilde{\delta c} .
\end{aligned}
$$

Together with the asymptotic expression of Green's function equation (6.25), this leads to equation (6.16)-equation (6.18). 


\section{7 \\ Conclusions and outlook}


In this thesis we have described an approach to wave-equation (reflection) traveltime tomography in the context of linearized modelling. In contrast to classical traveltime tomography, which relies on picking traveltimes and raytracing, the methodology proposed here relies on full waveform modelling of the data to predict the relevant arrivals. Traveltime tomography in terms of full wavefields is not new in itself, however. Key aspects that make this work different are:

- The domain in which the misfit is measured. Existing methods for wave-equation reflection tomography measure a focusing of wavefields in depth and sub-surface offset. This requires an extended modelling concept. In this thesis, we propose to measure the misfit in the data domain. This requires an intermediate step to determine the reflectivity and this leads to the introduction of a projection operator that projects the data onto the range of the modelling operator.

- The way in which the misfit is measured. The weighted norm that we propose to use to measure the misfit based on the correlation borrows ideas from migration velocity analysis in the sense that it measures a focussing of wavefields. At the same time, its use in detection of time shifts in transmission tomography seems to be a novelty. In this context we may view the weighted norm approach as a finite-frequency generalization of the 'picking approach', as is described in chapter 5 (van Leeuwen \& Mulder, 2010b).

The ultimate goal would be to develop a fully automated method to obtain an acceptable velocity, even in the presence of multiple reflections. To this end we have to move beyond the linearized modelling framework. While the extended modelling concept can be generalized beyond linear modelling (Symes, 2008b) there are some practical issues that hamper its immediate application to life-size problems. We believe that the framework proposed here provides an interesting alternative avenue for further investigation of velocity estimation in the presence of multiples.

Currently, however, wave-equation-based velocity updating is far from being wellunderstood. Although application to transmission data - such as diving waves - is underway, the classical least-squares formalism that is being employed still relies on low frequencies. Application to reflection data - where one cannot rely on low frequencies - will remain one of the main challenges in seismic imaging for the nearby future.

Some immediate research opportunities regarding the method described in this thesis are:

- Extension of the projection operator to account for multiple reflections,

- Better understanding of how an approximate projection affects convergence etc.,

- Better understanding of the correlation-based misfit criterion,

- Optimization of computational efficiency,

- Traveltime tomography vs. waveform inversion.

In the following we briefly discuss these issues. 


\subsection{Extension of the projection operator}

To successfully include multiples the projection operator should be extended beyond the linearized setting. In particular, this means that the migration step should map multiples to their corresponding reflectors without producing ghost reflectors. To achieve this, we may pre-process the data to transform multiples into primaries or we can rely on a linearization around a non-smooth model, relying on a layerstripping approach to determined the reflectors from the top down. A proof of concept of the latter in the context of NMO modelling is given in (van Leeuwen \& Mulder, 2008c).

\subsection{Approximate projection}

The projection operator arises when applying the variable projection approach to solve a separable least-squares problem. It is interesting to investigate how convergence is affected by approximating the Hessian.

\subsection{Correlation-based misfit}

The correlation-based misfit seems well-suited to measure the phase shift between single arrivals. When correlating multiple arrivals at the same time, cross-talk between the different events arises. It is not quite clear to what extend the focussing principle still holds. Also, to handle full wavefields, the sensitivity to slowness differences needs to be investigated. This would allow for a wave-equation stereotomography approach. As correlation-based misfit actually measures differences in the wavefrontset of the wavefields it may be more natural to define the misfit in the curvelet domain. This would also allow for filtering of unwanted events. Finally, since the Gaussian-weighted norm approach includes a scale parameter, this would allow for a multi-scale mismatch measurement. In particular, we expect that this can be exploited to measure dispersion.

\subsection{Computational efficiency}

The projection operator is expensive to evaluate and reasonable approximations to the Hessian need to be incorporated. It seems that the kind of approximation used has a strong influence on the method. Also, much could be gained by figuring out how to successfully apply the method to update only a part of the velocity model. This would avoid working with the whole dataset. A recent development is the use of a multi-shot approach for wave-equation migration, where several shots are combined to save computation time.

\subsection{Tomography vs. waveform inversion}

In the high-frequency limit, for short-offset reflection data the phase of the data contain mostly information on the smooth velocity while the amplitudes contain mostly information on the reflectivity. The correlation-based criteria (tomography) 
seem to be optimal for inverting the phases of the data, while the classical leastsquares approach is optimal for inverting the amplitudes. When low frequencies or large offsets are available, the clear distinction between amplitude and phase sensitivity to different components of the model begins to fade. Is there an optimal way to deal with the amplitude and the phase properly at the same time? For the short-offset reflection case, the projection operator does exactly this; it allows us to 'cruise around in the null-space' seeking the optimal velocity while keeping the amplitude fit more or less the same. It is not quite clear how to move beyond the Born approximation here. 


\section{Bibliography}

Bednar, J. 2005. A brief history of seismic migration $[\hookrightarrow]$. Geophysics, 70(3), 3MJ20MJ. 4.1

Beylkin, G. 1984. The inversion problem and applications of the generalized radon transform $[\hookrightarrow]$. Communications on Pure and Applied Mathetmatics, 37, 579-599. $2.3 .2,2.3 .2,2.3 .2,6.6$

Beylkin, G. 1985. Imaging of discontinuities in the inverse scattering problem by inversion of a causal generalized radon transform $[\hookrightarrow]$. Journal of Mathematical Physics, 26(1), 99-108. 2.3.2, 4.4

Billette, F., \& Lambaré, G. 1998. Velocity macro-model estimation from seismic reflection data by stereotomography $[\hookrightarrow]$. Geophysical Journal International, 135(2), 671-690. 2.3.4, 6.4

Bishop, T.N., Bube, K.P., Cutler, R.T., Langan, R.T., Love, P.L., Resnick, J.R., Shuey, R.T., Spindler, D.A., \& Wyld, H.W. 1985. Tomographic determination of velocity and depth in laterally varying media $[\hookrightarrow]$. Geophysics, 50(6), 903-923. $2.3 .4,6.1,6.4$

Bleistein, N. 1984. Mathematical methods for wave phenomena. Computer Science and Applied Mathematics, vol. 13. New York: Academic press. 2.1.2

Bleistein, N., \& Handelsman, R. 1975. Asymptotic expansions of integrals. New York: Dover. 4.2

Bleistein, N., Cohen, J.K., \& jr., J.W. Stockwell. 2001. Mathematics of multidimensional seismic imaging and inversion. Interdisciplinary applied mathematics, vol. 13. New York: Springer-Verlag. 2.1.2, 2.3.2

Brown, M.P., \& Guitton, A. 2005. Least-squares joint imaging of multiples and primaries $[\hookrightarrow]$. Geophysics, $\mathbf{7 0}(5)$, S79-S89. 3.1

Bunks, C., Saleck, F.M., Zaleski, S., \& Chavent, G. 1995. Multiscale seismic waveform inversion $[\hookrightarrow]$. Geophysics, 60(5), 1457-1473. 2.3.1

Cara, M., \& Leveque, J.J. 1987. Waveform inversion using secondary observables $[\hookrightarrow]$. Geophysical Research Letters, 14(10), 1046-1049. 2.3.5, 5.1, 5.3, 6.4.2 
Chauris, H., \& Noble, M.S. 2001. Two-dimensional velocity macro model estimation from seismic reflection data by local differential semblance optimization: applications to synthetic and real data sets $[\hookrightarrow]$. Geophysical Journal International, 144(1), 14-26. 2.3.3, 4.1, 4.5.2, 4.5.2, 4.9

Chavent, G., \& Clément, F. 1993. Waveform inversion through MBTT formulation $[\hookrightarrow]$. Tech. rept. 1893. INRIA. 3.1

Chavent, G., Clément, F., \& Gómez, S. 1994. Automatic determination of velocities via migration-based traveltime waveform inversion: A synthetic data example $[\hookrightarrow]$. SEG Expanded Abstracts, 13, 1179-1182. 4.1

Claerbout, J.F. 1984. Imaging the earth's interior. Blackwell Scientific Publishers. $2.2,4.1$

Clément, F., Chavent, G., \& Gómez, S. 2001. Migration-based traveltime waveform inversion of 2-D simple structures: A synthetic example $[\hookrightarrow]$. Geophysics, 66(3), 845-860. 2.3.4, 3.1, 4.6.1, 6.1, 6.3

Crase, E., Pica, A., Noble, M., McDonald, J., \& Tarantola, A. 1990. Robust nonlinear waveform inversion: Application to real data $[\hookrightarrow]$. Geophysics, 55, 527-538. 2.3.1

Dahlen, F.A., Hung, S.-H., \& Nolet, G. 2000. Fréchet kernels for finite frequency traveltimes - i. theory $[\hookrightarrow]$. Geophysical Journal International, 141, 157-174. 2.3 .5

de Hoop, M.V., \& Uhlmann, G. 2005. Characterization and 'source-receiver' continuation of seismic reflection data $[\hookrightarrow]$. Communications on Mathematical Physics. 2.3 .4

de Hoop, M.V., \& van der Hilst, R.D. 2005. On sensitivity kernels for 'waveequation' transmission tomography $[\hookrightarrow]$. Geophysical Journal International, 160(3), 621-633. 2.3.5, 5.1, 5.3, 5.3.1, 5.3.2, 5.4, 5.4, 6.4, 6.4.2

de Hoop, M.V., van der Hilst, R.D., \& Shen, P. 2006. Wave-equation reflection tomography: annihilators and sensitivity kernels $[\hookrightarrow]$. Geophysical Journal International, 167, 1332-1352. 2.3.3, 4.1, 4.5.3, 6.1, 6.5

de Hoop, M.V., Smith, H., Uhlmann, G., \& van der Hilst, R.D. 2009. Seismic imaging with the generalized Radon transform: a curvelet transform perspective $[\hookrightarrow]$. Inverse Problems, 25(2), 025005 (21pp). 2.3.2

Dix, C. Hewitt. 1955. Seismic velocities from surface measurements $[\hookrightarrow]$. Geophysics, 20(1), 68-86. 2.1.2, 4.3.1

Doherty, S.M., \& Claerbout, J.F. 1974. Velocity analysis based on the wave equation. $[\hookrightarrow]$. Tech. rept. 1. Stanford Exploration Project. 2.3.3, 3.1, 3.3, 6.1, 6.4 
Duchkov, A.A., \& de Hoop, M.V. 2009. Velocity continuation in the downward continuation approach to seismic imaging $[\hookrightarrow]$. Geophysical Journal International, 176(3), 909-924. 2.3.3

Duchkov, A.A., de Hoop, M.V., \& Barreto, A.S. 2008. Evolution-equation approach to seismic image, and data, continuation $[\hookrightarrow]$. Wave Motion, 45(7-8), 952-969. 2.3 .3

Dussaud, E.A., \& Symes, W.W. 2005. Velocity analysis from interferometric data $[\hookrightarrow]$. SEG Expanded Abstracts, 2237-2241. 3.1

Etgen, J., Gray, S. H., \& Zhang, Y. 2009. An overview of depth imaging in exploration geophysics $[\hookrightarrow]$. Geophysics, 74(6), WCA5-WCA17. 2.3.2

Faye, J.-P., \& Jeannot, J.-P. 1986. Prestack migration velocities from focusing depth analysis $[\hookrightarrow]$. Seg Expanded Abstracts, 5, 438-440. 4.5.3

Folland, G.B. 1989. Harmonic analysis in phase space. Annals of Mathematics Studies. Princeton University Press. 5.3.2

Gaskill, J.D. 1978. Linear systems, Fourier transforms, and optics. John Wiley \& Sons. 2.2.2

Gee, L.S., \& Jordan, T.H. 1992. Generalized seismological data functionals $[\hookrightarrow]$. Geophysical Journal International, 111(2), 363-390. 5.3.2, 6.4.2

Golub, G., \& Pereyra, V. 1973. The differentiation of pseudo-inverses and nonlinear least squares problems whose variables separate $[\hookrightarrow]$. SIAM Journal on Numerical Analysis, 10(2), 413-432. 2.3.4, 4.1, 4.6, 6.3

Guitton, A., \& Symes, W.W. 2003. Robust inversion of seismic data using the huber norm $[\hookrightarrow]$. Geophysics, 68, 1310-1319. 2.3.1

Higginbotham, Joseph H., Brown, Morgan P., \& Clapp, Robert G. 2008. Wave equation migration velocity focusing analysis $[\hookrightarrow]$. SEG Expanded Abstracts, 27, 3083-3087. 4.5.3

Hörmann, G., \& de Hoop, M. V. 2002. Detection of wave front set perturbations via correlation: Foundation for wave-equation tomography $[\hookrightarrow]$. Applicable Analysis, 81, 1443-1465. 5.1, 5.3.1, 5.3.2, 5.3.2, 5.9, 6.4.2

Jannane, M., Beydoun, W., Crase, E., Cao, D., Koren, Z., Landa, E., Mendes, M., Pica, A., Noble, M., Roeth, G., Singh, S., Snieder, R., Tarantola, A., Trezeguet, D., \& Xie, M. 1989. Wavelengths of earth structures that can be resolved from seismic reflection data $[\hookrightarrow]$. Geophysics, 54(7), 906-910. 2.2

Jiang, Z., Sheng, J., Yu, J., Schuster, G. T., \& Hornby, B. E. 2007. Migration methods for imaging different-order multiples $[\hookrightarrow]$. Geophysical Prospecting, 51, $1-19.3 .1$ 
Kanasewich, E.R. 1985. Time sequence analysis in geophysics. University of Alberta Press. 2.1, 4.5.1

Kuvshinov, B.N., \& Mulder, W.A. 2006. The exact solution of the time-harmonic wave equation for a linear velocity profile $[\hookrightarrow]$. Geophysical Journal International, $\mathbf{1 6 7}(2), 659-662.5 .5$

Lailly, P. 1983. The seismic inverse problem as a sequence of before stack migrations[ $\hookrightarrow$. Proc. Conf. Inverse Scattering, Theory and Applications. 2.3.1, 3.1

Li, J., \& Symes, W.W. 2007. Interval velocity estimation via NMO-based differential semblance $[\hookrightarrow]$. Geophysics, 72(6), U75-U88. 2.3.3, 3.1, 4.5.2, 4.5.2

Luo, Y., \& Schuster, G. T. 1991. Wave-equation traveltime inversion $[\hookrightarrow]$. Geophysics, 56(5), 645-653. 2.3.5, 3.2, 4.1, 5.1, 5.3, 5.3.1, 5.10, 6.4.2

MacKay, S., \& Abma, R. 1992. Imaging and velocity analysis with depth-focusing analysis $[\hookrightarrow]$. Geophysics, 57(12), 1608-1622. 3.1, 3.3, 4.5.3

Marquering, H., Nolet, G., \& Dahlen, F.A. 1999. Three-dimensional sensitivity kernels for finite frequency traveltimes: the banana-doughnut paradox $[\hookrightarrow]$. Geophysical Journal International, 132(3), 521-534. 5.4

Mehta, K., Bakulin, A., Kiyashchenko, D., \& Lopez, J. 2008. Comparing virtual versus real crosswell surveys $[\hookrightarrow]$. SEG Expanded Abstracts, 27, 1372-1376. 5.6

Mora, P. 1988. Elastic wave-field inversion of reflection and transmission data $[\hookrightarrow]$. Geophysics, 53(6), 750-759. 3.1

Mulder, W.A., \& Plessix, R.-E. 2002. Time- versus frequency-domain modelling of seismic wave propagation $[\hookrightarrow]$. EAGE Expanded Abstracts, E015-E015. 4.4, 5.6

Mulder, W.A., \& Plessix, R.-E. 2004. A comparison between one-way and two-way wave-equation migration[ $\hookrightarrow$. Geophysics, 69(6), 1491-1504. 2.3.2

Mulder, W.A., \& ten Kroode, A.P.E. 2002. Automatic velocity analysis by differential semblance optimization[ $\hookrightarrow$. Geophysics, 67(4), 1184-1191. 2.3.3, 3.1, 4.1, $4.5 .2,4.5 .2,4.9$

Mulder, W.A., \& van Leeuwen, T. 2008. Automatic migration velocity analysis and multiples $[\hookrightarrow]$. SEG Expanded Abstracts, 27(1), 3128-3132. 6.1

Pekeris, C.L. 1946. Theory of propagation of sound in a half-space of variable sound velocity under conditions of the formation of a shadow zone $[\hookrightarrow]$. Journal of the Acoustical Society of America, 18, 295-315. 5.5

Plessix, R.-E. 2006. A review of the adjoint-state method for computing the gradient of a functional with geophysical applications $[\hookrightarrow]$. Geophysical Journal International, 167(2), 495-503. 2.3.1, 3.8, 4.8, 5.4, 5.6 
Plessix, R.-E., \& Mulder, W. A. 2004. Frequency-domain finite-difference amplitude-preserving migration $[\hookrightarrow]$. Geophysical Journal International, 157, 975-987. 2.3.2

Plessix, R.-E., Chavent, G., \& Roeck, Y.-H. De. 1999. Waveform inversion of reflection seismic data for kinematic parameters by local inversion $[\hookrightarrow] . S I A M$ Journal of Scientific Computing, 20(3), 1033-1052. 3.1

Pratt, R.G., \& Hicks, G.J. 1998. Gauss-Newton and full-Newton methods in frequency space seismic waveform inversion $[\hookrightarrow]$. Geophysical Journal International, 133(2), 341-362. 2.3.1, 3.1

Pratt, R.G., Song, Z.M., Williamson, P., \& Warner, M. 1996. Two-dimensional velocity models from wide-angle seismic data by wavefield inversion $[\hookrightarrow] . \quad$ Geophysical Journal International, 124(2), 232-340. 3.1

Rickett, J.E., \& Sava, P.C. 2002. Offset and angle-domain common image-point gathers for shot-profile migration $[\hookrightarrow]$. Geophysics, 67(3), 883-889. 2.3.3, 3.1, 3.3, 4.5 .3

Santosa, F., \& Symes, W.W. 1989. An analysis of least-squares velocity inversion. Geophysical monograph series, vol. 4. Society of Exploration Geophysicists. 6.3

Sava, P.C., \& Biondi, B. 2004. Wave-equation migration velocity analysis. I. Theory $[\hookrightarrow]$. Geophysical Prospecting, 52, 593-606. 3.1, 3.3, 6.1, 6.4

Sava, P.C., \& Fomel, S. 2006. Time-shift imaging condition in seismic migration $[\hookrightarrow]$. Geophysics, 71(6), S209-S217. 2.3.3, 2.3.3, 3.1, 3.3, 4.5.3

Shen, P., \& Symes, W.W. 2008. Automatic velocity analysis via shot profile migration $[\hookrightarrow]$. Geophysics, 73(5), VE49-VE59. 2.3.3, 4.1, 4.5.3, 4.9, 6.1, 6.4

Shen, P., Symes, W. W., \& Stolk, C. C. 2003. Differential semblance velocity analysis by wave-equation migration $[\hookrightarrow]$. SEG Expanded Abstracts, 2132-2135. $2.3 .3,3.1,3.2,3.3$

Sheriff, R.E., \& Geldart, L.P. 1982. Exploration seismology. Cambridge university press. 2.1

Shin, C., \& Cha, Y.H. 2008. Waveform inversion in the Laplace domain $[\hookrightarrow]$. Geophysical Journal International, 132, 922-931. 2.3.1

Shin, C., \& Cha, Y.H. 2009. Waveform inversion in the Laplace-Fourier domain $[\hookrightarrow]$. Geophysical Journal International, 177, 1067-1079. 2.3.1

Shin, C., \& Min, D.-J. 2006. Waveform inversion using a logarithmic wavefield $[\hookrightarrow]$. Geophysics, 71(3), R31-R42. 2.3.1

Stolk, C.C., \& de Hoop, M.V. 2006. Seismic inverse scattering in the downward continuation approach $[\hookrightarrow]$. Wave Motion, 43, 579-598. 2.3.2, 2.3.3, 4.1, 4.5.3, 5.3 .2 
Stolk, C.C., \& Symes, W.W. 2003. Smooth objective functionals for seismic velocity inversion $[\hookrightarrow]$. Inverse Problems, 19, 73-89. 2.3.3, 2.3.4, 4.1, 4.6.1, 6.3

Stolk, C.C., \& Symes, W.W. 2004. Kinematic artifacts in prestack depth migra$\operatorname{tion}[\hookrightarrow]$. Geophysics, 69(2), 562575. 2.3.3

Stolk, C.C., de Hoop, M.V., \& Symes, W.W. 2009. Kinematics of shot-geophone migration $[\hookrightarrow]$. Geophysics, 74(6), WCA19-WCA34. 2.3.3

Stork, C. 1992. Reflection tomography in the postmigrated domain $[\hookrightarrow]$. Geophysics, 57(5), 680-692. 3.1

Strichartz, R.S. 2003. A guide to distribution theory and Fourier transforms. World Scientific. 5.3.2

Symes, W.W. 1991. Layered velocity inversion: A model problem from reflection seismology $[\hookrightarrow]$. SIAM Journal on Mathematical Analysis, 22(3), 680-716. 2.2.3, $4.1,4.5 .2$

Symes, W.W. 1999. All stationary points of differential are global minimizers: Layered acoustics $[\hookrightarrow]$. Tech. rept. 100. Stanford Exploration Project. 2.3.3, 3.4, 3.8, $4.3 .1,4.5 .2$

Symes, W.W. 2008a. Approximate linearized inversion by optimal scaling of prestack depth migration[ $\hookrightarrow$. Geophysics, 73(2), R23-R35. 2.3.3, 4.1, 6.1, 6.6

Symes, W.W. 2008b. Migration velocity analysis and waveform inversion $[\hookrightarrow]$. Geophysical Prospecting, 56(6), 765-790. 2.3.3, 4.6.1, 5.3.2, 7

Symes, W.W., \& Carazzone, J.J. 1991. Velocity inversion by differential semblance optimization $[\hookrightarrow]$. Geophysics, 56(5), 654-663. 2.3.3, 3.1, 3.3, 6.4

Tarantola, A. 1984. Inversion of seismic reflection data in the acoustic approxima$\operatorname{tion}[\hookrightarrow]$. Geophysics, 49(8), 1259-1266. 3.1, 4.1, 5.4

Tarantola, A., \& Valette, A. 1982. Generalized nonlinear inverse problems solved using the least squares criterion $[\hookrightarrow]$. Reviews of Geophysics and Space Physics, 20(2), 129-232. 2.3.1, 3.1, 4.6, 5.1, 6.3

ten Kroode, A.P.E., Smit, D.-J., \& Verdel, A.R. 1998. A mircrolocal analysis of migration $[\hookrightarrow]$. Wave Motion, 28, 149-172. 2.3.2

Toldi, J.L. 1989. Velocity analysis without picking $[\hookrightarrow]$. Geophysics, 54(2), 191-199. 2.3.3, 4.5 .1

Tromp, J., Tape, C., \& Liu, Q. 2005. Seismic tomography, adjoint methods, time reversel and banana-doughnut kernels $[\hookrightarrow]$. Geophysical Journal International, 160, 195-216. 5.1, 5.6

van Leeuwen, T., \& Mulder, W.A. 2008a. Velocity analysis based on data correlation $[\hookrightarrow]$. Geophysical Prospecting, 56(6), 791-803. 4.1, 4.6.2, 5.3.2, 6.1, 6.4.2, 6.4 .2 
van Leeuwen, T., \& Mulder, W.A. 2008b. Velocity analysis in the data domain overview and prospects $[\hookrightarrow]$. EAGE Extended Abstracts. 4.6.2

van Leeuwen, T., \& Mulder, W.A. 2008c. Velocity analysis with multiples - NMO modeling for layered velocity structures $[\hookrightarrow]$. SEG Expanded Abstracts, 27(1), 1925-1929. 7.1

van Leeuwen, T., \& Mulder, W.A. 2010a. A comparison of seismic velocity inversion methods for layered acoustics $[\hookrightarrow]$. Inverse Problems, 26(1), 015008 (21pp). 5.3.2, $6.4 .2,6.4 .2,6.6 .1$

van Leeuwen, T., \& Mulder, W.A. 2010b. A correlation-based misfit criterion for wave-equation traveltime tomography $[\hookrightarrow]$. Geophysical Journal International (in print, published online). 6.1, 6.4.2, 6.4.2, 7

Červený, V. 2001. Seismic ray theory. Cambridge University Press. 2.1.2, 2.1.2

Versteeg, R.J. 1993. Sensitivity of prestack depth migration to the velocity $\operatorname{model}[\hookrightarrow]$. Geophysics, 58(6), 873-882. 4.8

Virieux, J., \& Operto, S. 2009. An overview of full-waveform inversion in exploration geophysics $[\hookrightarrow]$. Geophysics, 74(6), WCC1-WCC26. 2.3.1

Vogel, C.R. 2002. Computational methods for inverse problems. Frontiers in Applied Mathematics, vol. 23. Philadelphia: SIAM. 1.2, 3.5, 5.1

Wang, Y., \& Pratt, R.G. 1997. Sensitivities of seismic traveltimes and amplitudes in reflection tomography[ $[$. Geophysical Journal International, 131(3), 618-642. 2.2

Wapenaar, C.P.A, \& Berkhout, A.J. 1989. Elastic wave field extrapolation. Advances in exploration geophysics. Amsterdam: Elsevier. 2.1

Yilmaz, O. 1987. Seismic data processing. Atlantic Books. 4.1

Yilmaz, O., \& Chambers, R. 1984. Migration velocity analysis by wavefield extrapolation $[\hookrightarrow]$. Geophysics, 1664-1674. 2.3.2, 3.1, 3.3

Youn, O.K., \& Zhou, H. 2001. Depth imaging with multiples $[\hookrightarrow]$. Geophysics, 66(1), 246-255. 3.1 


\section{Summary}

\section{Correlation-based seismic velocity inversion}

Most of our knowledge of the subsurface comes from the measurement of quantities that are indirectly related to the earth's structure. Examples are seismic waves, gravity and electromagnetic waves. We consider the use of seismic waves for inference of structural information on an exploration scale. The seismic waves are generated by placing sources at or near the surface and the response is measured with either geophones or hydrophones. From such data, valuable information on the earth's structure may be inferred up to roughly $10 \mathrm{~km}$ depth with a resolution of 50 - $100 \mathrm{~m}$. While such data where interpreted directly in the early days, present-day processing involve detailed mathematical description of wave propagation in terms of density and sound speeds. With the aid of such a mathematical description, the actual seismic experiment may be simulated on a computer. The parameters (density, sound speed) that best describe the subsurface can then be obtained by trying to match the observed data to the simulated data. Such a problem is called an inverse problem. A straightforward way to solve this is to try to fit the data in a least-squares sense. However, due to the specifics of the seismic experiment this only works well for parameters that govern the dynamics of the data. The kinematics are, however, also very important as these are sensitive to large scale velocity changes. To successfully invert the kinematics of the data, the inverse problem needs to be reformulated. In this thesis we investigate such a reformulation. The basic ingredient of this reformulation is the way the mismatch between the data are measured. Instead of subtracting the data - as in the least-squares approach - we infer the kinematic difference (the timeshift, basically) between the simulated and observed data via a weighted norm of the correlation. We discuss the application of this basic principle to both reflection and transmission data. We find that the weighted norm of the correlation is a robust way to measure the shift between complex waveforms and can be successfully applied to cross-well tomography and velocity inversion for horizontally layered media. Preliminary results for non-layered media suggest that the approach is applicable in this case as well.

- Tristan van Leeuwen. 


\section{Samenvatting}

\section{Inversie van seismische voortplantingsnelheid gebaseerd op corre- latie}

Een groot deel van onze kennis van de ondergrond wordt verkregen via het meten van grootheden die zich indirect verhouden tot de fysische parameters in de aarde. Voorbeelden zijn seismische golven, zwaartekracht en elektromagnetische golven. Wij beschouwen het gebruik van seismische golven om een structureel beeld van de ondergrond te verkrijgen op exploratie schaal. De seismische golven worden opgewekt door middel van seismische bronnen welke aan het oppervlak worden geplaatst en waarvan de respons wordt gemeten met geo- of hydrofoons. Uit zulke metingen kan waardevolle informatie over de ondergrondse structuur worden verkregen tot op een diepte van ongeveer $10 \mathrm{~km}$ met een resolutie van grofweg $50-100 \mathrm{~m}$. Vroeger werden zulke metingen direct geïnterpreteerd, maar tegenwoordig gebruikt men diverse nauwkeurige wiskundige beschrijvingen van golfvoortplanting in termen van relevante fysische parameters zoals voortplantingssnelheid en dichtheid. Met behulp van zo een wiskundige beschrijving kan het daadwerkelijke seismische experiment worden nagebootst op een computer. De fysische parameters die de aarde het best beschrijven kunnen dan worden afgeleid door de nagebootste metingen zo goed mogelijk op de echte metingen te laten lijken. Zo'n probleem heet een inverse probleem. Een gebruikelijke manier om zo'n probleem aan te pakken is om het kleinste kwadratenverschil tussen de metingen en de nagebootste metingen te minimaliseren. Echter, het blijkt dat deze aanpak alleen goed werkt voor fysische parameters die de amplitudes van de gereflecteerde golven bepalen. De reistijden van de golven zijn ook erg belangrijk, omdat deze gevoeliger zijn voor grootschalige veranderingen in de voortplantingssnelheid. Om de reistijden optimaal te kunnen gebruiken moeten we het inverse probleem anders formuleren. In dit proefschrift onderzoeken we zo'n alternatieve formulering. Het basisingrediënt is de manier waarop het verschil tussen de nagebootste en daadwerkelijke metingen wordt bepaald. In plaats van de metingen van elkaar af te trekken - zoals met de kleinste kwadratenaanpak - gebruiken we kruiscorrelatie om de verschuiving tussen de metingen te bepalen via een gewogen norm van de correlatie. We bespreken het gebruik van dit basisprincipe in verschillende toepassingen. We concluderen dat de gewogen norm van de correlatie een robuuste manier is om het kinematische verschil tussen twee golfvormen te meten. We laten ook zien dat het principe succesvol kan worden toegepast op 'cross-well' tomografie en snelheidsanalyse voor horizontaal gelaagde media. Eerste resultaten voor niet-gelaagde media suggereren dat toepassing ook in dit meer algemene geval mogelijk is.

- Tristan van Leeuwen. 


\section{Curriculum Vitae}

Tristan van Leeuwen was born on the 26th of November 1981 in Hilversum, where he got his VWO diploma in 2000. He started his studies in Computational Science at the Mathematics department of Utrecht University, during which he did an internship at the RIZA in Arnhem, developing a numerical method for the study of sediment flow in rivers. He obtained his Masters degree in Computational Science in 2006 for work on parallel computing under the supervision of Prof.dr. R. Bisseling. Immediately following the completion of his M.Sc. Tristan joined the Applied Geophysics group at the department of Civil Engineering and Geotechnology at the Technical University of Delft. Under the supervision of Prof.dr. W.A. Mulder he worked on seismic velocity inversion. During this period Tristan visited Prof.dr. W.W. Symes at Rice University in the Spring of 2007 for three months and in the Spring of 2009 for two weeks as well as Prof.dr. M.V. de Hoop at Purdue University for two weeks in the Spring of 2009. His research led to three journal papers and several conference abstracts. In 2009 Tristan received the Society of Exploration Geophysicists best student paper award for his contribution to the annual conference in 2008.

In September he and his girlfriend are moving to Vancouver where Tristan will join Prof.dr. F.J. Hermann's group at the University of British Columbia. 


\section{Acknowledgements}

This work is part of the research programme of the Foundation for Fundamental Research on Matter (FOM), which is financially supported by the Netherlands Organisation for Scientific Research (NWO). We acknowledge the NAM ('Nederlands Aardolie Maatschappij') for providing the data used in chapter 3 and Shell Oil for providing the cross-well data used in chapter 5.

I would personally like to thank everybody who contributed - directly or indirectly - to this thesis. Family, friends, colleagues, pets: thank you. 
Present-day oil exploration relies heavily on advanced seismic imaging algorithms. The main goal of such algorithms is to map the recorded data, as a function of time and source/receiver position, to an image of the subsurface, as a function of lateral position and depth. The main prerequisite is knowledge of the velocity with which the waves have travelled in the subsurface. While modern imaging algorithms take into account finite frequency effects of wave propagation and are fully automated, most methods to estimate the subsurface velocity are still based on a geometric optics approximation to wave propagation and require a lot of manual intervention. Therefore, considerable effort is being put into developing a fully automated method to estimate the subsurface velocities using finite-frequency, two-way, wave propagation. A promising line of research that has been developed over the last few years poses velocity estimation as a PDE-constrained optimization problem. This thesis describes one possible way to formulate such an optimization problem. The main ingredient of this novel formulation is a correlation-based method to measure the kinematic difference between wave fields. The thesis consists of an introductory section which briefly describes the velocity estimation problem and reviews the literature, three previously published papers which describe the use of the correlation-based method for laterally invariant media and transmission tomography, and a chapter with preliminary results on laterally varying media.

ISBN 978-90-8891-186-6

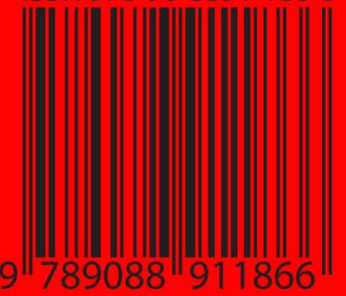

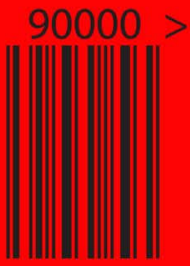

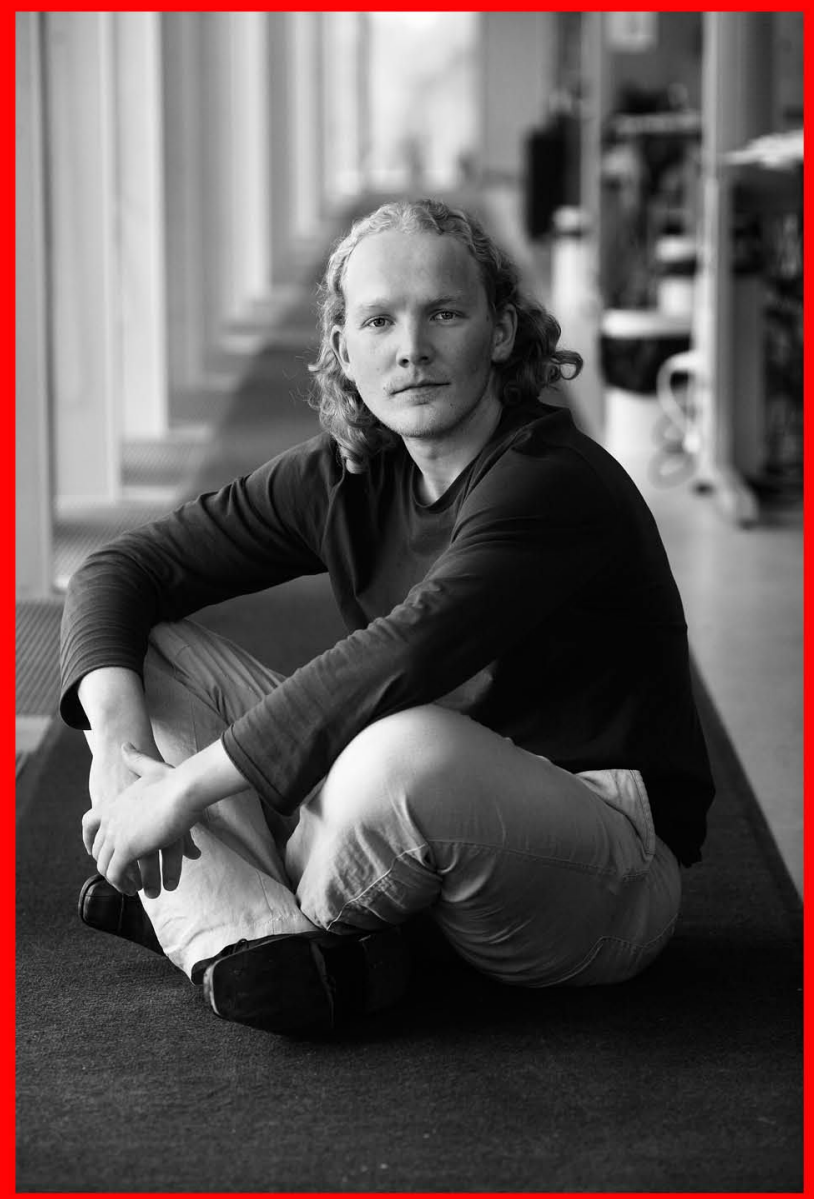

